DOE/ER/40443--1

DE93 010765

\title{
STUDIES OF NUCLEI USING RADIOACTIVE BEAMS
}

Progress Report

May 1988 - July 1989

Dr. Rodney B. Piercey

University of Florida

Space Astronomy Laboratory

1810 N.W. 6th Street

Gainesville, FL. 32609

July 1989

Prepared For the United States Department Of Energy

Under Grant Number DE-FG05-88ER40443 


\begin{abstract}
The 12 month period from May 1988 to July 1989 represents the first full year of our 18 month pilot program in nuclear structure research. In this period, research was initiated to develop a capability for radioactive secondary beams at Argonne National Laboratory using the Atlas and the new Fragment Mass Analyzer (FMA) which is currently under construction. Two major new detector facilities are currently in the final stages of design and testing. The Large-Area, Scintillator Telescope (LAST) detector is fully operational and will be shipped to Argonne National Laboratory in August for fit-tests and in-beam calibrations. The first segments of a new sixteen-segment neutron multiplicity detector detector have been built and tested. The remaining segments are currently being constructed. Research was continued in the areas of: (1) Coulomb excitation studies of rare earth and actinide nuclei; (2) In-beam, gamma-ray spectroscopy of nuclei in the mass 100 region, and (3) Advanced detector design. Several journal articles and abstracts were published or submitted for publication in the reporting period and others are currently in preparation. Three graduate students participated in the program. One from the University of Florida and two from the Royal Institute of Technology, Stockholm, Sweden.
\end{abstract}

\title{
DISCLAIMER
}

This report was prepared as an account of work sponsored by an agency of the United States Government. Neither the United States Government nor any agency thereof, nor any of their employees, makes any warranty, express or implied, or assumes any legal liability or responsibility for the accuracy, completeness, or usefulness of any information, apparatus, product, or process disclosed, or represents that its use would not infringe privately owned rights. Reference herein to any specific commercial product, process, or service by trade name, trademark, manufacturer, or otherwise does not necessarily constitute or imply its enjorsement, recommendation, or favoring by the United States Government or any agency thereof. The views and opinions of authurs expressed herein do not necessarily state or reflect those of the United States Government or any agency thereof. 


\section{CONTENTS}

\section{ABSTRACT \\ INTRODUCTION}

SECTION-I EXPERIMENTAL ACTIVITIES

1.0 - Large-Area, Scintillation Telescone (LAST) Detector Development

2.0 - A New 16-Segment Neutron Detector

3.0 - High spin states in ${ }^{72} \mathrm{Ge}$

1.0 - "Coulomb Excitation of 144,146,148,150 Nd" 16

2.0 - "Columb-Nuclear Interference Measurements of the Hexadecapole 21

Deformation in ${ }^{168} \mathrm{Yb}$ and $178,180 \mathrm{Hf}^{\prime \prime}$

3.0 - "Preliminary Characteristics of a Germanium-Based, Comptom-Scatter, Gamma-ray Telescope"

4.0 - "Inconsistency of Hexadecapole Matrix Element for ${ }^{180} \mathrm{Hf}$ as Extracted from Nuclear and Leptonic Scattering"

5.0 - "Coulomb Excitation to High Spin in ${ }^{248} \mathrm{Cm} "$ 53

6.0 - "A Germanium-Based, Comptom-Scatter, Gamma-ray Telescope"

7.0 - "A Large-Area, Scintillation Telescope for Heavy Ions (LAST)"

8.0 - "Low Energy Heavy Ion Fragment Identification"

SECTION-III INVITED TALKS, PRESENTATIONS AND OTHER TRAVEL 102

SECTION-IV PERSONNEL 10,3

$\begin{array}{ll}\text { Appendix-A Student Report, Tomas Dybler } & 104\end{array}$

Appendix-B Student Report, Peter Agnvall 134

Appendix-C LAST Detector Engineering Drawings $\quad 158$ 


\section{INTRODUCTION}

This report describes the activities of the experimental nuclear physics group at the University of Florida Space Astronomy Laboratory under contract DE-FG05-88ER40443 with the Department of Energy for the period from May, 1988 to July, 1989. This first year of our pilot program of basic research in experimental nuclear physics has seen significant progress in several areas but has been dedicated primarily to detector development. Subsequent to the approval of our proposal, funding was approved for the new Fragment Mass Analyzer (FMA) at Argonne National Laboratory. The combination of the Atlas accelerator and the FMA offered broader capabilities than the Recoil Mass Separator at Nuclear Structure Research Laboratory (NSRL) where we had originally planned to develop radioactive beam capabilities. Also the FMA schedule corresponded roughly to the anticipated time-line for our program. It was decided, based on these new opportunities to participate in the FMA project at ANL and to concentrate our nuclear structure research with radioactive ion beams (RIBs) at Argonne.

An essential item in the utilization of the FMA for RIBs is the development of appropriate detectors to be used at the primary target and the focal plane for the detection and identification of the secondary ions. In just 12 months we have designed, constructed and tested an ultra-fast focal-plane detector based on large-area thin scintillator foils. Our Large-Area, Scintillation Telescope (LAST) detector in undergoing final in-house tests using a fission source prior to being shipped to Argonne for in-beam tests. The LAST detector was designed, fabricated and tested completely at the University of Florida. Preliminary tests of of the detector concept have already been carried out at Oak Ridge National Laboratory and a preliminary analysis of the data is underway. Abstracts describing both the in-house tests of the full scale detector and the preliminary in-beam tests have been submitted to the 1989 IEEE NSS meeting.

Based on our previous work in designing and fabricating the PANDA neutron multiplicity detector, we have designed a new 16-segment neutron multiplicity detector which will also be used with the FMA. Neutron-gamma discrimination is accomplished by integrating the charge in the PMT output over short and long timescales. A test segment has been constructed and the preliminary tests indicate excellent neutron-gamma discrimination. The independent-segment design will allow enough flexibility to configure the neutron detector array with most other detector-target geometries. The 16 -segment version will also provide 
a much improved multiplicity resolution as compared to the original 5-segment PANDA design.

While the excited states of ${ }^{72} \mathrm{Se}$ have been studies for several years, there still exist many questions about the structure at high spin. At ORNL we have performed in an experiment which used the compact germanium ball to study high spin states in ${ }^{72} \mathrm{Se}$. Data analysis is currently underway.

We have finished the analysis of data taken at several other experiments and have published or submitted for publication articles and abstracts on (1)-Coulomb Excitation of $144,146,148,150 \mathrm{Nd},(2)$-Columb-Nuclear Interference Measurements of the Hexadecapole Deformation in ${ }^{168} \mathrm{Yb}$ and $178,180 \mathrm{Hf}$, (3)-Preliminary Characteristics of a GermaniumBased Compton-Scatter Telescope, (4)-Inconsistency of Hexadecapole Matrix Element for $180 \mathrm{Hf}$ as Extracted from Nuclear and Leptonic Scattering, (5)-Coulomb Excitation to High Spin in ${ }^{248} \mathrm{Cm}$, (6)-A Germanium-Based, Comptom-Scatter, Gamma-ray Telescope, (7)A Large-Area, Scintillation Telescope for Heavy Ions (LAST), and (8)-Low Energy Heavy Ion Fragment Identification.

In other activities we participated in the Workshop on the FMA at Argonne National Laboratory and the pre-town meeting at Argonne to draft input for the NSAC long-range plan and we have continued our guest-student program with the Royal Institute of Sweden. Two students, Peter Agnvahl and Tomas Dybler studied three months each in our group and wrote reports on there activities. 
SECTION-I EXPERIMENTAL ACTIVITIES 


\section{0 - Large-Area, Scintillation Telescope (LAST) Detector Development}

A large part of what we know about nuclear structure has been deduced from the results of nuclear reaction produced by bombarding stable targets with stable beams. Current theories of nuclear structure predict around 8000 species of nuclides to be bound with lifetimes longer than micro-seconds. Of these only about 2000 have been identified to date and less than 300 are among the stable nuclides. Interest in experimental techniques that utilize secondary radioactive ion beams has continued to increase since we initiated our program and a new push for a national RIB facility is gaining strength. Until such a facility is available, RIBs may be produced at low to moderate intensities with existing and planned recoil mass spectrometers located at major accelerator facilities. We have design, fabricated and tested a Large-Area, Scintillation Telescope (LAST) to be used as a fast focal-plane detector on the new Argonne Fragment Mass Analyzer (FMA). The LAST detector is designed to allow the fast $Z$ identification of secondary beams arriving at the focal plane of the FMA and can be used as a trigger to study reactions produced by these beams.

The new detector uses thin foils of plastic scintillator viewed by photomultipliers to detect transiting ions at the focal plane of the FMA. Thin plastic films are known to survive a much higher total radiation dose then solid state detectors (at least two orders of magnitude) and, therefore, are preferable for many applications which require high fluxes at the focal plane. The durability and excellent timing characteristics make this detector particularly valuable for secondary beams measurements. Since the energy loss in ultrathin films can be quite small, the detector can be used when the energy of the transiting iors is low. Furthermore, since these detectors are transition detectors and remove so little energy from the ions, it is practical to use them in conjunction with other detectors at the focal plane to improve crucial measurements such as nuclear $\mathrm{Z}$ determination. The dual-

module thin-film detector for the FMA provides new capabilities which will allow the application of techniques not possible with either solid state or gas counters alone. 
Our design incorporates one or more LAST detector modules, each of which can provide a fast timing trigger and a pulse height related to the energy loss in the film. Accurate velocities can then be determined from the time differences and ion energies can then be calculated from the ion mass. The $\Delta \mathrm{E}$ values can be used alone or in conjunction with other focal plane devices to determine the $\mathrm{Z}$ of the transiting ion. Furthermore, a LAST module can perform without degradation at much higher counting rates and total dose than other detectors designs.

The principle of the LAST detector is illustrated in figure 1. Heavy ions arriving at the focal plane of the FMA will pass through two thin scintillator foils. Each of the foils is viewed by two large photomultipliers which are used to measure the pulse height and time of the transit. This information is then used to determine the velocity from the time-offlight and the energy loss.

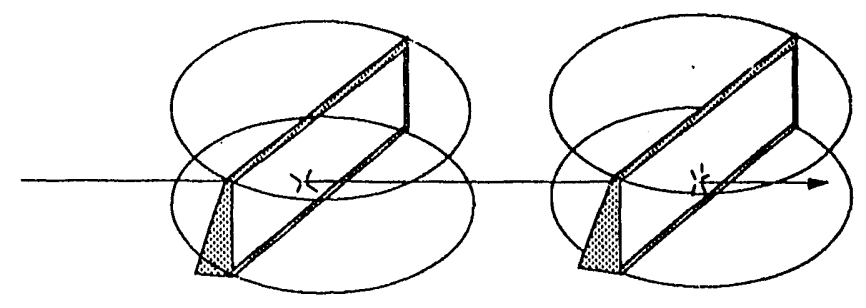

Figure 1. Principle of the LAST Detector

Delta-E transmission detectors must be very thin for use with low-energy heavy-ions and the plastic scintillator foils can be made more than an order of magnitude thinner than solid state detectors. The foils are also very durable and inexpensive to produce.

There is no direct coupling of the foil to the photomultipliers. The PMTs view the foil through quartz windows and rely on there large solid angles for efficient light collection. Due to the light collection geometry the chambers must be light-tight. Ion gauges near the chambers can produce intolerable backgrounds. 
The scintillator foils are housed in cubical chambers with the PMTs mounted on the top and bottom. Figure 2 shows a two chamber arrangement which can be used to measure the energy loss in each foil and the time-of-flight between the foils or between the foils and a pulsed-beam trigger.

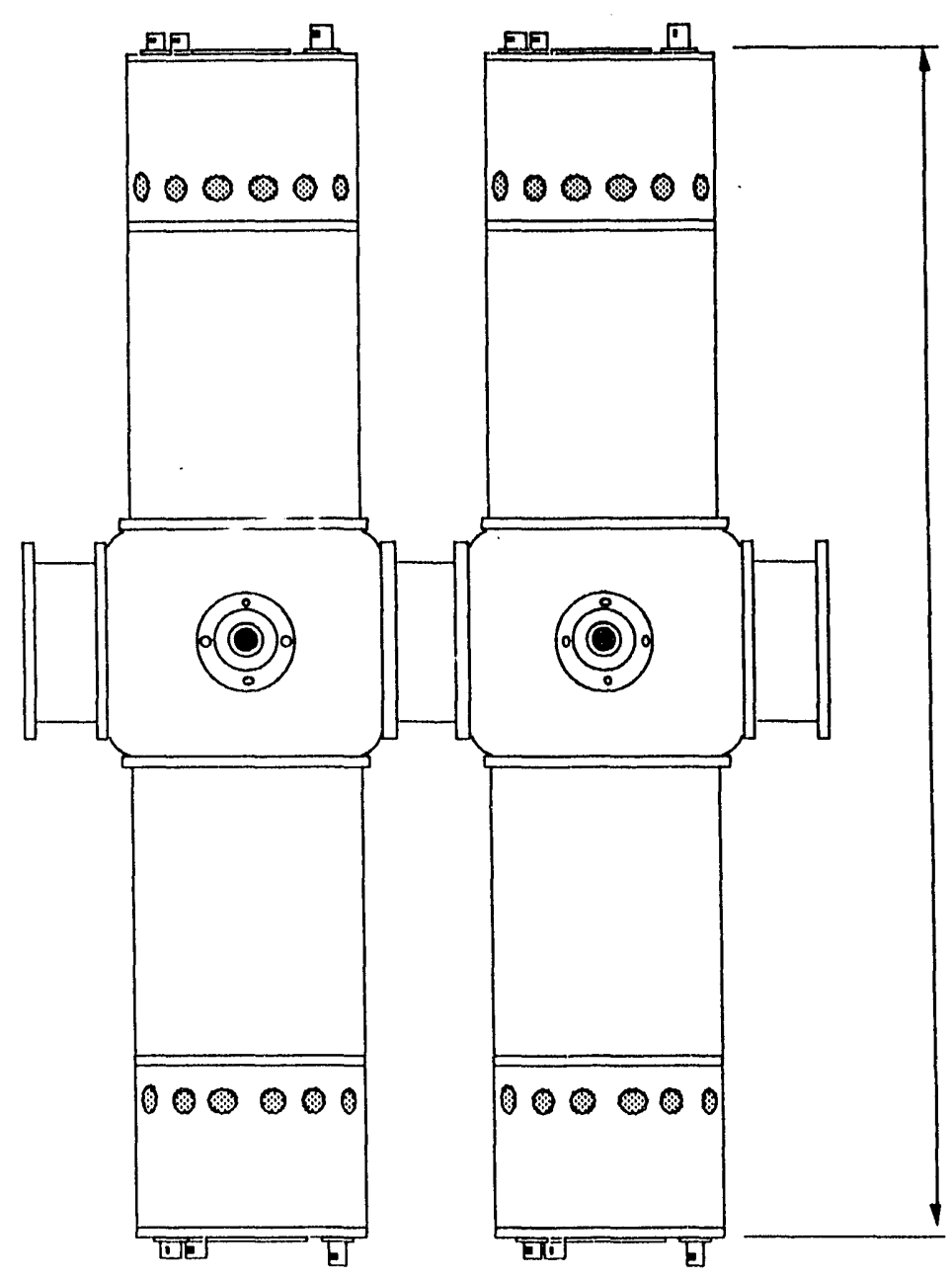

Figure 2. A two chamber LAST configuration.

The foils may be withdrawn or rotated by two rotary-linear feed-throughs mounted on the sides of each chamber. By rotating the foils, the effective thickness may be varied to increase the energy loss and light out-put. A blank-off on the opposite side covers an additional port which can be used to provide additional access for other sensors or electrical freed-through. 
The photomultiplier socket and voltage divider components are mounted on a round circuit board inside the photomultiplier assembly as shown in figure 3. Connections are made to both sides of the base plate to allow for easy removal and maintenance.

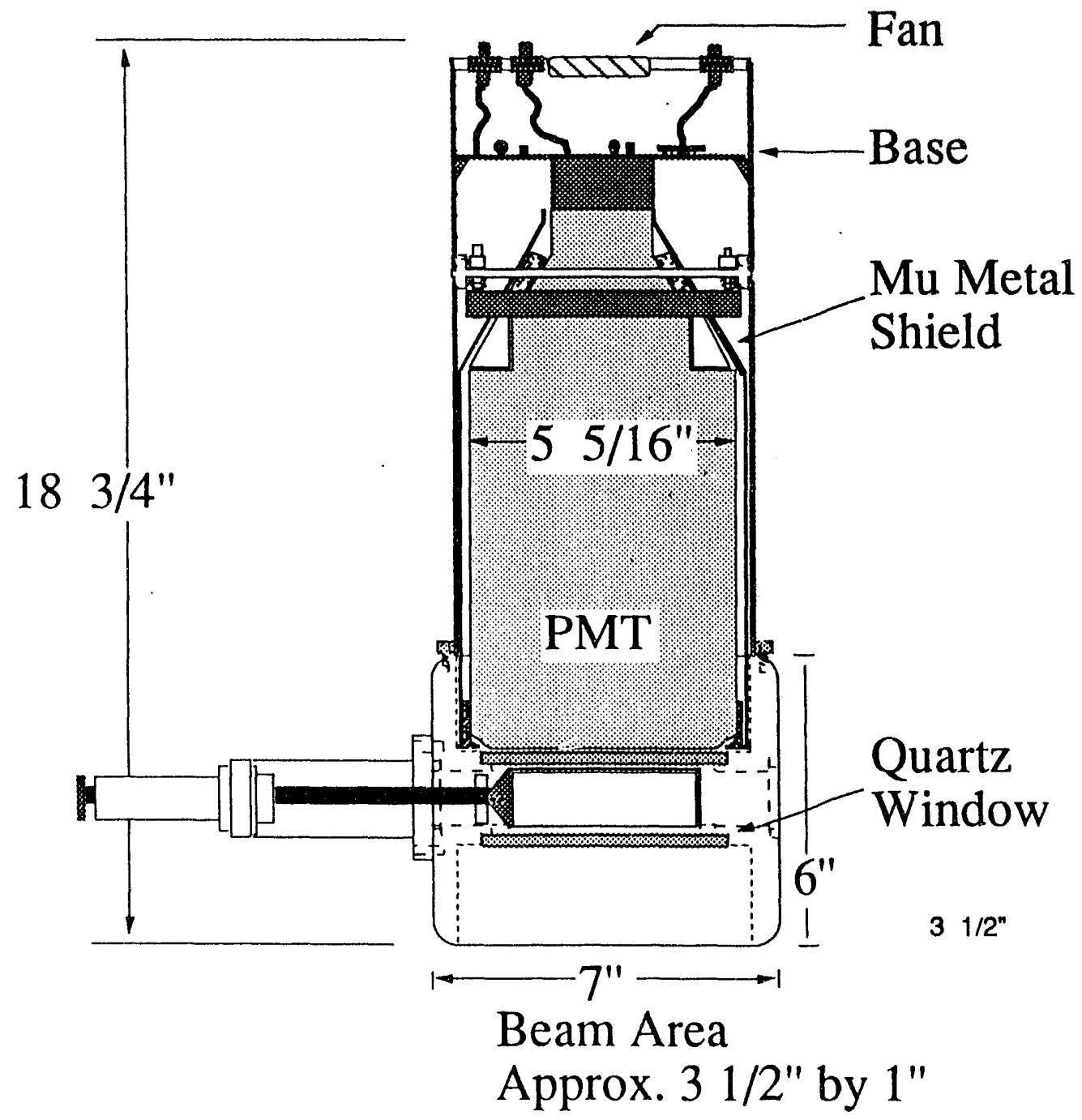

Figure 3. The LAST Detector Mounted at the Focal Plane of the FMA.

The bases normally run at 7 to 8 milliamps to insure stability at high count rates. Fans mounted in the base plates provide cooling for the bases for further stability. Mu metal shields around the tubes isolate the dynode chain from the stray fields produced be the FMA or beam focusing elements. 
Initial tests using a fission source indicate good pulse height resolution and good timing. Figure 4. shows the pulse height spectrum from one of the photomultipliers on one of the chambers. There is an anomalously high noise in this measurement, but the heavy and light mass fragment peaks are both clearly visible.

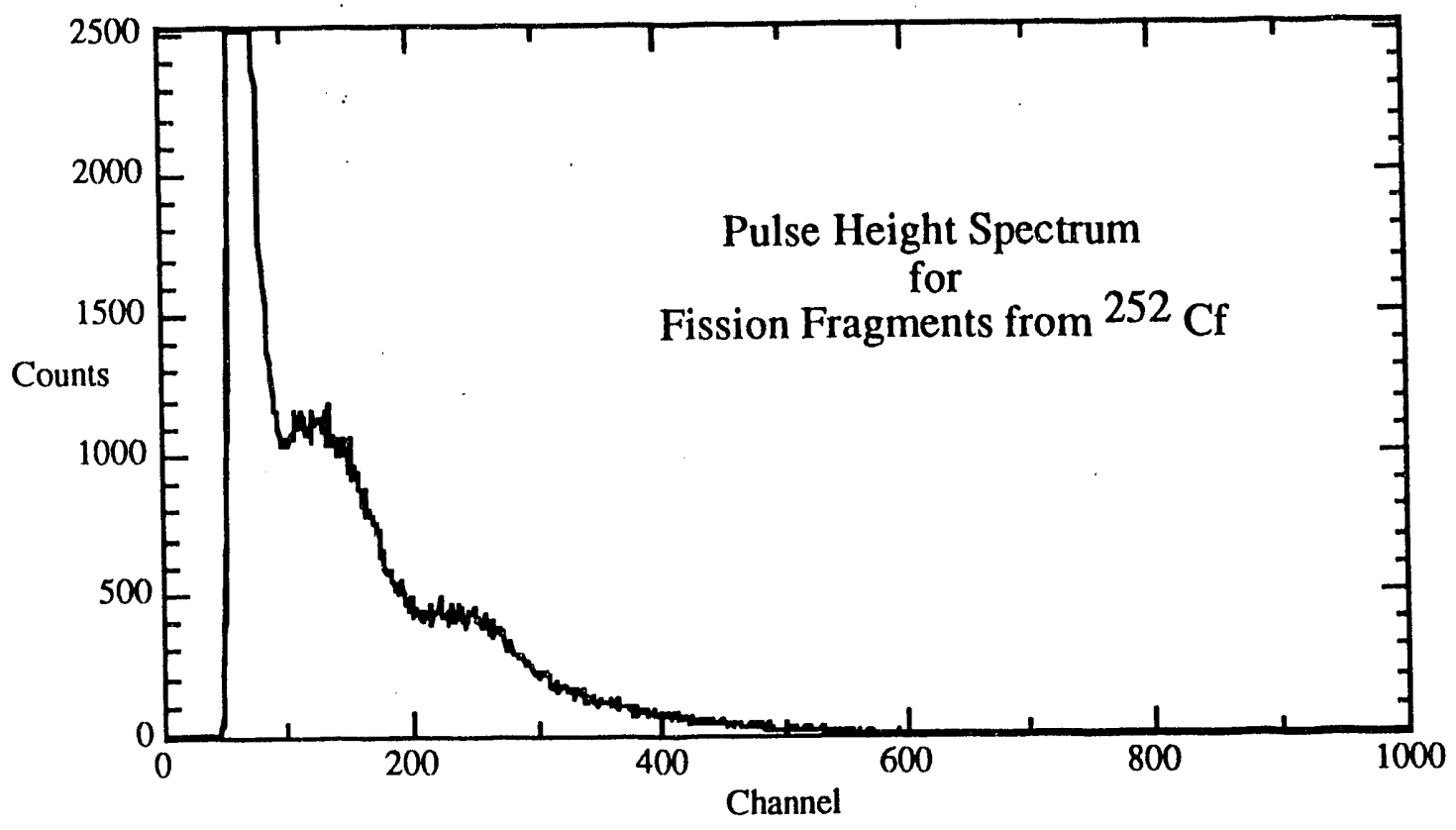

Figure 4. Pulse Height Spectrum From One Photomultiplier. 
Figure 5 shows the time spectrum between two opposing photomultipliers on one of the LAST chambers. These results were achieved as soon as the chamber was assembled and we believe that with some tuning and better CFDs we should be able to achieve substantial improvements.

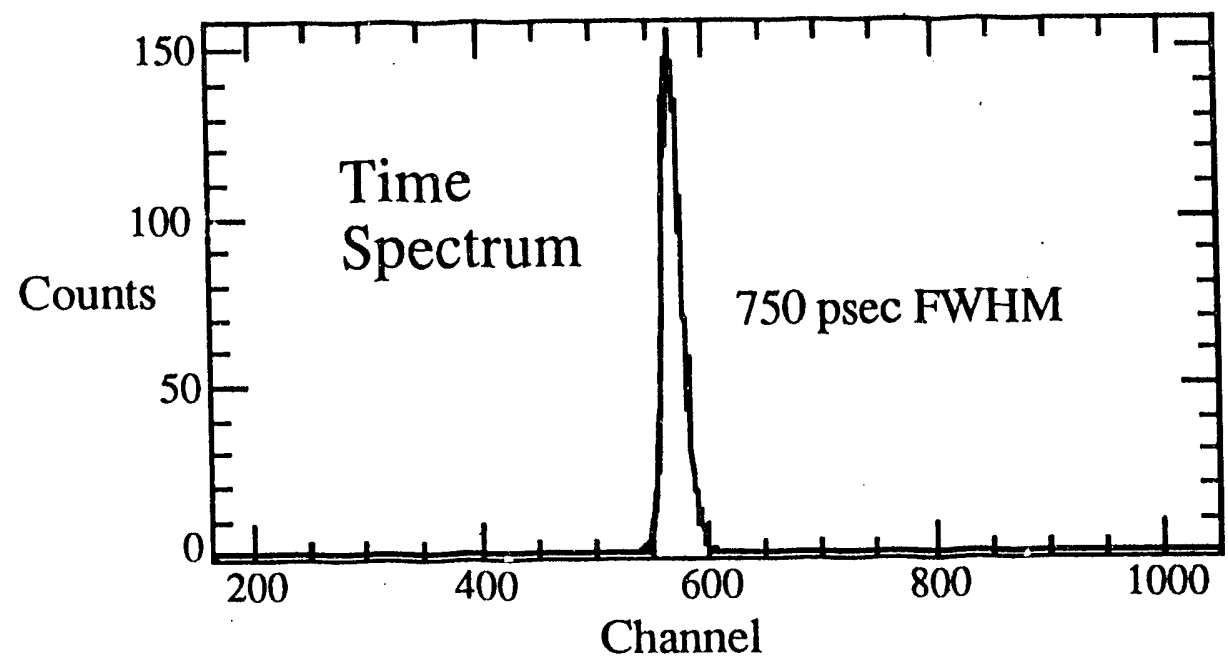

Figure 5. Time Spectrum Between Photomultipliers on the Same Chamber..

Assuming a timing trigger at the primary target and a flight time of 10 microsecs we would be abie to measure the arrival time to better that one part in $10^{4}$. In addition this could be done at high rates and in real time to select specific reaction channels. 
One or both of the LAST detector chambers can be used at in conjunction with other detectors as shown in figure 5. This allows for a highly flexible system and suggest several applications in addition to it use in identifying secondary radioactive beams.

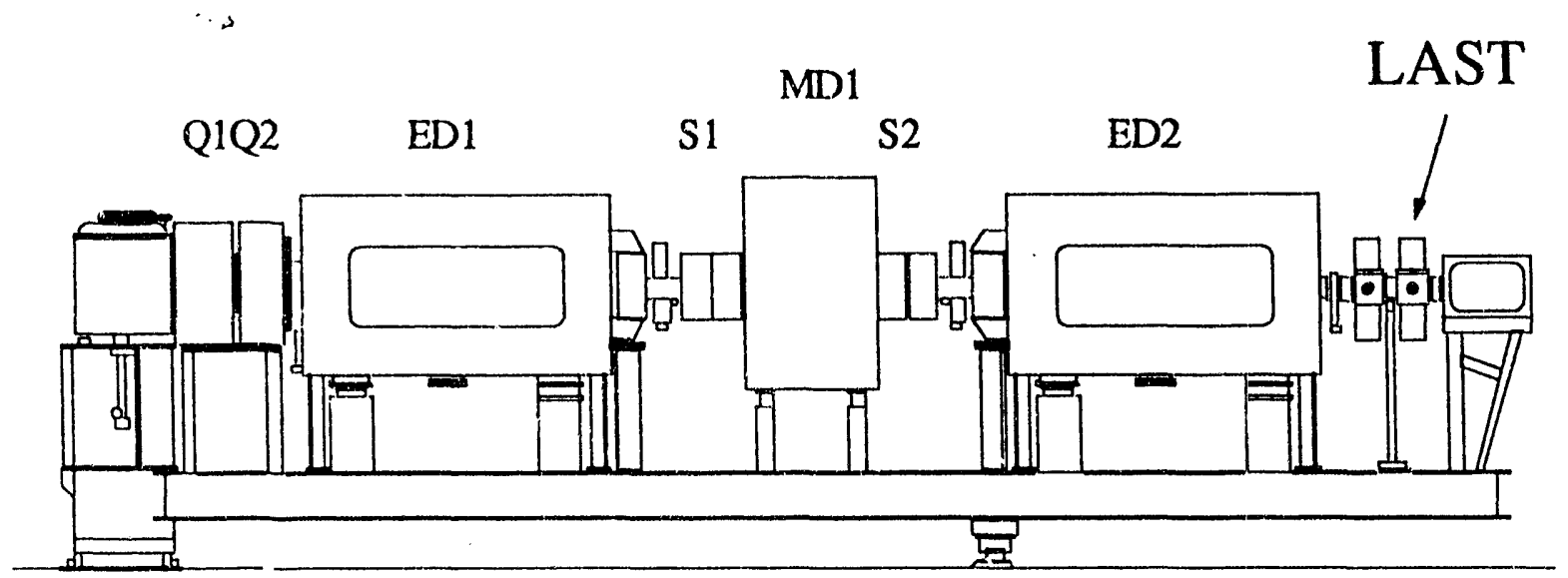

Figure 4. The LAST Detector Mounted at the Focal Plane of the FMA.

We are currently trying to improve the light output by : (1) testing new scintillator materials, (2) testing new foil mounting techniques, and (3) testing the effect of reflectant applied to the interior of the chamber. The durability of the foils which is already very good can be improved by irradiating the solution before the foils are poured. This produces cross-polymerization in the plastic and causes the foils to retain the active scintillator material longer.

In the next weeks the LAST chambers will be taken to Argonne National Laboratory for the first in-beam testing of the full scale chambers. If modification are required these may be done at Argonne or at the University of Florida. A full set of engineering drawings are reproduced on the next few pages for anyone interested in the detailed design. 


\section{0 - A New 16-Segment Neutron Detector}

A.V. Ramayya, E.F. Zganjar and R.B. Piercey

A new 16-segment neutron-multiplicity detector has been designed for use at the Argonne Fragment Mass Analyzer (FMA). The design is based on studies and tests done over the last ten years in collaboration with Köln, Vanderbilt, LSU. The new design, in addition to having more sectors than any previous design, is based on independent elements. This should allow a more flexible detector than was achieved in earlier designs.

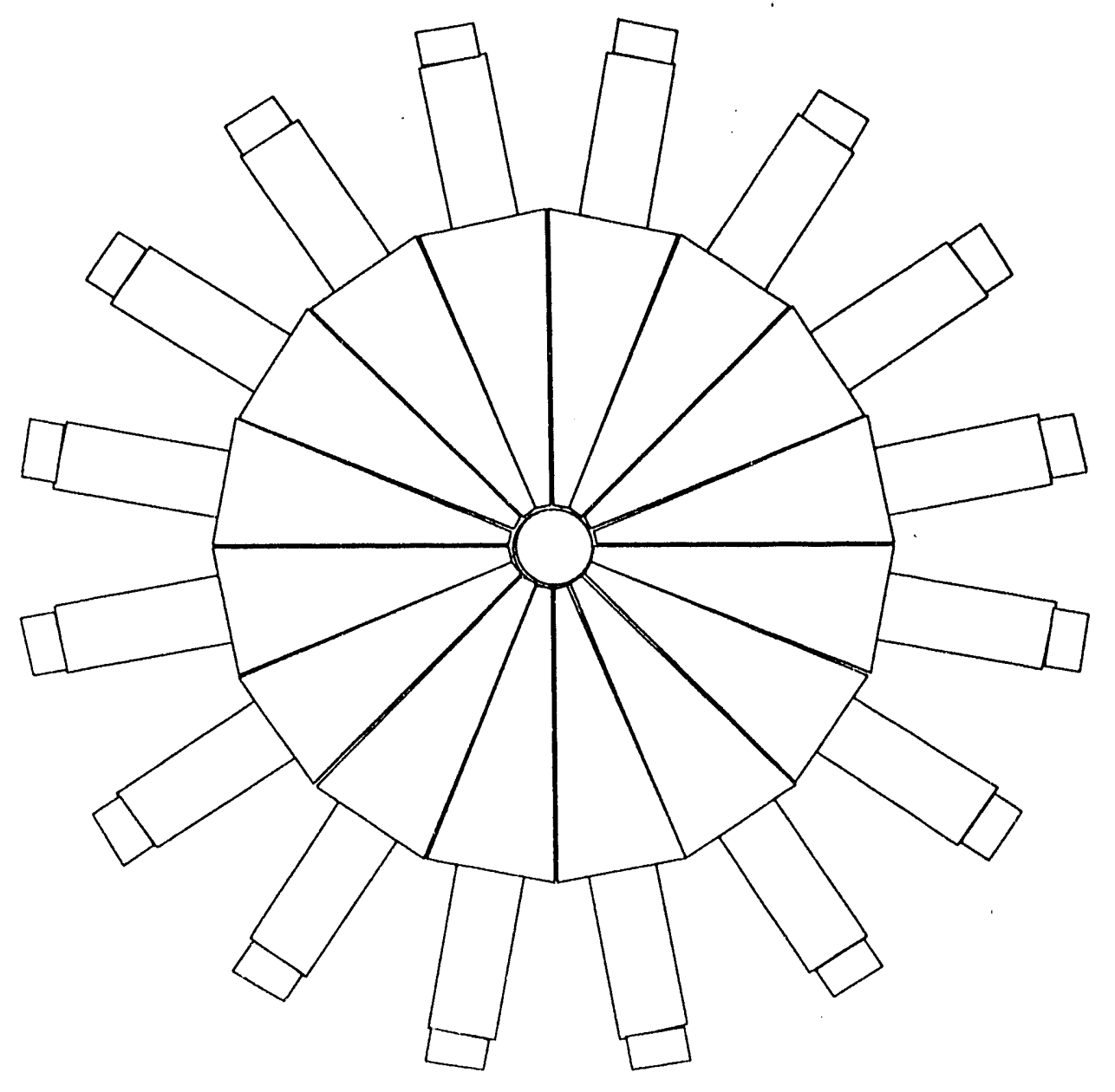

Figure 1. The New 16-Segment Neutron multiplicity detector. 
A diagram of the new detector is shown in figure 1. Each of the segments is filled with liquid scintillator and is view with a photomultiplier. The signals from the photomultipliers is processed to differentiate between neutron pulses and gamma-ray pulses. The neutron-gamma discrimination is accomplished by integrating the charge in the PMT output over short and long timescales. The technique and a schematic of the electronics is illustrated in figure 2. By fanning out each signal and delaying one with

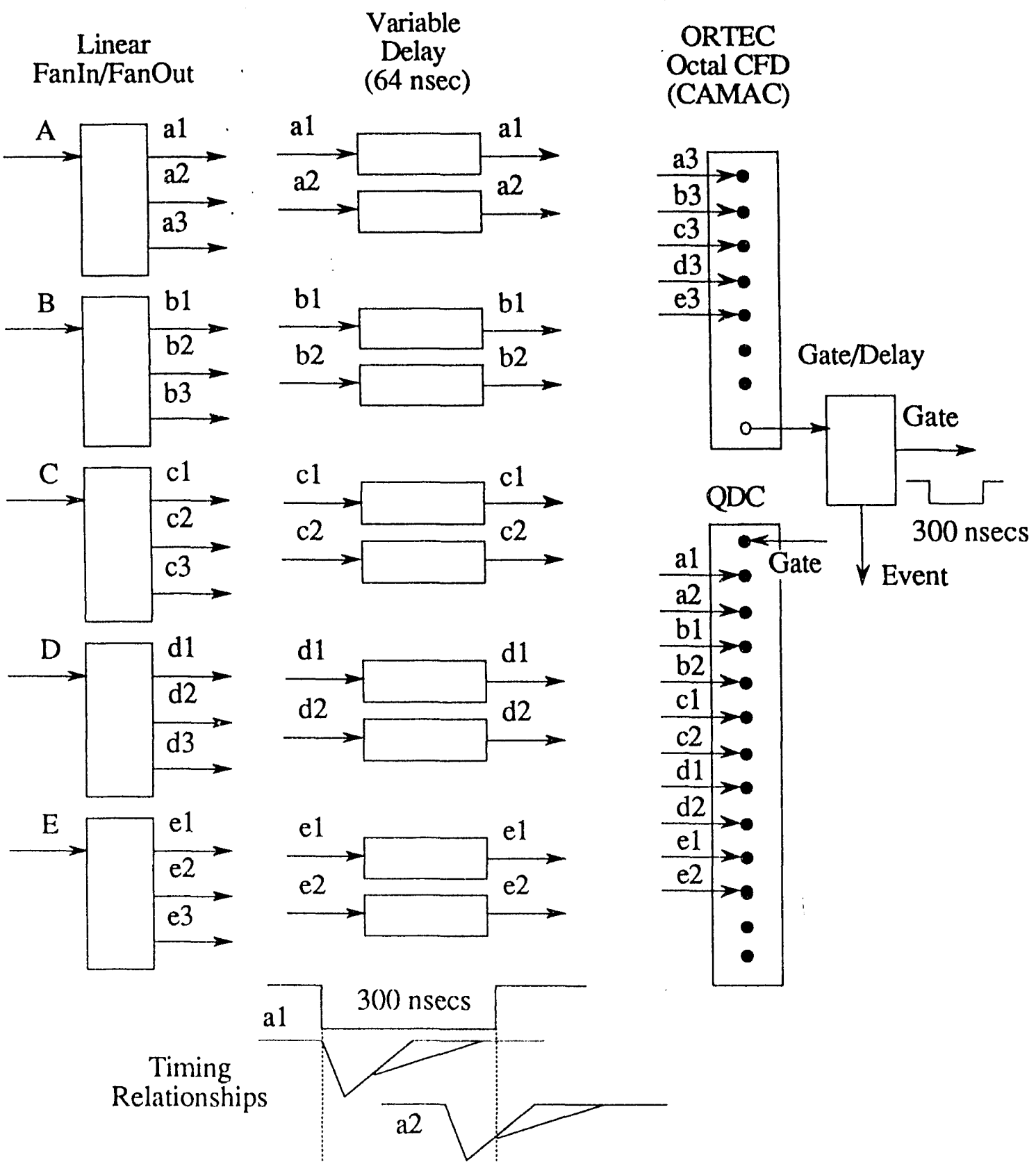

Figure 2 the Neutron-Gamma Discrimination Electronics 
respect to the other, only one wide gate is required to gate both the long integration and the short integration.

A test segment has been constructed and the preliminary tests indicate excellent neutron-gamma discrimination. The separation between neutrons and gamma-rays that can be accomplished is illustrated in figure 3 .

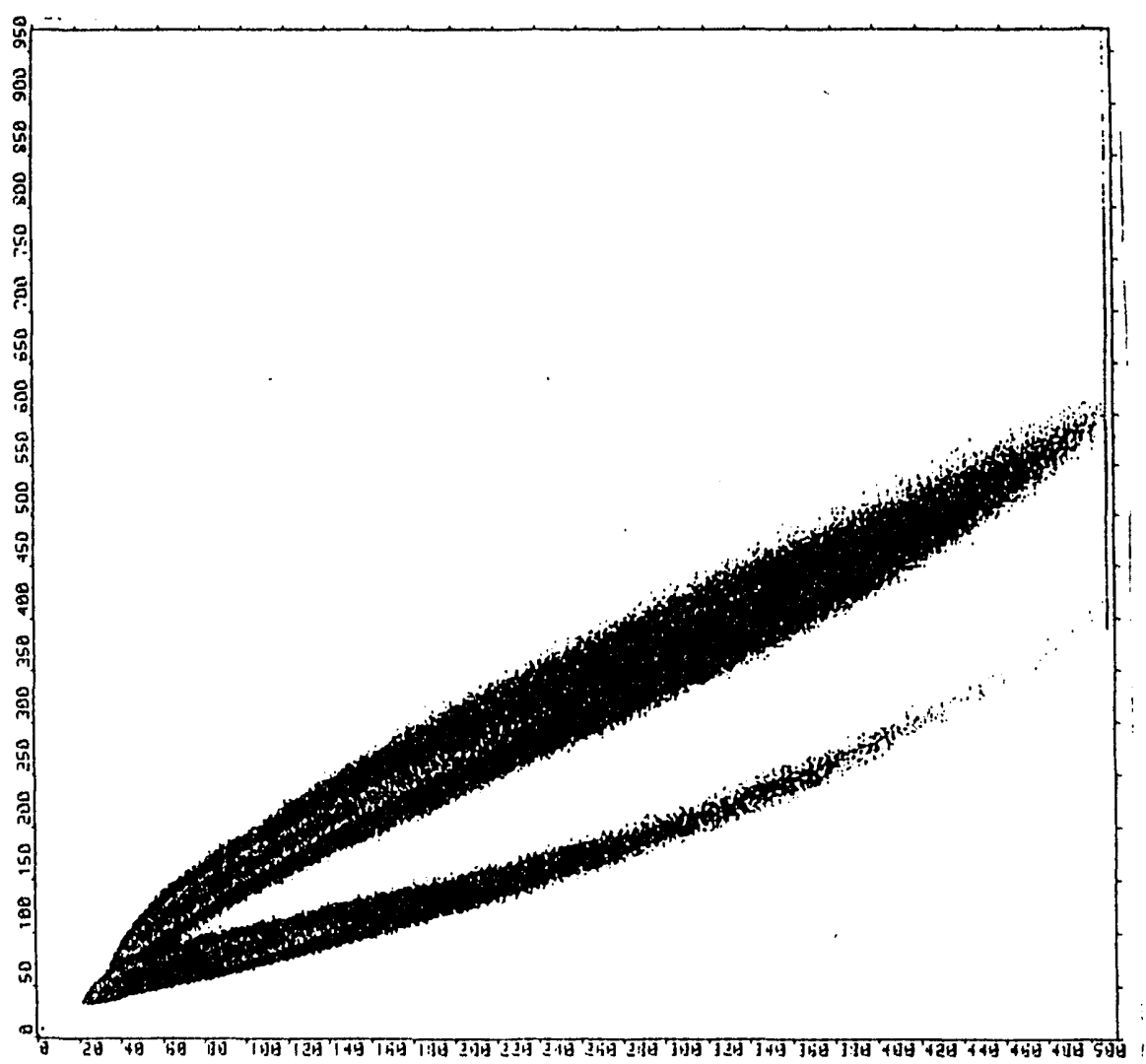

Figure 3 Prompt Charge versus Total charge for Gammas and Neutrons

The independent-segment design will allow enough flexibility to configure the neutron detector array with most other detector-target geometries. The 16-segment version will also provide a much improved multiplicity resolution as compared to the original 5segment PANDA design. 


\section{0 - High spin states in ${ }^{72} \mathrm{Ge}$}

A.V. Ramayya, J.H. Hamilton, L. Chaturvedi,J. Kormicki, R.B. Piercey

In April, 1989 we performed an experiment at ORNL to continue our studies the high spin states in ${ }^{72} \mathrm{Se}$. The data analysis is currently in progress. 


\section{SECTION-II PUBLICATIONS AND REPORTS}

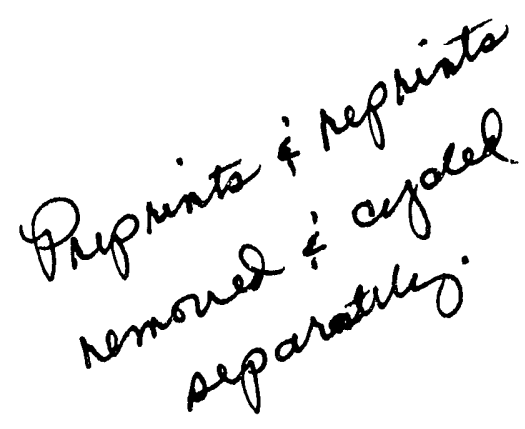




\section{SECTION-III INVITED TALKS, CONFERENCE PRESENTATIONS AND OTHER TRAVEL}
(a) - Argonne Fragment Mass Analyzer Workshop, Argonne Illinois
(b) - Argonne Pre-town meeting, Argonne Illinois
(c) - Invited colloquium at Mississippi State University, Starkville, Mississippi
(d) - Invited colloquium at Marshall Space flight Center, Huntsville, Alabama
(e) - 1988 IEEE Nuclear Science Symposium, Orlando, Florida
(f) - 1988 Meeting of the American Astronomical Society, Boston Mass 


\section{SECTION-III INVITED TALKS, CONFERENCE PRESENTATIONS AND OTHER TRAVEL}
(a) - Argonne Fragment Mass Analyzer Workshop, Argonne Illinois
(b) - Argonne Pre-town meeting, Argonne Illinois
(c) - Invited colloquium at Mississippi State University, Starkville, Mississippi
(d) - Invited colloquium at Marshall Space flight Center, Huntsville, Alabama
(e) - 1988 IEEE Nuclear Science Symposium, Orlando, Florida
(f) - 1988 Meeting of the American Astronomical Society, Boston Mass 


\section{SECTION-IV PERSONNEL}

Rodney B. Piercey, Associate Research Scientist

Zorn Milosivich, UF Graduate student

Tomas Dybler, Exchange Student, Royal Acamdemy of Sweden

Peter Agnvall, Exchange Student, Royal Acamdemy of Sweden

We would also like to mention the many man hours of technical support supplied by the Space Astronomy Lab at no cost. 


\section{Appendix-A Student Report, Tomas Dybler}

\section{Two \& Three-Dimensional Plotting Of Gamma-ray Data.}

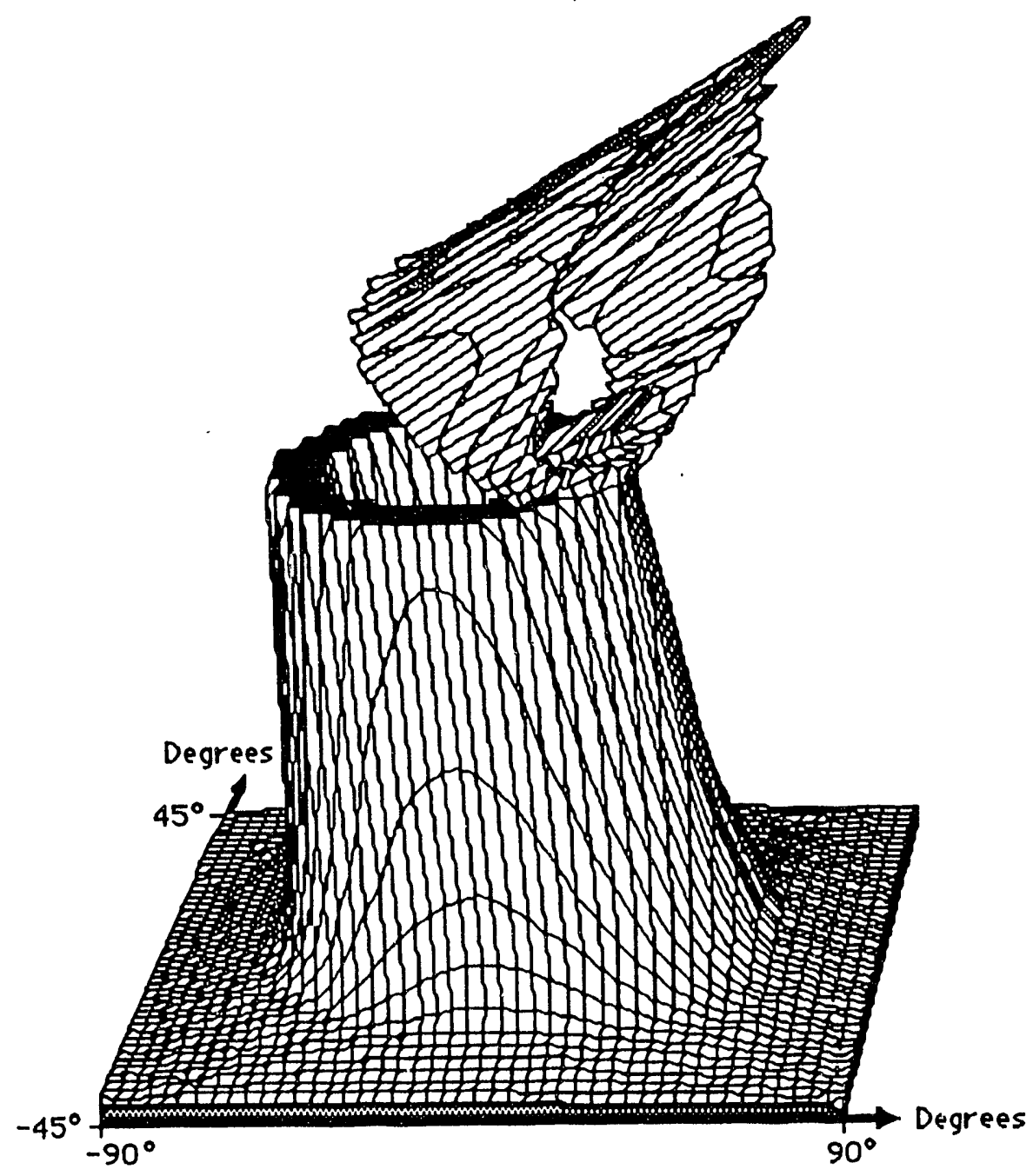

\begin{tabular}{|lll|}
\hline Name & $:$ & Tomas Dybler. \\
Date & $:$ & Summer 1988. \\
Advisor & $:$ & Dr. R.B. Piercey. \\
Location & $:$ & $\begin{array}{l}\text { Space Astronomy Laboratory, } \\
\text { University Of Florida, Gainesville. }\end{array}$ \\
\hline
\end{tabular}




\section{Table of Content}

Introduction.

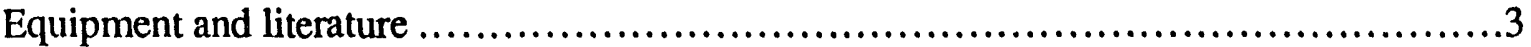

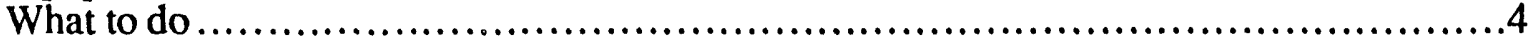

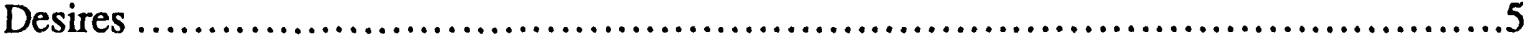

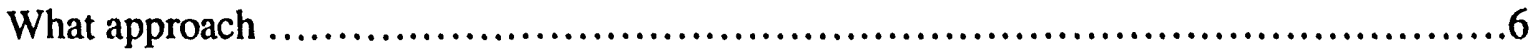

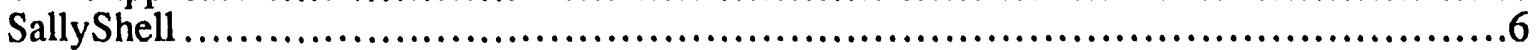

Beginning.....................................................................6

Debugging .........................................................................6

Difficulties ..................................................................6

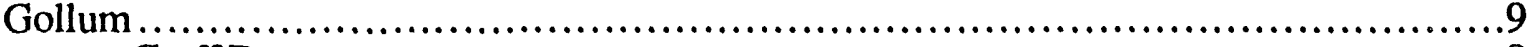

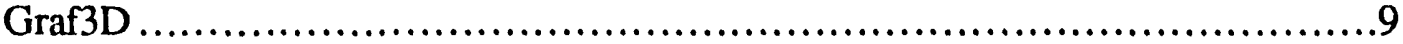

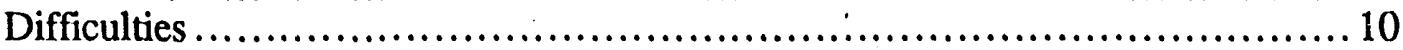

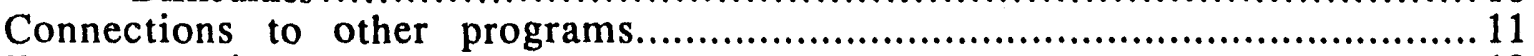

Future extensions .................................................................... 12

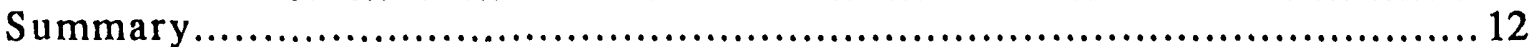

\section{Appendix A: (Flowcharts)}

The structure of the flowcharts........................................................ 13

TopLevel............................................................................... 14

MainEventLoop ................................................................. 15

DealWithMouseDowns.......................................................... 16

DoMenuCommand..................................................... 17

HandleFileMenu ................................................. 18

HandlePlotMenu .................................................. 19

HandleWindowsMenu ........................................ 20

HandleThreeDMenu............................................. 21

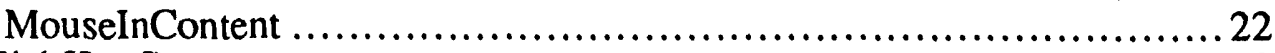

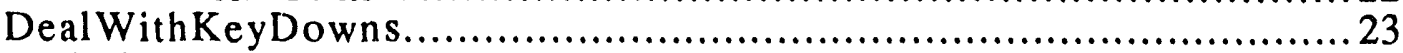

DealWithActivates .......................................................... 23

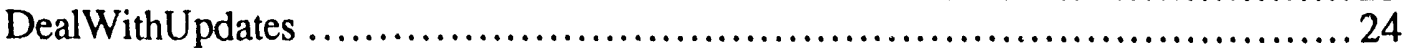

Appendix B: (Photographs)

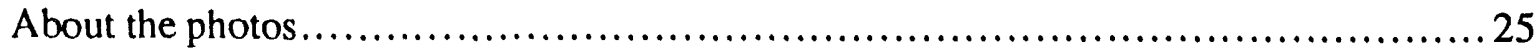

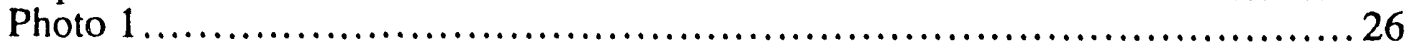

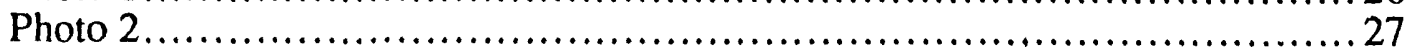

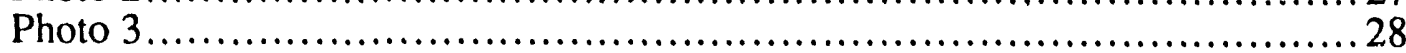

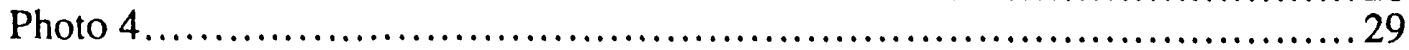

About the Outside : The

picture is a 3-D perspective plot of a pseudo image of a small sector of

the field of view of an imaging gamma-ray detector. The PlotMin $=14$

counts, PlotMax $=16876$ counts with a $43^{\circ}$ angle between the gamma-

ray source and the detector axis. An artistic enhancement has been used

to cut and tilt the data at 8431 counts . 


\section{Introduction}

This report is going to try it's best to describe and explain a Master thesis work done by me, Tomas Dybler, during the summer of 1988.

I am studying computer science and engineering at the Royal Institute of Technology in Stockholm, Sweden. My education there will lead to a Master of Science degree.

When it was time for doing the Master thesis work I contacted professor Rune Lindgren at the department of engineering sciences (aerospacebuilding) in Gainesville, Florida. Professor Lindgren has helped other students from Sweden to come to the University of Florida for their Master thesis work. It turned out that he had the perfect task for me :

Two-D and three-D color plotting of gamma-ray data on a Macintosh II - computer. The task was perfect because it combined my interest in graphic display techniques with my interest in human-computer interaction. This task was suggested by Dr. R.B. Piercey at Space Astronomy Laboratory of the University of Florida.

Before the travel to Gainesville I tried to find out what was previously done in the subject and what different problem solving techniques there was that could be used.

There are some people I would like to thank:

- My girlfriend Ann-Marie Brorsson for taking care of me.

- Professor Rune Lindgren for helping me to come here.

- Dr. R.B. Piercey for always having an idea.

- Jack McKisson for 'wild' discussions and handy tips.

- Drew Weisenberger for providing me with gamma-ray data.

- Dr. Penny Haskins for helping me find a workspace.

\section{Equipment and literature}

\section{The literature :}

Computer graphics \& MacIntosh specifics :

- 'Computer Graphics' by Donald Hearn and M. Pauline Baker published by Prentice-Hall. This is a good book covering general aspects of computer graphics.

- 'Computer Graphics - A programming approach' by Steven Harrington published by McGraw-Hill. This book concentrates on different graphic algorithms.

- 'A Characterization of Ten Hidden-Surface Algorithms' by Sutherland, Sproull and . Schumaker. This is an article published in Computing Surveys, Vol 6, No. 1, March 1974.

- 'Object oriented programming for the MacIntosh' by Kurt J. Schmucker published by Hayden 1986. This book covers different object-oriented languages, specially object-Pascal and MacApp. It also contains some information specific for the MacIntosh, such as the MacIntosh Standard Interface guidelines.

- 'Pascal Procedures - Understanding Graf3D' by Scott Berfield.This is an article published in MacTutor, March 1987. The article describes how to use and understano the Graf3D- package available for MacIntosh.

- MPW Pascal Reference Manual Version 2.0.1 


\section{The hardware equipment :}

A Macintosh II with :

- color screen

- 2 MegaByte memory capacity

- 80 MegaByte harddisk

- ImageWriter printer (Black \& White)

- LSi terminal connected to the modem-port on the Mac II

\section{The software equipment :}

Software available for the Mac II, including :

- MPW (Macintosh Programmer's Workshop)

MPW is an environment specially suited for Macintosh programmers. There is a 'operating system' and a large 'toolbox' that have routines that can be called from a program. In the 'toolbox' there is especially two packages to mention :

- QuickDraw (supports screen-drawing)

- Graf3D (supports 3-D drawing using transformations to 2-D (screen) and then routines from Quickdraw)

- Excel - a spreadsheet program that can be used to create three-dimensional data from functions, $E g: z=f(x, y)=\ln (x+y)$

- MacDraft, MacDraw and Superpaint - programs that can be used to manipulate pictures created elsewhere.

- Image $.51 \mathrm{C}$ - a program that can be used for animation of picture-sequences.

- Microsoft Word - the program with which this report was written.

\section{Other equipment :}

Camera-equipment (Used when taking pictures of the screen) :

- Pentax A-3 camera

- Star-D Tripod

- 100 ASA Kodak-film

\section{What to do}

What they needed at SAL (Space Astronomy Laboratory) was a way of viewing and manipulating their gamma-ray image data. The gamma-ray data is in the form of a matrix. For each data-set there is a row-length and a column-length, the size of the matrix is : column-length $x$ row-length". The column is called X-channel and the row is called the Y-channel. The value $[\mathrm{v}=$ Matrix ( X_Channel, Y_Channel $)]$ is a whole number and the unit of the number is 'counts'.

There are several possible ways of viewing the matrix :

1) Take one row in the matrix and plot it as a histogram $[z=f(y)]$. 2-D data mapped to 2-D screen-representation.

2) Take the whole matrix and plot it as a density plot $[z=f(x, y)]$. 3-D data mapped to 2-D screen-representation.

3) Take the whole matrix and plot it as a perspective plot $[z=f(x, y)]$. 3-D data mapped to 3-D screen-representation. 
A program, Kmax, already existed that could view the data in the same way as described in 1) above - that is - as a histogram. But Kmax could do a lot more then viewing the data, for instance :

- communicate with the hardware through a special suited s-quence language.

- gather new data from the gamma-ray detector direct from the program.

- compute new histogram from several already existing histograms.

- compressing existing histogram

The two first mentioned things goes under the label data-acquisition.

Data-acquisition and the histogram-viewing was already done so what was left? - density and perspective viewing of the data!

\section{Desires}

Plotting-capabilities: We want to be able to

- view the data by drawing a density plot (and perspective if there is enough time).

- choose an area in the density plot and then expand that area (like a looking glass).

- choose a range of values that we want to look on.

- view all data available by choosing a 'show all' - command.

- set axes and label the data.

I0-capabilities: We want to be able to

- read in text-files containing the data.

- read in a file-structure special for density plots.

- save the data-structure for density plots.

Interface-capabilities : We want to be able to

- give the user as much freedom as possible by giving him/her the largest possible set * of commands to choose from at any given time.

- scroll around in the density plot.

- drag windows, resize windows, zoom in/out windows ...

- arrange windows - tile them and stack them.

- manipulate things directly with the mouse.

In short : we want to, as much as possible, follow the MacIntosh Standard Interface guidelines (See Kurt Schmuker)

In general :

My program should look and behave the same way Kmax does. A user familiar with Kmax should not feel lost in my program. 


\section{What Approach}

I studied Kmax and discussed different approaches with Dr. Piercey for how to start. We decided to start with a shell application that was already being used at SAL. The shell allowed us to concentrate on the plotting algorithims and not have to re-invent the wheel.

\section{The Shell}

\section{Beginning :}

In the beginning I concentrated on taking out things that would not be needed in my program in the future. I tried to keep the program error-free and working so that I wouldn't have something erroneous to begin with when starting to add new things. This strategy also gave me a chance to become familiar with the shell code that had already been written.

When I had stripped everything I possibly could, I started to rebuild the program by adding piece by piece, trying to make one piece error-free before adding the next.

\section{Debugging :}

One very helpful assistant during the work was the unreliable but sometimes working old termina! (LSi) that was connected to the Mac II's mouem port. The modem port is opened in the beginning of the program and it is simple to write things to that port for display on the terminal. It turned out to be very handy to write out certain data and procedure begins/ends because that made ir easy to follow the program execution. If the Macintosh would break down for some unknown reason, you could see exactly where by locking ori the LSi-terminal. This arrangement saved a lot of blood and tears and can be warmly recommended.

\section{Difficulties :}

At different stages during the develcpment of this program we encountered different difficulties. I would like to take a closer look on three of them here :

- Memory limitations and constraints

- Fast plotting

- Human-Computer interface 


\section{Memory limitations and constraints}

How could we handle large data-sets ?. MacIntosh recommend that no structured type is larger then $32 \mathrm{KBytes}$. The most obvious record for this kind of data would be :

Type MatrixType $=$ Array [1..NrOfRows , 1..NrOfColumns] Of LongInt;

A variable of this type ( aMatrix : MatrixType; ) would access a matrix-element on row ' $a$ ' and column ' $b$ ' in the following way : value $:=$ aMatrix[a,b].

If we want to keep this structure within the size of $32 \mathrm{~K}$ - how large could the matrix be ? Answer : $90 \times 90 \quad$ ( SQRT(32768/4) , 4 = bytes per LongInt)

How large is the gamma-ray data-matrix?

Answer : $360 \times 180 \quad(253,125$ KBytes $)$.

What we need is a new data-structure !. This is the chosen structure :

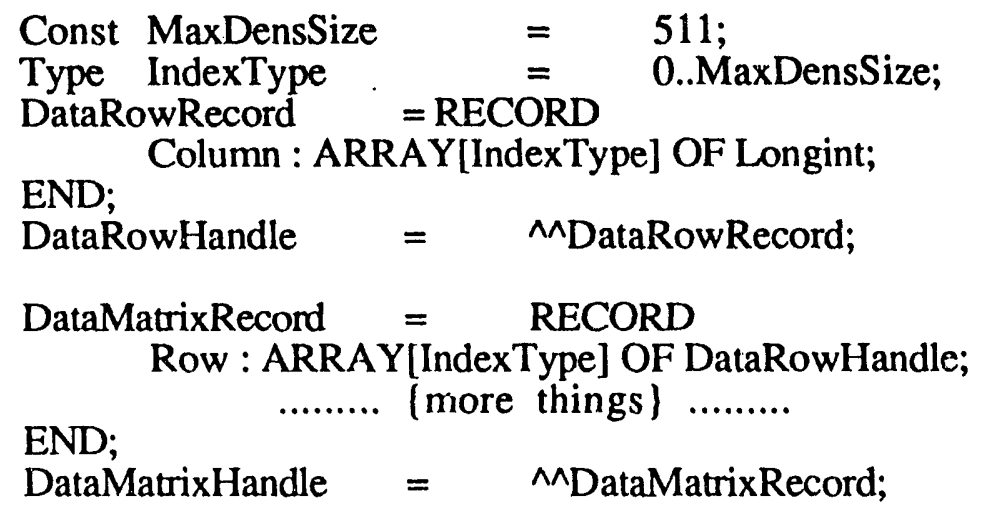

This is a picture of the data-structure :

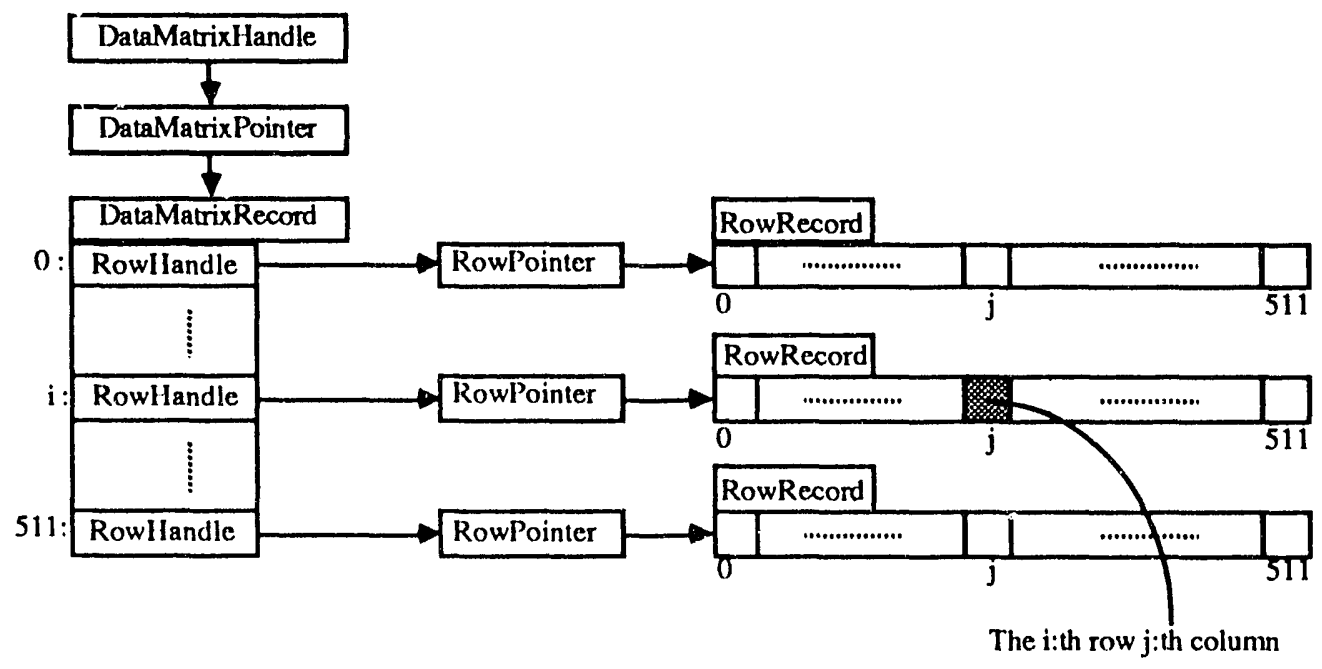

In fact this structure can handle a matrix $8192 \times 8192$ in size $(268,435$ MBytes) without any structured type being larger then $32 \mathrm{~K}$. But we only have a 2 MByte memory. 
Limited memory-space also have to be considered when reading in large data-files. There are two different filetypes that the program can read in : the Dens filetype and the Text filetype.

The Dens filetype has a 512 Byte header followed by the actual data-set. The header contains essential information specific to the data-set, such as 'DataXLength' and 'DataYLength' (The size of the Matrix that the data-set makes up) and several other things. Just by reading in the header we could calculate how much memory we would need if reading in the rest of the file - if we don't have enough memory we alert the user and cancel the 'read-in'.

With the Text filetype on the other hand we do not have the 'information-header' what we have is two numbers in the beginning of the file telling us the matrix-size (DataX/YLength). This is enough for knowing the memory-space needed - if there is not enough memory-space available we alert and cancel the operation. (Works the same way as the Dens filetype).

The difficulty arise when having text-files larger then $32 \mathrm{~K}$. Why ? - well, since the file consists of text (not numbers) a conversion has to be done (The text ' 1 ' and ' 7 ' has to be interpreted as the number 17). We can not read in all text in a buffer in order to convert to numbers (Because that buffer would be larger then $32 \mathrm{~K}$ ). We solved this problem by taking pieces less than $32 \mathrm{k}$ from the file into a text-buffer and then convert from the text in that buffer to numbers in our data-structure (above). When we came close to the end of the text-buffer it had to be filled up with another piece of the text-file again. This solution does not limit the maximum size of a file.

\section{Fast plotting}

An example : we have a data-set of Xlength $=360$ and Ylength $=180$ (The gamma-ray data size). This is $360 \times 180=64800$ values. Each value owns a square on the screen (big or small) and that square has a color determined by the value. If we want to show all the data we would have 64800 very small (since they all have to be on the screen) squares. Now the time-consuming part is when you actually paint all these tiny squares. I have done three things to speed up the density-plotting :

1) I have been very careful when programming the part of the plotting-routine that has to be gone through 64800 times since a small change here can mean a lot of time won or lost.

2) I go through the data before the actual plotting and find out the color that is going to be the most used color in the plot. I start the plotting by painting the background with the found color, which is done by painting one rectangle.

During the plotting-routine I do not take any action at all when I find a value that would get the same color as the background-color.This saves me from painting at least $1 / 8$ of the 64800 small squares. In some cases, when you have a very dominant color, this could increase the speed maybe ten times. 3) When I go through the data I gather 'runs' of values represented by the same color and paint them only when finding a value which would be represented by another color. For instance : instead of painting 17 small squares in a row with the same color this approach would paint 1 rectangle (made up by the 17 squares in a row).

A fourth thing to do is to save the addresses to the routines used in the critical part and call the routines through these saved addresses directly, preventing the address-lookup that normally is done every time a routine is called. This would save 64799 addresslookups per routine and could give us double speed. 


\section{Human-Computer interface}

Programming on/with the MacIntosh is really very different from programming any other machine - that is, as I see it, due to two main reasons :

1) The Event-Queue concept : When a user does something (press a key, do a mouse- click) this forms an event and the event is placed in a queue, waiting to get processed. The program you write has to deal with (and sometimes process) these events. The programmer never know in which order his program is going to be executed because that is decided by the user of your program.

2) The Standard MacIntosh Interface guidelines. In short this means : every application should look the same to a user. These guidelines are supported by the environment you use when writing your program. You should disobey the guidelines only when it's highly motivated. There could be many things said about these guidelines but one thing is clear: The user benefits from having a consistency from program to program. (He/She doesn't have to 're-learn' all the time).

question :

We had to experiment with the looks of the user interface. We got to a main

How should the display of the data go together with the MacIntosh-windows?.

We didn't find any direct answer but we came up with three main approaches :

1) Give the data the power: Whenever the user wants to view a data-set the program resizes the window to fit the amount of data the user wanted to see.

2) Give the window the power: If the user resizes the window that would change the amount of data the user could see. (This is the Stand. Mac. Interface approach)

3) Split the power between the data and the window. When the user has decided what data-set he/she wants to see, resizing the window will never show less than the user- decided data-set, although resizing could show more in which case the additional data is shown under a gray coating.

We chosed approach nr. 3 after testing all three.

\section{Gollum}

At this stage I was heavily influenced by the trilogy 'Lord of the ring' by Tolkien so the program was renamed after my favorite character 'Gollum'. The name-change also came from the fact that I now felt that it was time to try and plot perspective (or 3-D) plots. SallyShell that handled density plots were extended to show also perspective (3-D) plots.

\section{Graf3D :}

Graf3D is a package that supports 3-D drawing using transformations to 2-D (screen) and then routines from Quickdraw. E.g there is a routine called LineTo3D $(x, y, z)$ , when calling that routine with a 3-D point $(x, y, z)$, it transforms the 3-D point to a 2-D point $(x 1, y 1)$ and uses the quickdraw routine $\operatorname{Line} \operatorname{To}(x 1, y 1)$ to draw the line on the screen. In this package there are routines for drawing, rotating, scaling, translation. There are also routines for setting up 'the camera', that is, how you want to view and what space you want to view (Different perspectives ...). The article 'Understanding Graf3D' (see 'Equipment and literature') was really helpful since this package is badly documented. 
for free

I wanted to use the Graf3D-package since that would give me many things nearly

(Different perspectives and rotation-capabilities).

\section{Difriculties :}

The difficulties involving perspective 3-D plotting turned out to be :

- using Graf3D.

- how to solve the hidden-line removal problem in the 3-D plotting.

\section{Using Graf3D}

Every time the plotting-routine in Gollum is called it has to calculate the 3-D space to map to the 2-D screen area. The 3-D space is a 'view-box', limited by what you see in the 2-D density plot (XMin->XMax, YMin->YMax, ZMin->ZMax). Since the user can rotate this 'view-box' arbitrarily you never know which exact position the 'view-box' will have on the screen - but it's possible to calculate a 2-D rectangle enclosing every possible screen-mapping of the 'view-box'. How is this possible ? - Well, you get a sphere when rotating a 'view-box' arbitrarily in three dimensions and a sphere always maps to a circle in two dimensions. A circle in two dimensions is always enclosed by a rectangle (in fact a square rectangle). Now we know the limits of the screen-mapping and we know the 3-D space we want to view (the view-box), that makes it possible to use the routines in Graf3D to set up the camera. The user decides what kind of perspective he wants, how to rotate the view-box and how the plot should be drawn.

\section{Hidden-Line removal}

Hidden-line removal means that lines hidden by surfaces should be invisible from the viewers point of view. If they are visible it gives you a feeling of transparency and in some cases the plot gets very hard to understand. I decided io give the user the option of how the plot should be drawn - with hidden-line removal or without.

There are several algorithms that deals with removing hidden-lines. One of these algorithms seemed very well suited to this problem : 'The Painter's Algorithm', it's called that because it works the same way as an artist creating an oil painting. The artist starts with the background and then paint layer by layer until the oilpainting is finished. The new paint covers the old. The same way I would start with painting the surfaces farthest away from the viewer (the background) and paint the surfaces closest to the viewer at the end (the foreground).

Normally to use this algorithm you have to sort the surfaces from the background to the foreground but in my case the surfaces were already sorted. Since the user can rotate the 'viewbox' I have to find out where the back and front are. This leads to four different cases :

The matrix (data-set) is viewed from above : 

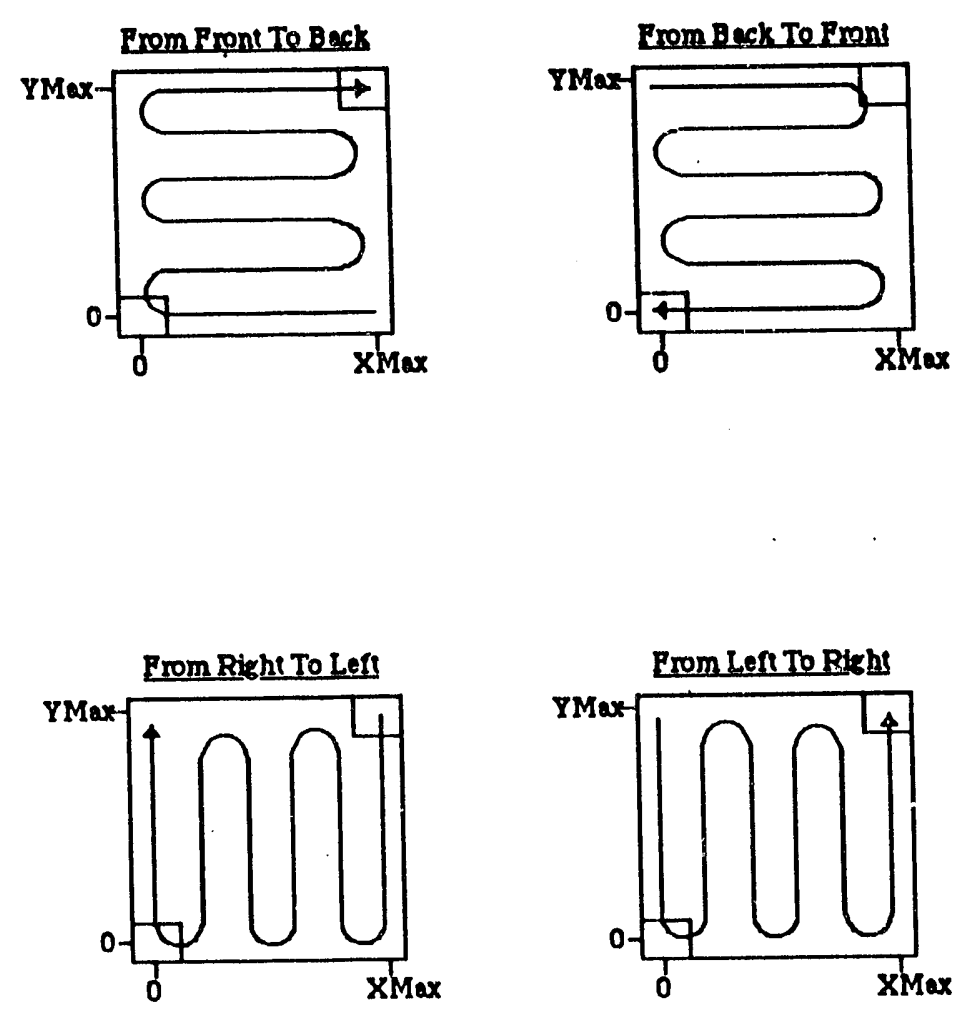

What I have done in my program is to split the drawing-routine into four cases (represented above). Depending on the y-rotation-angle I chose the corresponding case :

Y-rotation angle
$0^{\circ} \rightarrow 90^{\circ}$
$90^{\circ} \rightarrow 180^{\circ}$
$180^{\circ} \rightarrow 270^{\circ}$
$270^{\circ} \rightarrow 360^{\circ}$

\author{
Routine \\ From Back To Front \\ From Left To Right \\ From Front To Back \\ From Right To Left.
}

This solves the hidden-line removal problem.

\section{Connections to other programs}

Since the program can read in text-files we have opened bridges to many other programs, such as Excel, were it normally is possible to save data as text. You could enter a formula in Excel in order to create a data-set, save it as a text-file and then read it in to my program to be able to view the structure of the data-set.

Another way of interacting with other programs is by copying the plot you have in my program and then go into MacDraft (or MacDraw,MacPaint,SuperPaint....) and paste the plot.

Then you could rearrange the plot and finally let it go into a report (or into the bin).

There is another way of moving the plots : take a 'screen-picture' by doing 'cmd-shift-3' on the keyboard. Read this 'screen-picture' in to Superpaint (or other paint-program) and rearrange it as you want. (This is how the outside of this report was created). 
To take this interaction far you could even create a bunch of pictures and then have them animated. Create the pictures that together make up the sequence you want to see animated and then read them into the program 'Image 51C' in the order you want them 'played' - select movie-mode in one of the menus and you are there !!. (Could not include this in my report since it would demand a video-camera). 


\section{Future Extensions}

'A program is never finished' - I think that there is always something to change or add to a program. In this case there is a lot of things one could do to extend this program :

-Real-time rotating of the 3-D plot, maybe by having two scrollbars, one for the angle of rotation around the $\mathrm{x}$-axis and one for the $\mathrm{y}$-axis.

- Lightsource-shading of the surfaces in the 3-D plot. Surfaces hidden from the light-source are shaded darker than the surfaces hit directly by the light.

-The 3-D plot in color. It would be possible to color the 3-D plot according to the color-scale used in the density-plot by calculating intersections between the color-scale limits and the surfaces (E.g one surface would be painted in three colors if ranging through three colors in the color-scale).

-Quicker 3-D plotting : calculate the surfaces hidden from the viewer (Maybe this would turn out to be slower then the present technique used).

-Color-Quickdraw. Now only 8 colors are used in the density plot - Rebuild the program to use color-quickdraw and thereby get access to the whole colorspectrum. Let the user decide which colors to use in the color-scale. (Instead of eight different colors we could have eight different intensities of the same color, E.g from yellow to light-red).

-Animation. Why not ? - For instance (in 3-D plot) : set a start rotation point (xangle, $y$-angle) and a stop rotation point ( $\mathrm{x} 1$-angle, $\mathrm{y} 1$-angle) and let the program animate by rotating the plot from start to stop. To do this we need more power (and memory ?) because the next plot in the animation-sequence has to be calculated very quickly otherwise it would be no animation. The animation would give the user a very good feeling of how the data-set really looks like. (The animation idea came from using the animation-capabilitiy in the 'Image $51 \mathrm{C}^{\prime}$-program, with pictures of the plot taken in different angles)

-Talk. Use talk as an extended feedback to the user. E.g 'Welcome to Gollum territory'. The talk-capability had already been incorporated into the shell.

-Compute. The Shell already had a command called 'compute' where you can combine data-sets or calculate something and then store it into a new data-set. E.g you can add two curves (data - sets) together to create a new one. The Shell routines that do this could be modified to fit Gollum.

-Calibrate. Lets the user calibrate the data in Shell - could be incorporated in Gollum.

\section{Summary}

The work I have done at SAL has been very interesting and educational. It's interesting to work with something that nobody or very few have ever done before. Specially when we considered the different approaches to the human-machine interface, it felt like 'frontier-work'. It's also stimulating to try and see the extensions that could be done to my program. Since Dr. Piercey has invited me to continue my work at SAL I might be able to do some of these extensions.

Taking the photos of the screen was also a 'small adventure' - we didn't know at all how the outcome would be ! (see Appendix B).

Dr Piercey says that programming has two main aspects : first it's a creative and iterative process of designing the human-machine interface and second it's a engineering process to build up the structure of the program. I agree - and just that combination of art and engineering makes it very stimulating to work with the MacIntosh. 


\section{Appendix A : Flowcharts}

\section{The structure of the flowcharts}

This is what different things in the flow-charts mean :

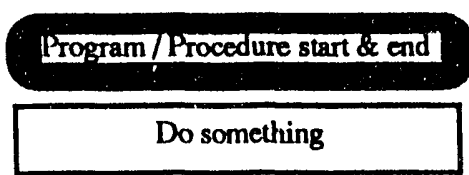

Procedure that is explained further

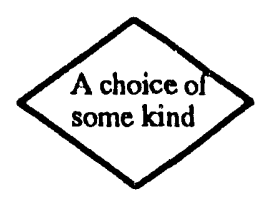

Control of flow

This is the structure of the flow-charts :

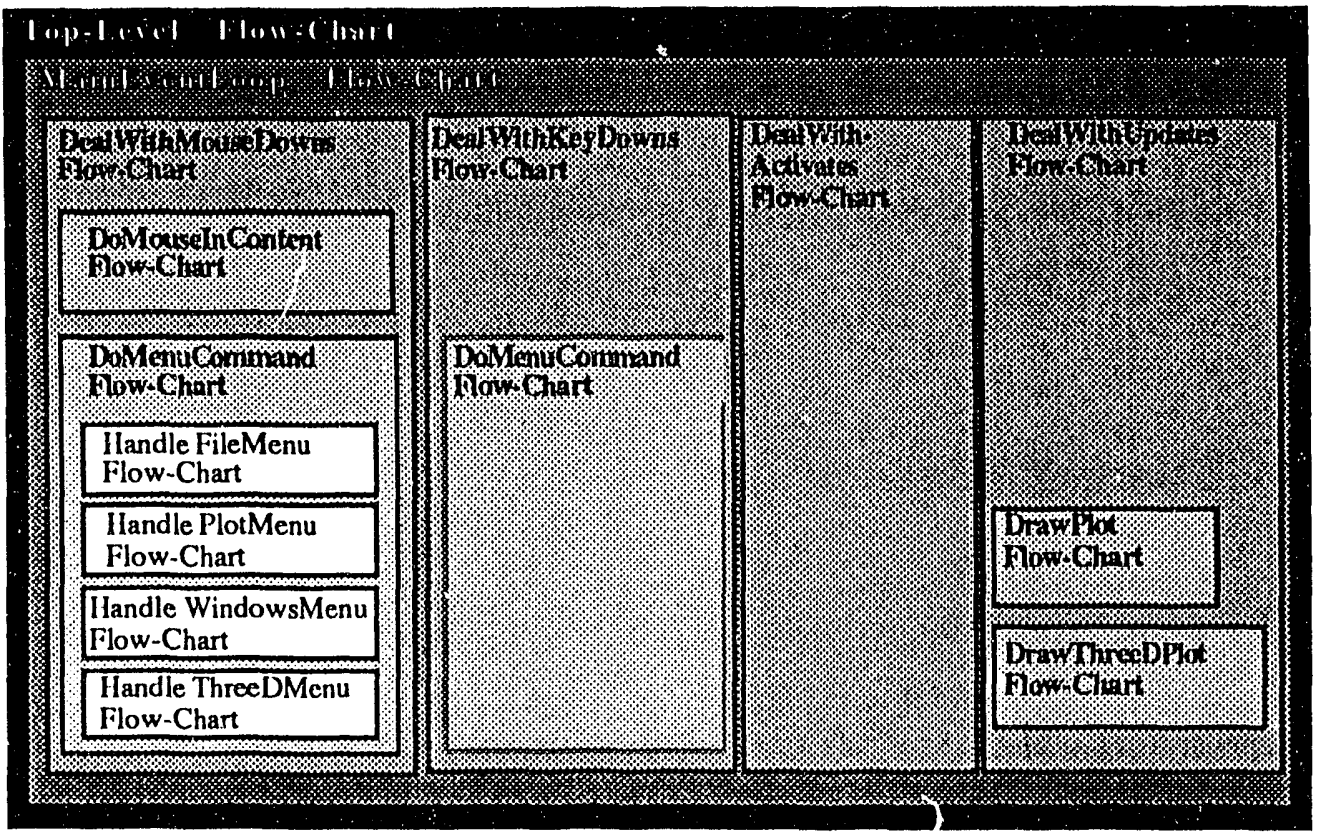




\section{TopLevel}

This is the outermost level of the program-flow

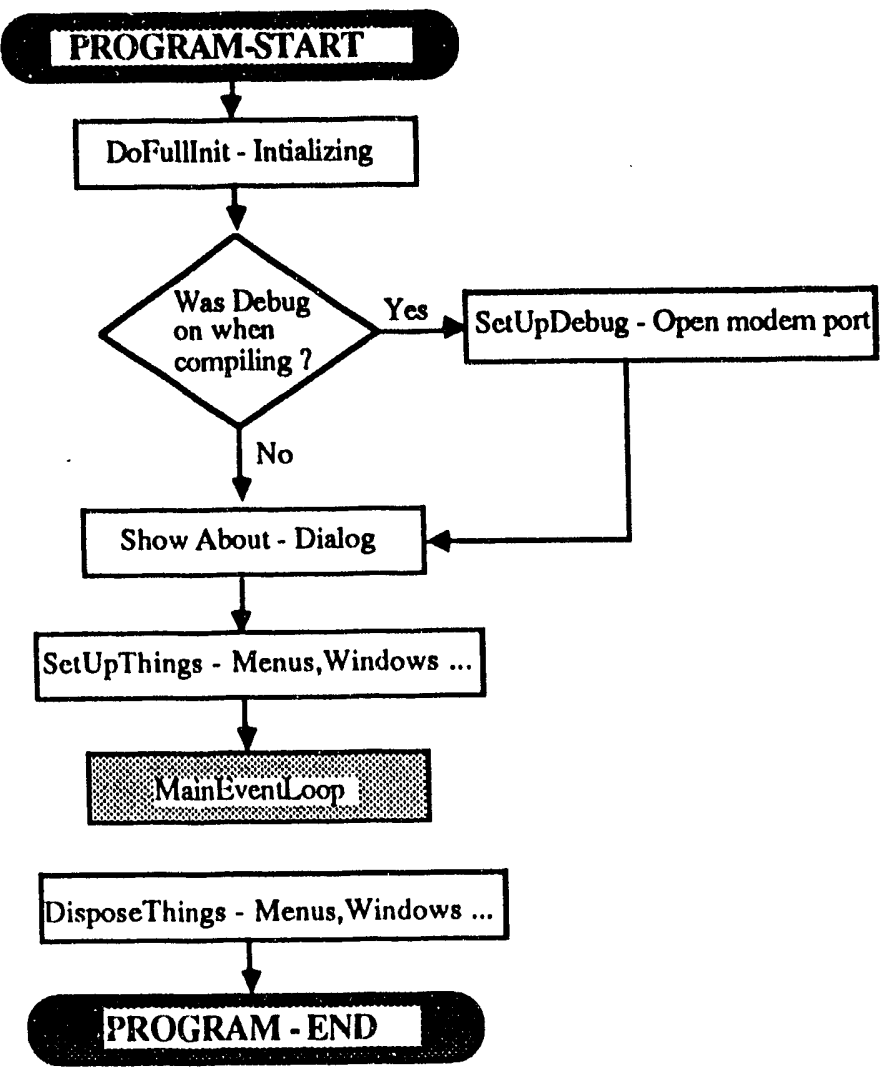




\section{MainEventLoop}

This is the MainEventLoop level of the program-flow

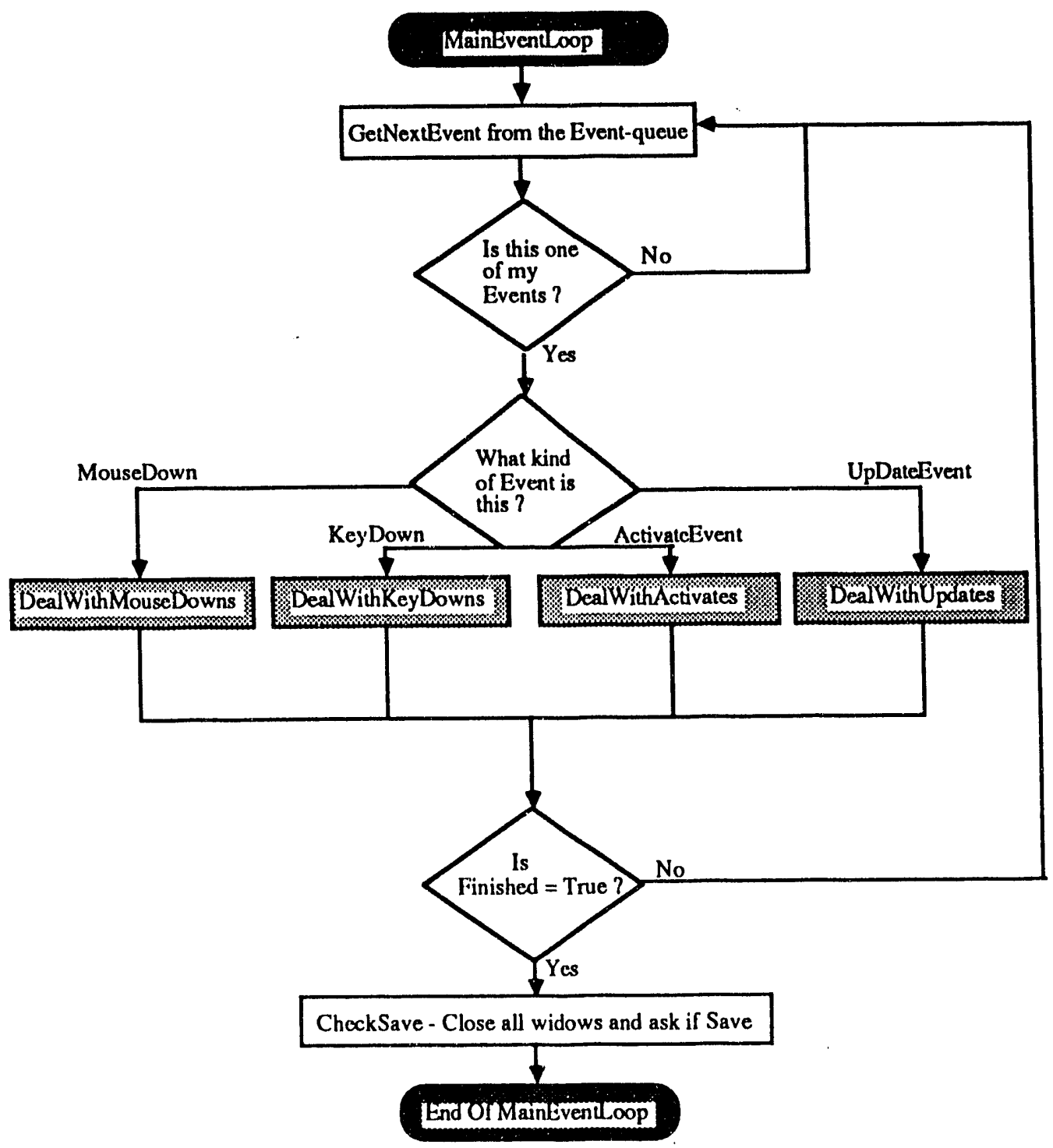




\section{DealWithMouseDowns}

This is the DealWithMouseDowns level of the program-flow

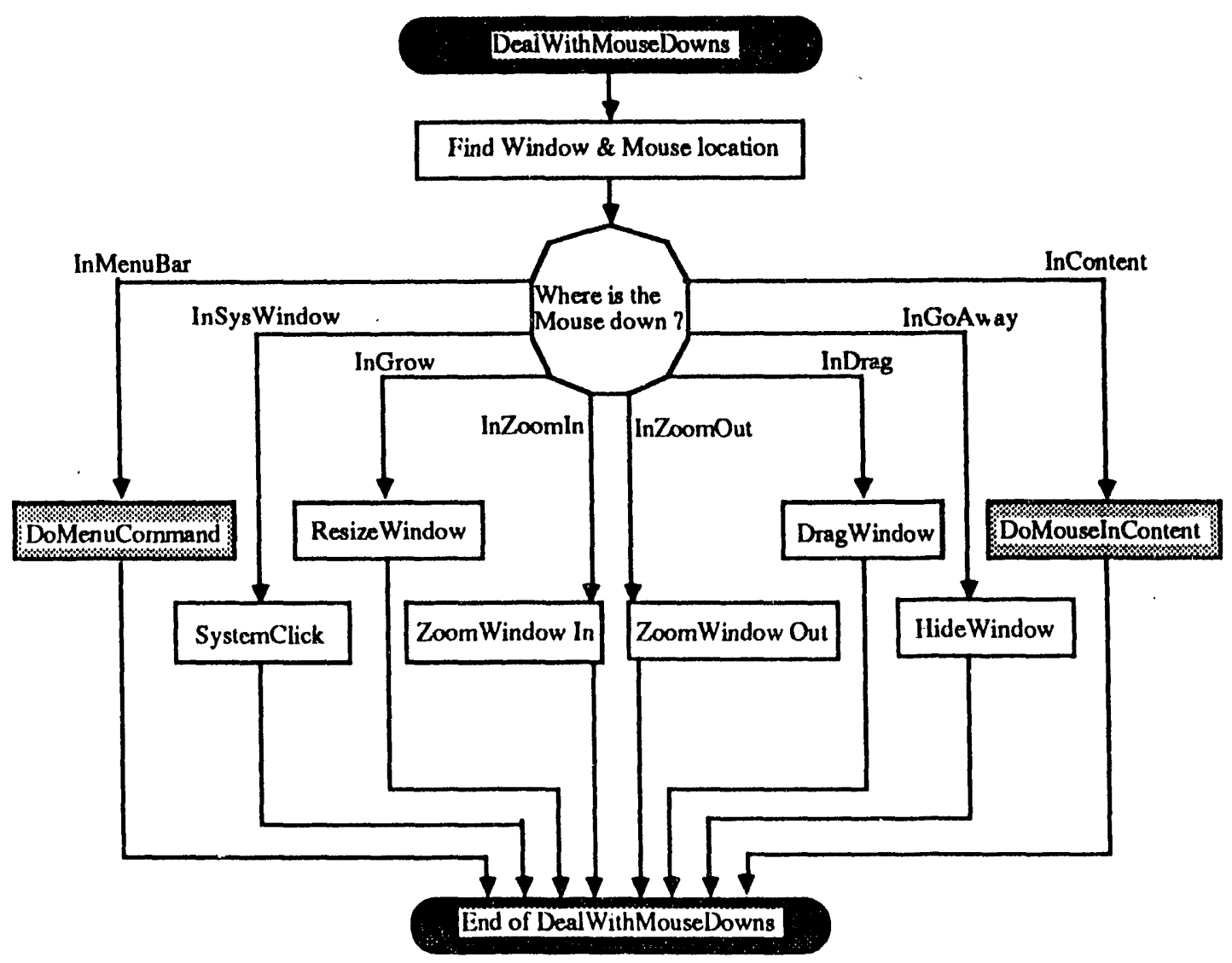




\section{DoMenuCommand}

This is the DoMenuCommand level of the program-nlow

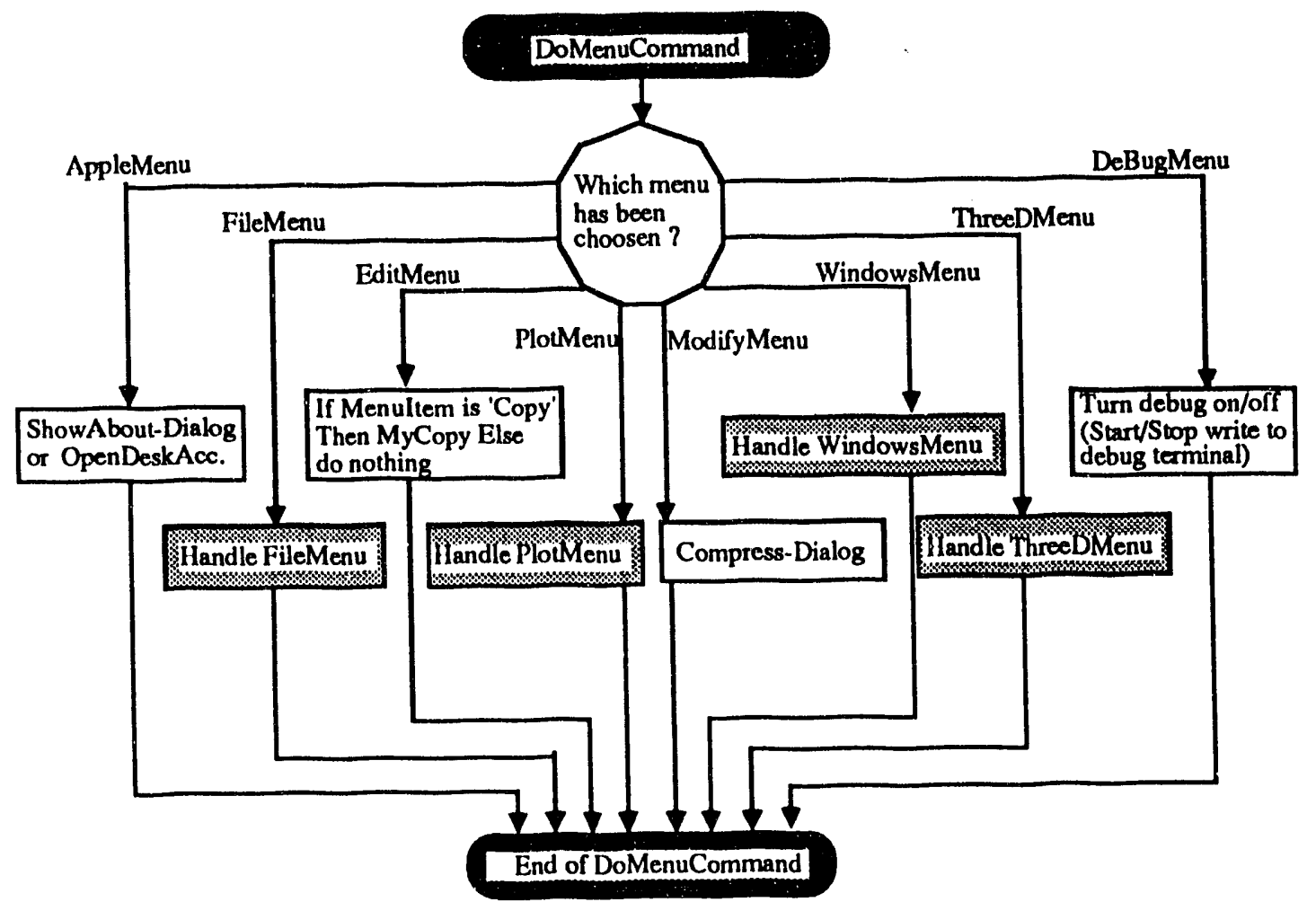




\section{HandleFileMenu}

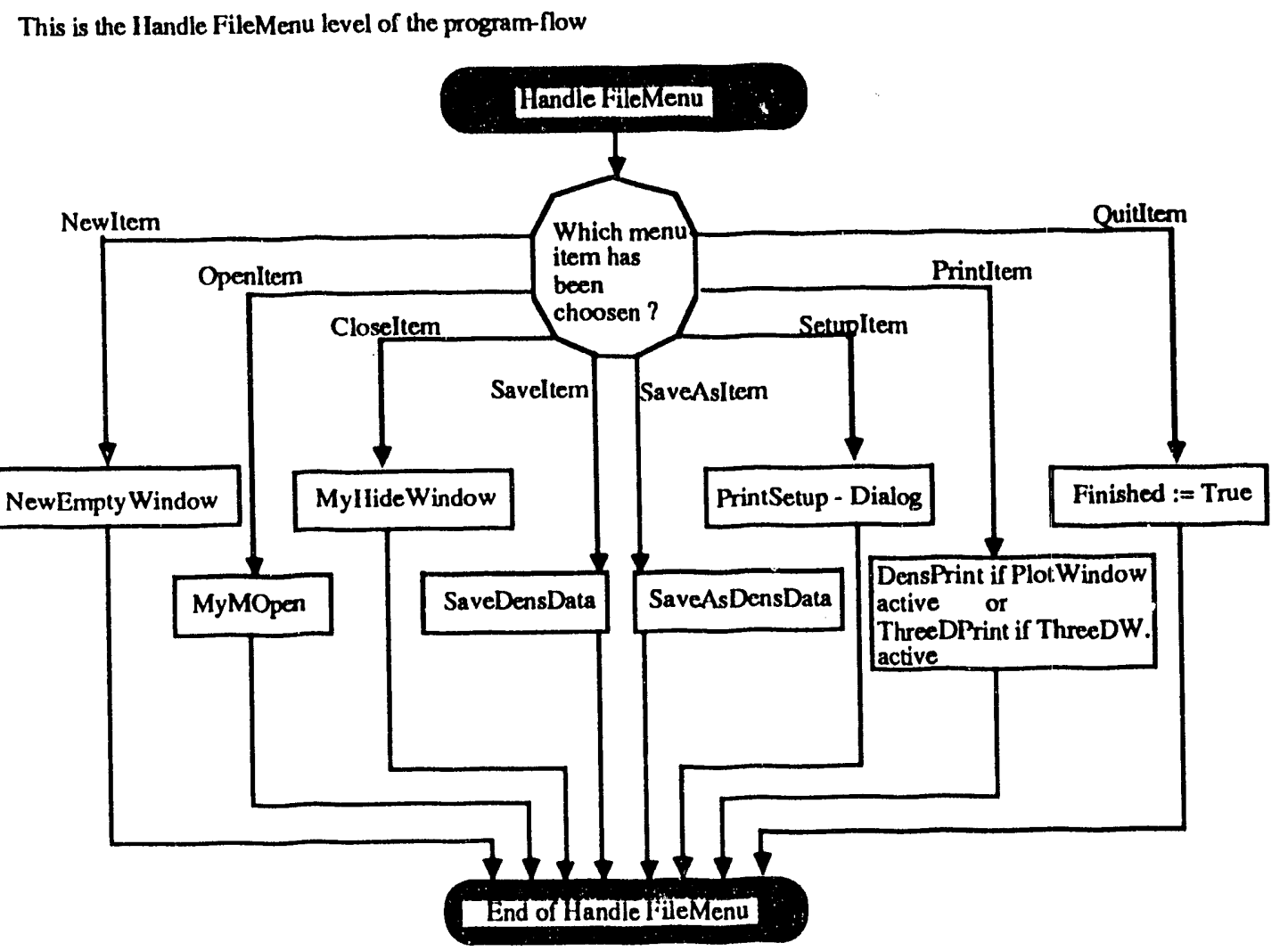




\section{HandlePlotMenu}

This is the llandle Plot:Menu level of the program-flow

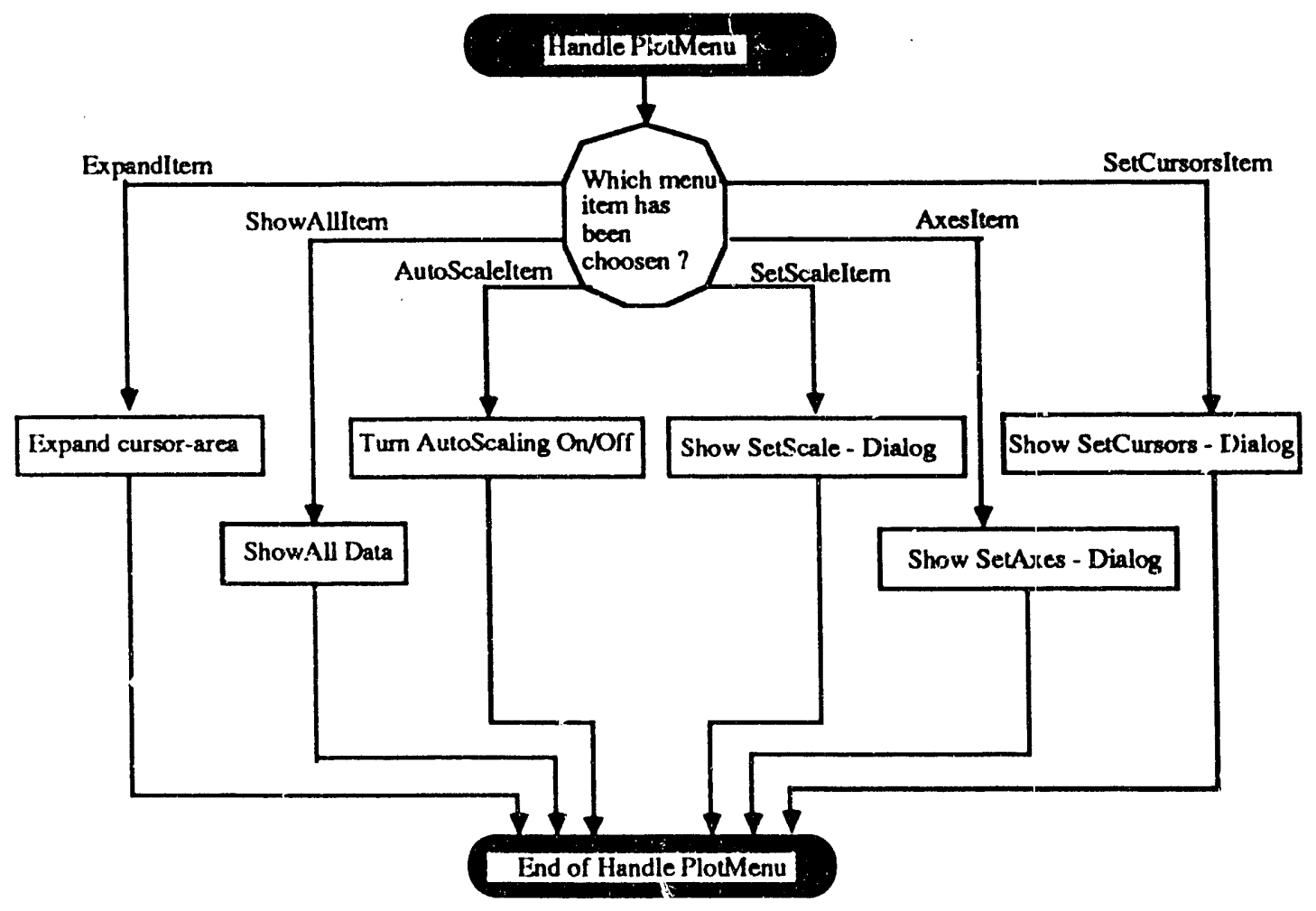




\section{HandleWindowsMenu}

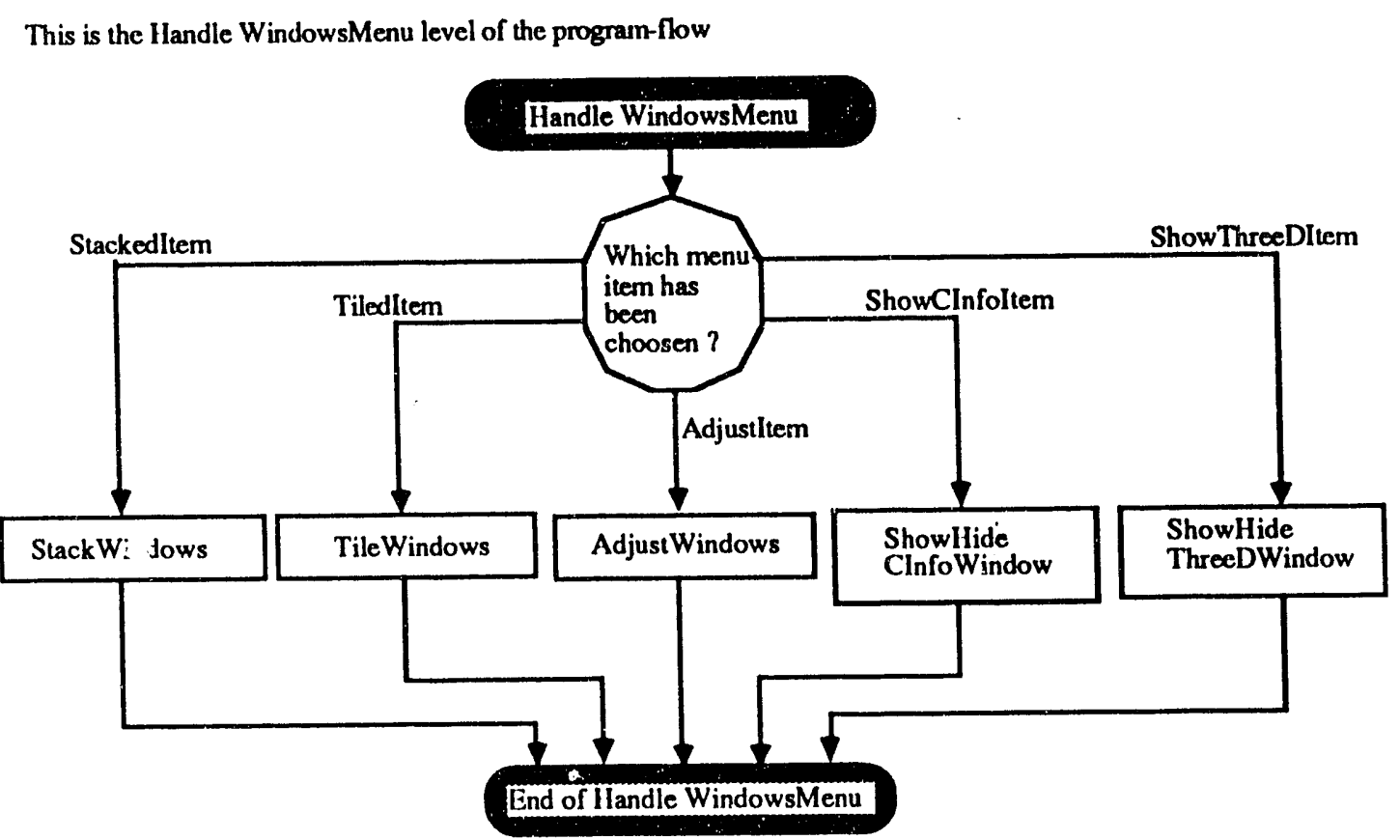




\section{HandleThreeDMenu}

This is the 'Handle ThreeDMenu' level of the program-flow

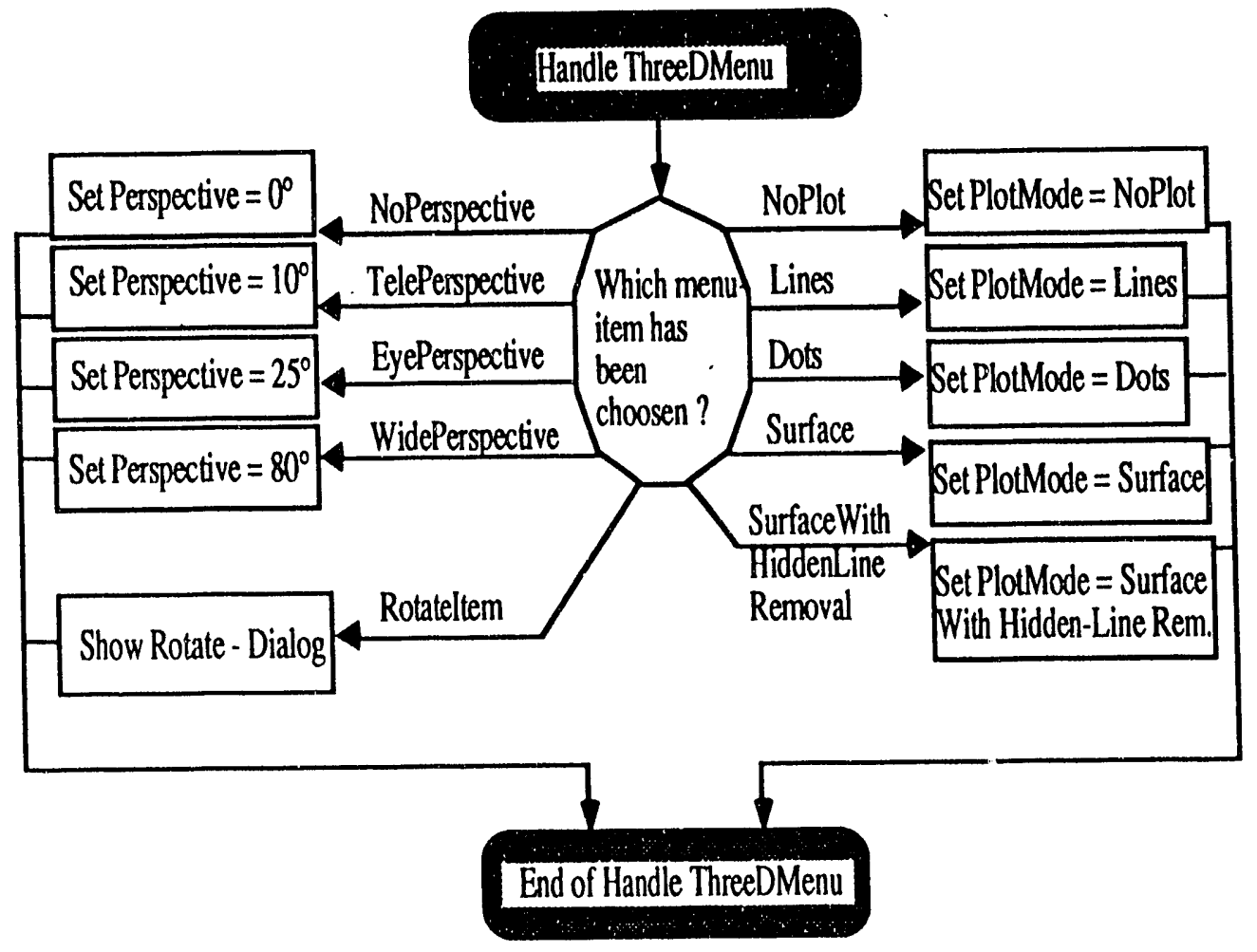




\section{MouseInContent}

This is the DoMouselnContent level of the program-flow

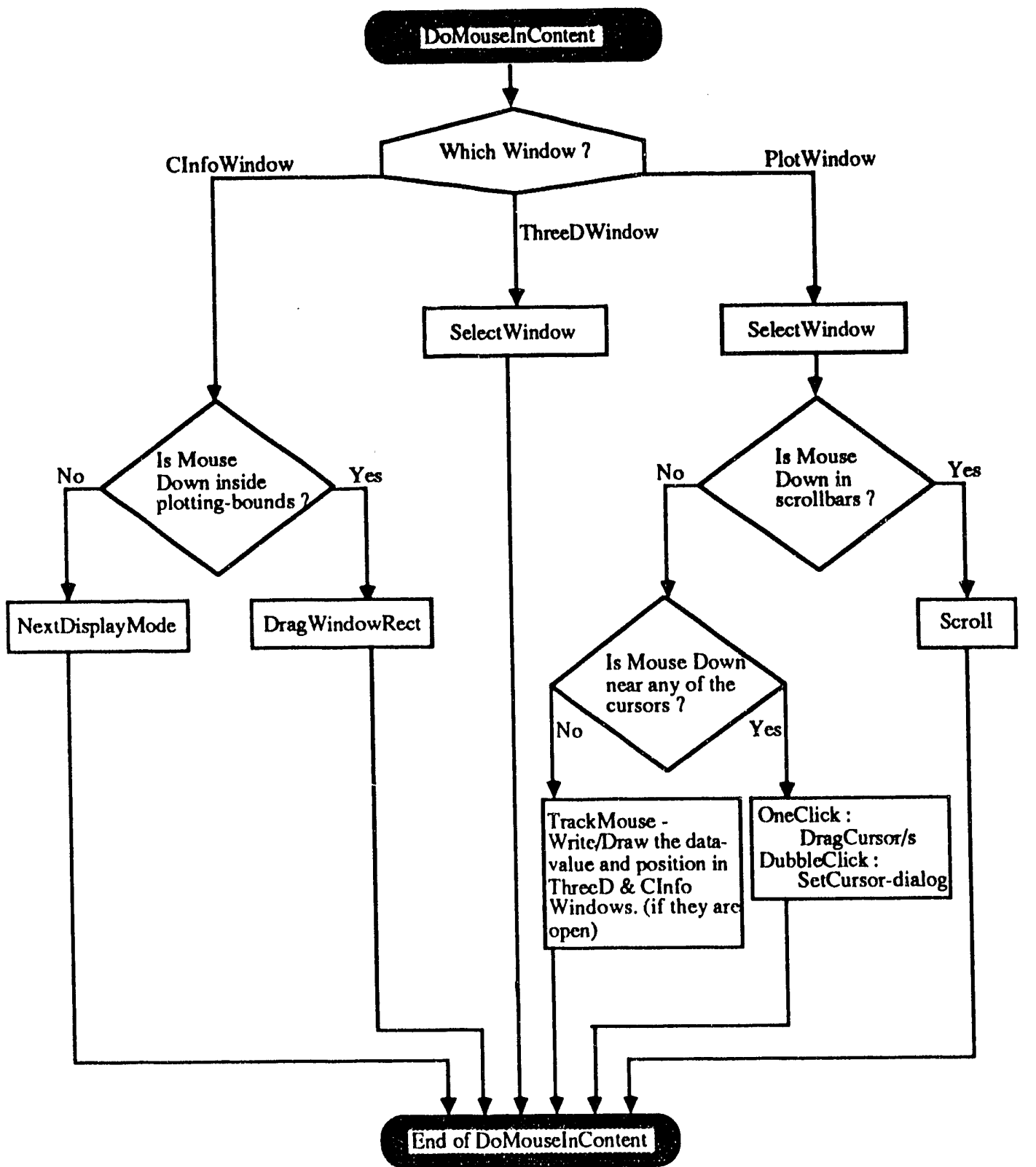




\section{DealWithKeyDowns}

This is the DealWithKeyDowns level of the program-flow

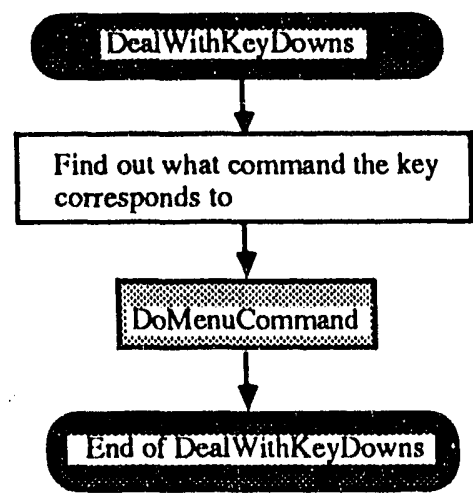

\section{DealWithActivates}

This is the DealWithActivates level of the program-flow

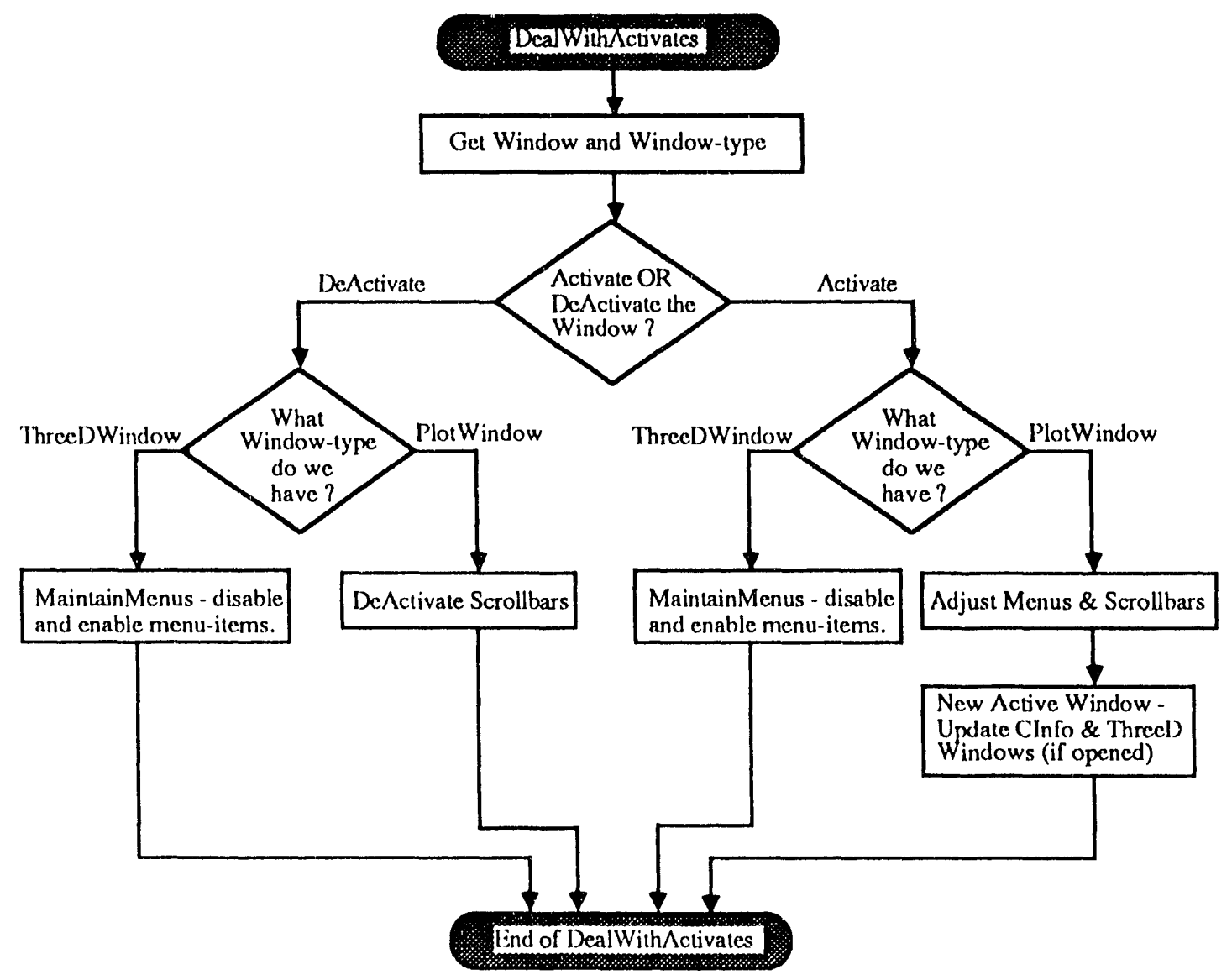




\section{DealWithUpdates}

This is the DealWithUpdates level of the program-flow

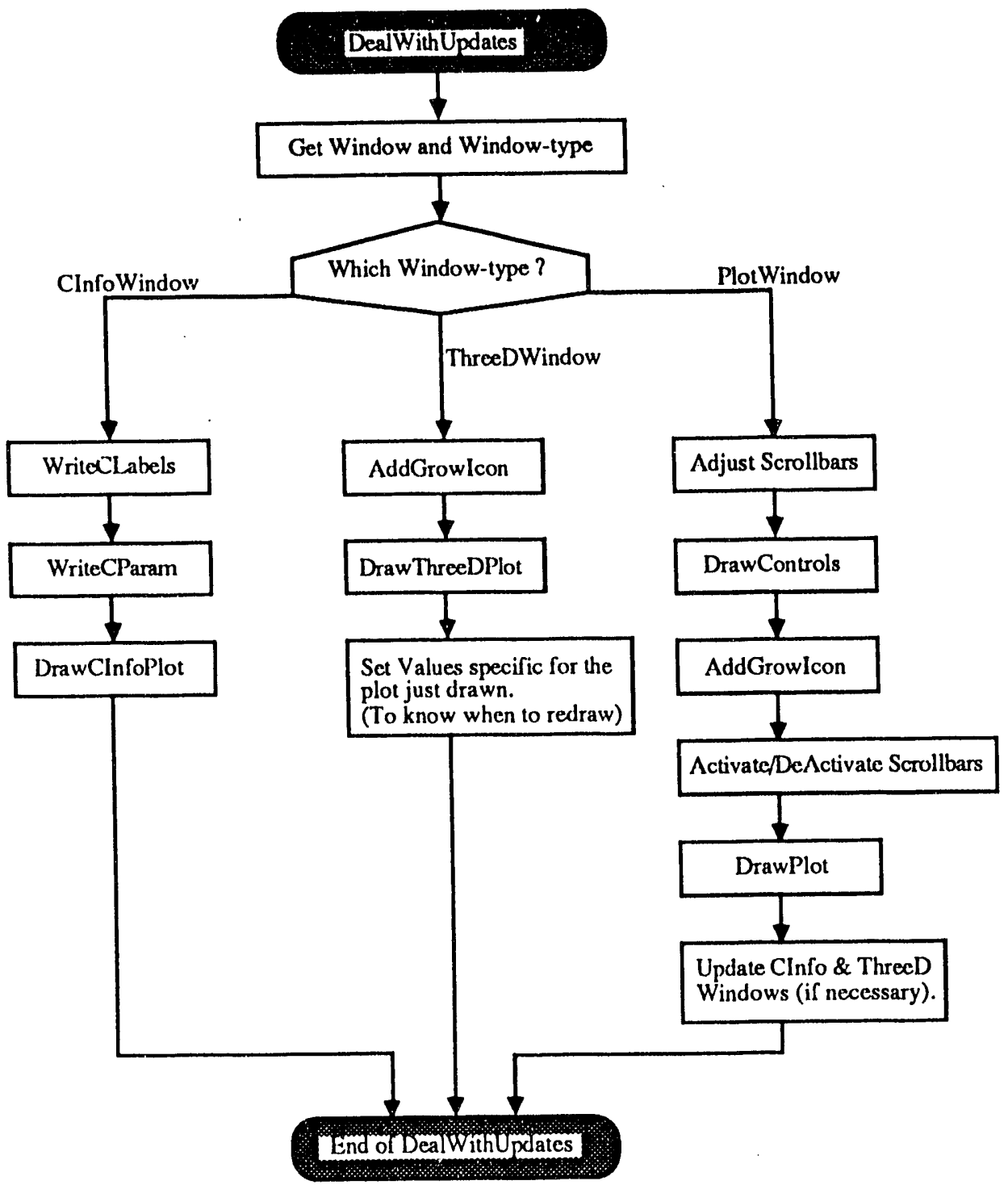




\title{
Appendix B : Photographs
}

\begin{abstract}
About the photos
The idea of taking pictures of the screen came from the fact that it would be a nice way of showing people the plots without having to drag them to the screen of a Macintosh. This is how we did it: We waited until it was dark outside - then we turned the light off in the room, we did this to minimize the reflections of light on the screen. The camera was attached to the tripod and when shooting I used the 'program-mode' and the self-timer. The self-timer was used to prevent vibrations since the exposure time was $1 / 30$ of a second. The zoom-lens was set to $50 \mathrm{~mm}$ to prevent distortions. No flash was used. We used 100 ASA film.

This is what the different photos are showing:

Photo 1: This photo shows gamma-ray data with an angle of 90 degrees between the gamma-ray source and the gamma-ray detector. The data is compressed 6 times $(360 \times 180 / 6=60 \times 30)$ to get a nice $3-D$ plot.

Photo 2: This photo shows the same data as above but now we are pulling down the plot-menu in order to chose the 'Expand'-command.

Photo 3: Now we have expanded the area limited by the cursors. What we see is the density plot of the cursor-area and also the perspective (3-D) plot of the same area.

the

Photo 4: This photo shows the 90 degree data and lets you see the structure in middle of the data-set, at the bottom of the 'bowl'.
\end{abstract}




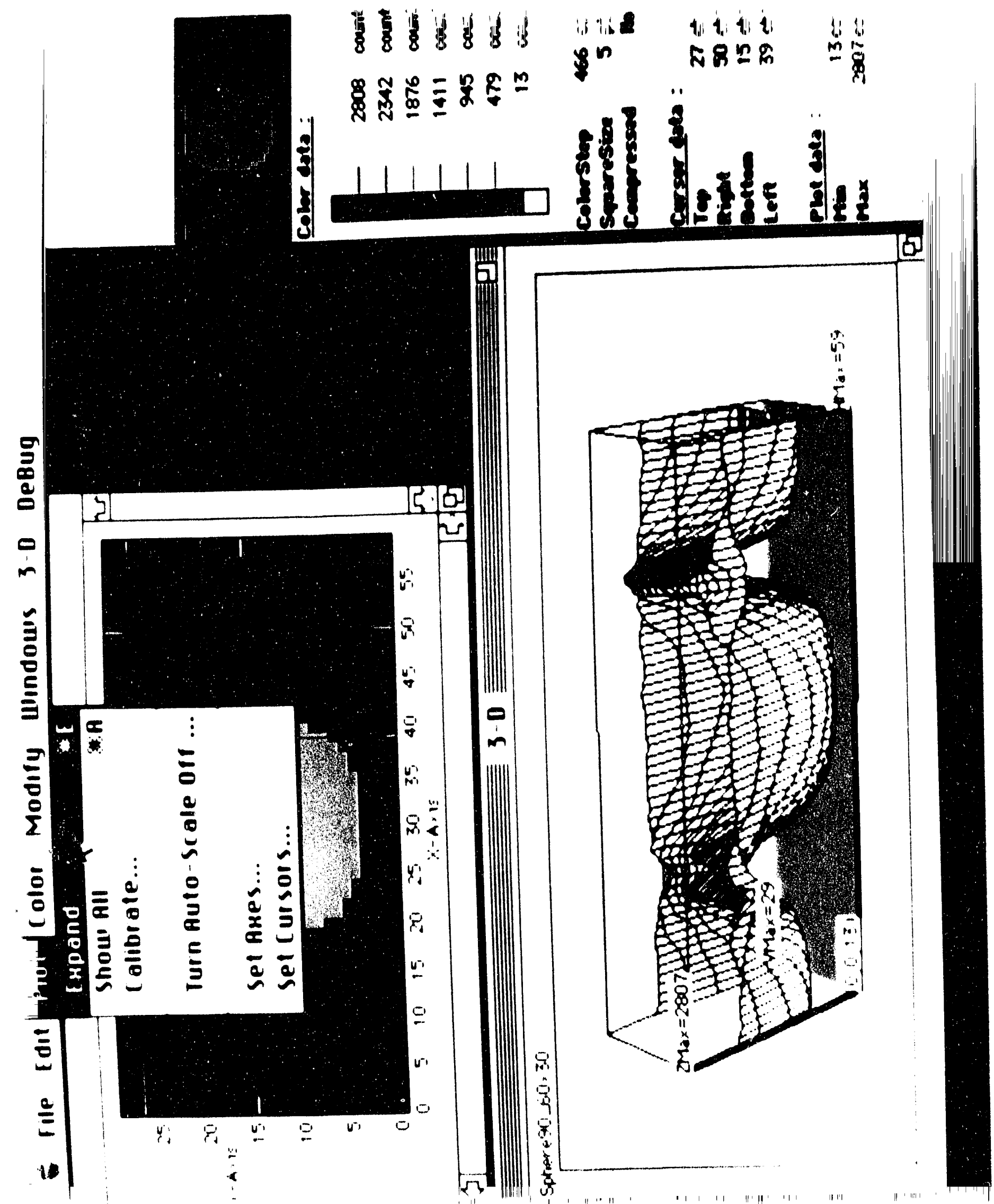




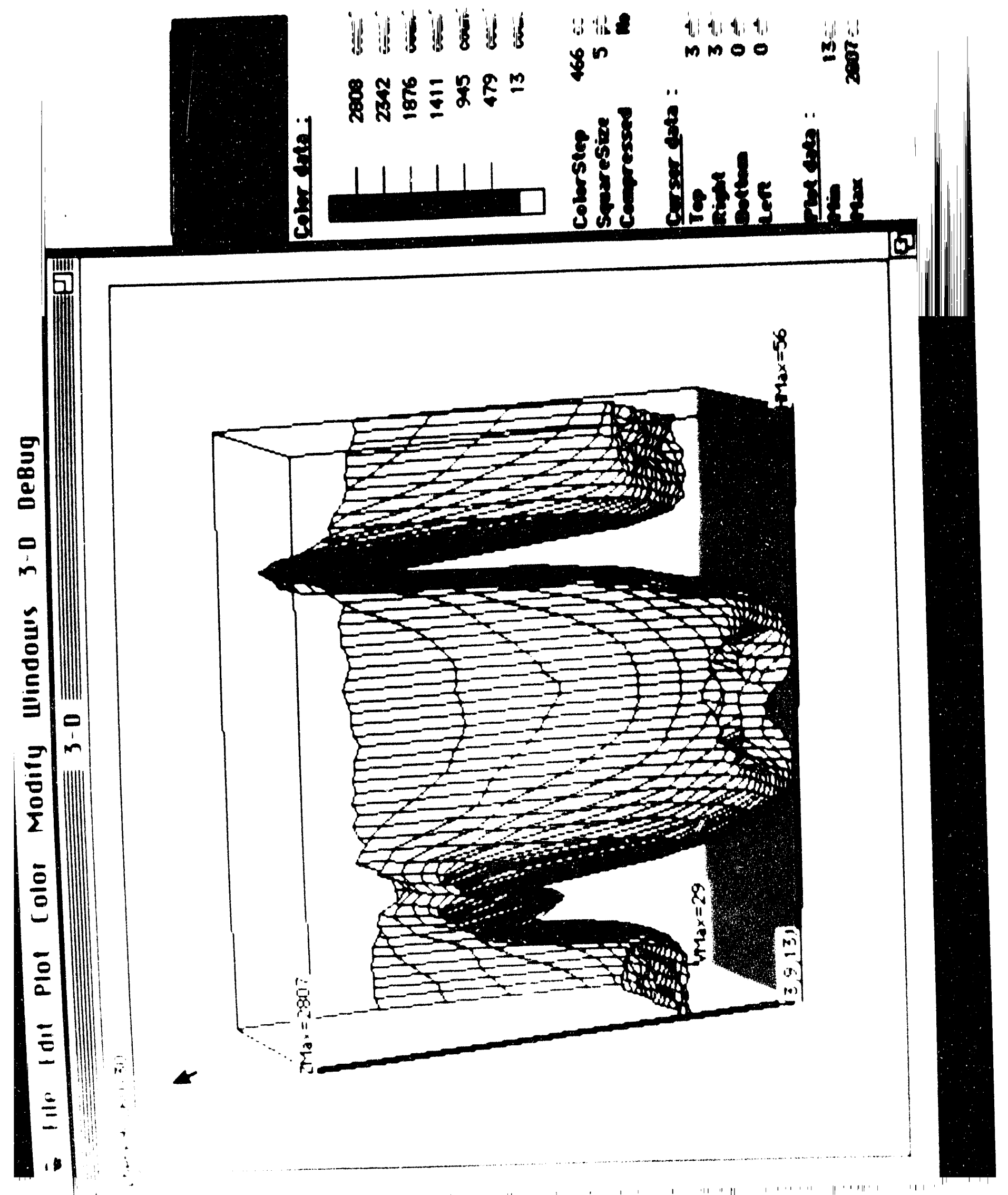




\title{
Appendix-B Student Report, Peter Agnvall
}

\author{
A FAST FOCAL PLANE DETECTOR \\ FOR THE FRAGMENT MASS ANALYZER AT ATLAS
}

$* * * * * * \mathrm{DRAFT} * * * * * *$

\author{
Peter Agnvall \\ Royal Institute of Technology \\ Stockholm, Sweden \\ University of Florida \\ Gainesville, Florida
}

January, 1989 


\section{ACKNOWLEDGEMENTS}

I am by this report going to describe and explain a master thesis work done by me, Peter Agnvall, at the Space Astronomy Laboratory of the University of Florida.

I am a student in electrical engineering at the Royal Institute of Technology, Stockholm.

My major is in physical electrical engineering. When it was time for the master thesis work, I had the great opportunity to come to the University of Florida, thanks to Professor Rune Lindgren, (Department of Aerospace Engineering, Mechanics \& Engineering Science ) and the Royal Institute of Technology, to do it.

I would also thank the staff at Space Astronomy Laboratory and especially those I have been working with:

- Dr. R.B. Piercey

- Dr. M.L. Muga

- Dr. Penny Haskins

- Jack McKisson

- Drew Weisenberger

- Don Ely 
CONTENTS

I. INTRODUCTION 4

II FRAGMENT MASS ANALYZER AT ATLAS 4

III THIN FILM DETECTOR

1. INSRUMENT DESIGN

3. PLASTIC FILM 10

IV DATA ACQUISITION SYSTEM

1. KMAX 11

2. HOW TO USE KMAX 12

$\checkmark$ DETECTOR PERFORMANCE 13

VI - THE STOPI PROGRAM

1. THEORY 14

2. THE PROGRAM 14

3. FLOWCHARTS 15

4. RUN EXEMPLE 17

VII - MEASUREMENTS.

1. TEST FIXTURE 21

2. THE ABSOLUTLY FIRST MEASUREMENT. 23

3. THE SECOND MEASUREMENT. 23

4. THE THIRD MEASUREMENT. 24

VIII- FUTURE TESTS.

IX-CONCLUTIONS. 


\title{
INTRODUCTION
}

\author{
WHAT IS IS \\ HOW IT IS USED (SECONDARY BEAMS ETC) \\ WHAT IT WILL DO \\ WHAT WE WILL REPORT IN THIS THESIS
}

FRAGMENT MASS ANALYZER AT ATLAS

At Argonne National Laboratory (University of Chicago), a large acceptance Fragment Mass Analyzer is under construction ${ }^{1}$. This device will be used to separate nuclear reaction products from the beam and transport them to a detector station at the focal plane. The solid angle of the FNA will be exceptionally large, and a large range of energies and masses will be simultaneously accepted. Primary ion beams are produced by a tandem Van de Graaff coupled to a linear accelerator. Ions injected into the FMA and transported to the focal plane will be dispersed in $M / Q$ (mass/charge), with a resolution of better than $1 / 300$. The primary beam is expected to be rejected by approximately $10^{-12}$. Such excellent characteristics are expected to provide new and exciting opportunities for a number of different experimental programs.

Since the ions will be dispersed in $M / Q$, measurements ot position, energy and energy loss will enable the nucleon number $A$ and proton number $Z$ of the particles to be determined. In addition to good mass resolution, it is desirable for a mass spectrometer operating on the heavy ions emerging from nuclear reactions to have large acceptance in energy, mass and angle. For fusion recoils, the angular distribution is usually peaked at zero degrees, making good separation from the beam mandatory for observing fusion products.

The large energy acceptance places restrictions on the amol'nt of chromatic aberration at the focus, requiring that the optics of the device be studied to high order. This also implies that strong quadrupole focussing should be avoided if possible, because of the chromatic aberrations that these lenses introduce. In order to provide mass information on the wide range of ions emerging as nuclear reaction products, a combination of electric and magnetic fields is required. A system using only magnetic fields can, in principle, provide very high

${ }^{1}$ Argonne National L aboratory, University of Chicago, A Proposal For A Fragment Mass Analyzer At ATLAS,(1986). 
mass resolution, but it will have a very narrow energy acceptance. In certain cases, limitations, primarily on the attainable electric fields, restrict the energies and masses of ions that can be accommodated by the FMA. As previously stated, the FMA disperses in $\mathrm{M} / \mathrm{Q}$, and therefore will rely on measurements of energy and energy loss at the focal plane to determine $\mathrm{Q}$ and the atomic number $\mathrm{Z}$ of the ions.

The versatility of the FMA permits it to be used in a variety of scientific applications:

- It's high transmission makes it an ideal tool to search for superheavy elements.

- Both neutron-deficient an neutron-rich nuclei far from stability can be produced and separated in numbers sufficient to enable their masses to be measured.

- With the FMA serving as a mass filter in coincidence with detectors at the target position, an entirely new class of experiments becomes possible, aimed at the study of weak nuclear reaction channels.

- The FMA can be effectively used in the investigation of many types of nuclear reactions, including quasi-elastic scattering and transfer reactions near the Coulomb barrier.

In many of the planned experimental programs it is very desirable to have additional information about the separated ions as they exit the device at the focal plane of the detector. This additional information might include measurements of the time-of-flight, nuclear $\mathrm{Z}$, energy loss characteristics, etc.

\section{THIN FILM DETECTOR}

The Space Astronomy Laboratory is designing, constructing and testing a dual film, focal plane detector to be used as a fast event trigger, $\Delta \mathrm{E}$ and time of flight spectrometer for the Argonne Fragment Mass Analyzer.

In the past, the most common focal-plane detector systems used in conjunction with on-line recoil mass spectrometers have been solid state surface barrier detectors and gas counters. Experiments which require some nuclear $Z$ information have normally used $\Delta E-E$ telescope counters ${ }^{1}$. With these detectors, $Z$ determination is based on the observation that,

${ }^{1}$ Goulding, F.S. and Harvey, B.G., Ann. Rev. Nucl.Sci., 25, 167(1975). 
for a given ion energy, the partial energy-loss, $\Delta \mathrm{E}$, measured with a transmission detector depends only on the nuclear charge $\mathrm{Z}$ and the mass number $\mathrm{A}$ of the ion.

For the most part, solid state detectors have been used for these $\Delta \mathrm{E}$ measurement. Although they have a good energy resolution, it is often difficult to manufacture very thin detectors since it would require near zero dead layers at both the particle entrance and exit windows. Generally, solid state $\Delta E$ detector thicknesses range from about $1 \mathrm{~g} / \mathrm{cm}^{2}$ to $1.0 \mathrm{mg} / \mathrm{cm}^{2}$ depending on the types and energies of ions being measured. The very thin detectors required for low-energy and/or heavy-ion measurements present serious problems in construction and handling of the thin wafers. Timing with solid state detectors depends on many factors but is normally no better than a few nanoseconds to a few tens of nanoseconds and can be worse for detectors which have been optimized for energy resolution. Furthermore, the performance of solid state detectors are known to degrade after total fluxes of $10^{7}-10^{8}$ particles. This makes them unsuitable for high rate applications such as high background measurements and secondary beams experiments. Since the thinnest available silicon $\Delta \mathrm{E}$ detectors absorb to much energy for many applications, attention was placed early on gas ionization $\triangle \mathrm{E}$ detectors for heavy ion identification. Gas $\Delta E$ proportional counters, while not offering the energy resolution of silicon $\Delta \mathrm{E}$ detectors, can be made much thinner. The intricacies of gas handling required to maintain constant low pressures is a drawback, however, and usually limits their use when space is limited or vacuum requirements are severe. The slower charge collection over the larger distances of the gaseous $\Delta \mathrm{E}$ counter results in poor timing characteristics. This prevents their use in experiments where high counting rates are expected or in applications requiring fast timing signals for coincidence or time-of-flight measurements.

Based on research done over the past few years at the University of Florida 1,2,3, a detector system is proposed, which addresses many of the limitations discussed above. This design incorporates two thin film detector modules, each of which can provide a fast timing trigger and a pulse height related to the energy loss in the film. Accurate velocities can then be determined from the time differences and energies can be calculated from the ion mass. The two $\Delta \mathrm{E}$ values can be used alone or in conjunction with other focal plane devices to determine the $\mathrm{Z}$ of the transiting ion. Furthermore, the thin film detector can preform without degradation at very high counting rates and survive total doses at least 100 times that of a solid state detector.

\footnotetext{
${ }^{1}$ Muga, M.L. and Griffith, G.L.,Phys.Rev., B, 2, 3639(1974)

${ }^{2}$ Nicovich, J.M., Clem, A., Muga, L. and Plendl, H.S., Nucl. Instr. Meth. 157, 93(1978).

${ }^{3}$ Muga, M.L. and Griffith, G.L., Nucl. Instr. Meth. 109, 289(1973).
} 
Target

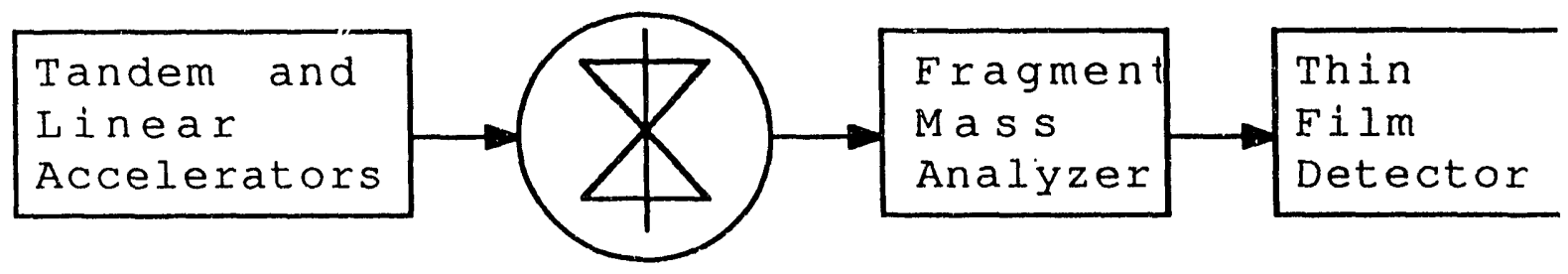

Figure 1. Schematic layout of Argonne accelerator.

\section{INSTRUMENT DESIGN}

The principle of the detector is illustrated in figure 2. As the reaction products exit the FMA they will pass two identical Thin Film Detectors. The module consist of a thin scintillator film mounted on a frame and viewed by two large photomultiplier tubes. In each module, the pulse of light which results from the passage through the film is collected by the photomultiplier tubes ${ }^{1}$. By using large tubes, the solid angle for light collection can be made adequate without limiting the beam path. Each of the modules will provide a time trigger and a pulse height associated with the transit.
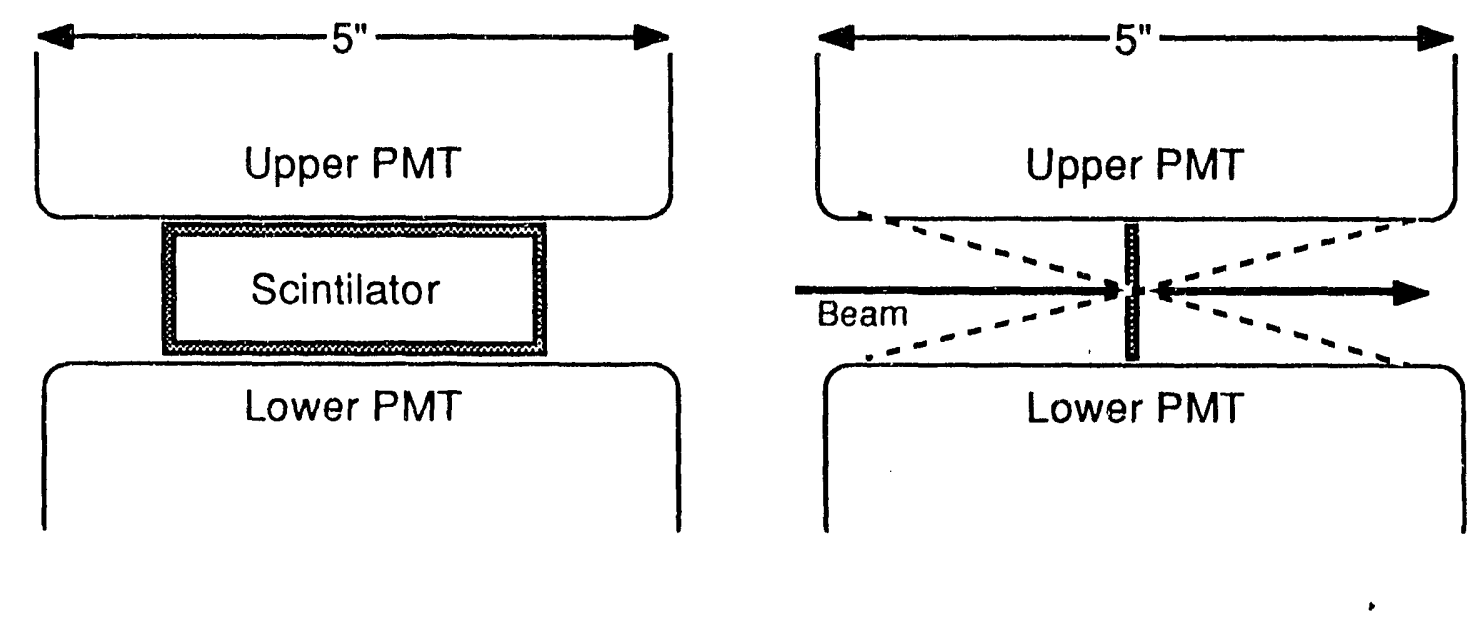

\footnotetext{
${ }^{1}$ R.B.Picrey, Space Astronomy L_aboratory, University of Florida, A Fast Focal-Plane Detector, (1988)
} 
View perpendicular to beam path.

View along beam path.

Figure 2. Cross section of the PMT-scintillator geometry

Figure 2 illustrates how each of the photomultiplier tubes collects light from the thin rectangular scintillator film. Due to mass dispersion, achromatic focussing and geometrical collimation the reaction products reach the focal plane of the FMA, distributed over a rectangular area roughly 2.5 by $6 \mathrm{~cm}$. The first test films are manufactured roughly 1 inch by 3 inches in order to cover this entire area. Since the photomultiplier tubes are somewhat larger than the films, edge effects should be minor and reasonable linearity over the entire foil can be achieved.

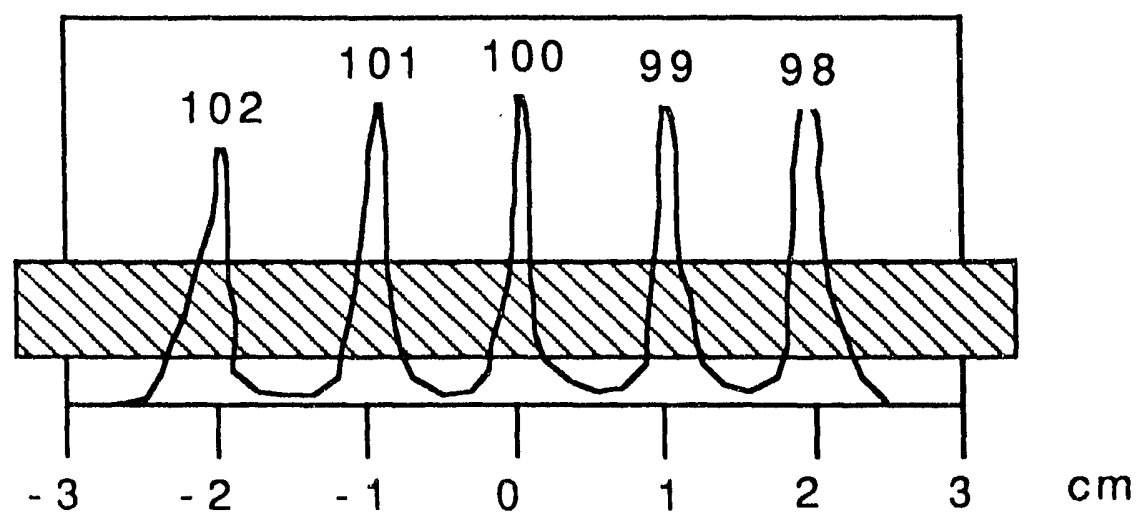



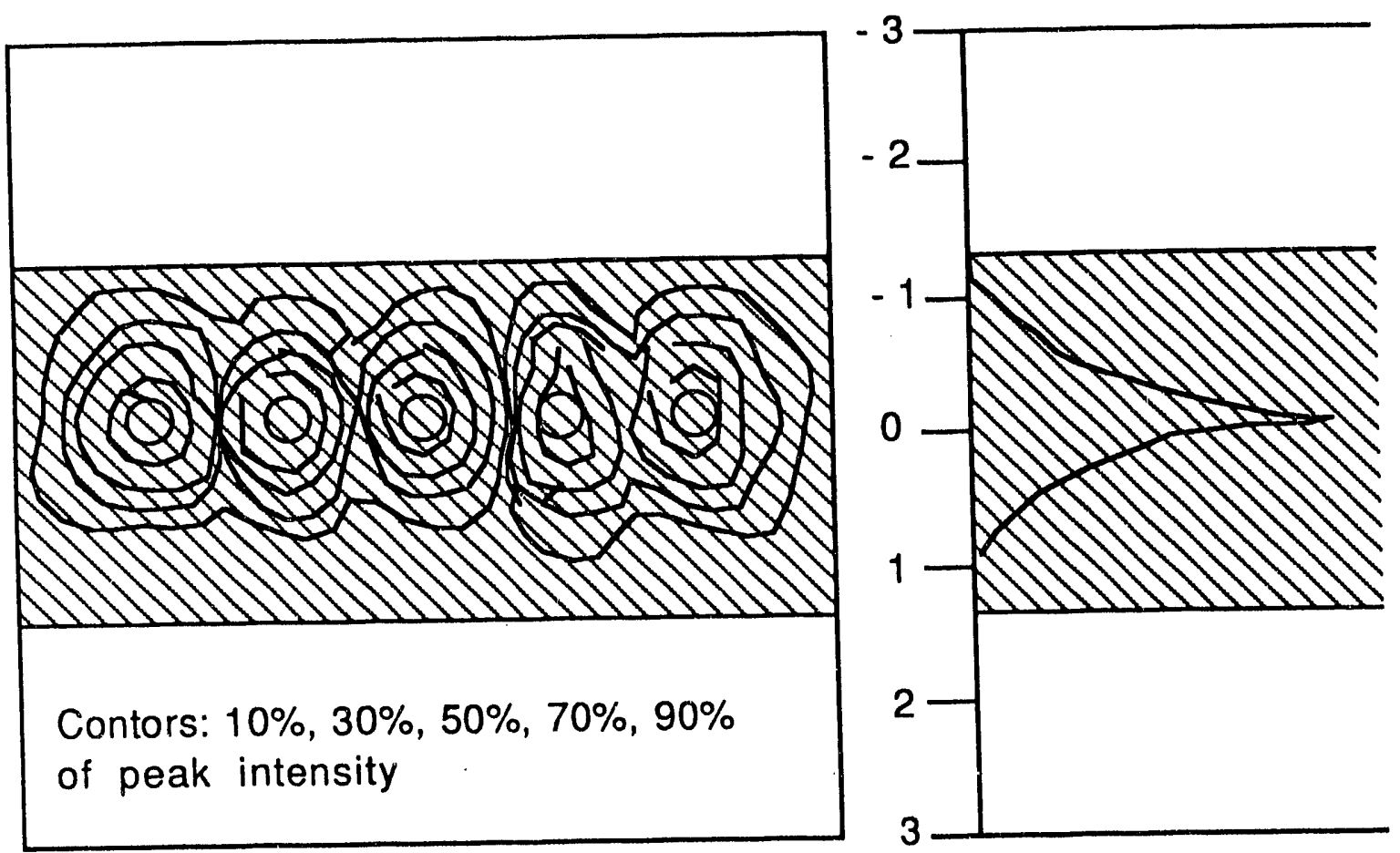

Figure 3. Portion of the focal plane covered by the proposed thin film detector.

Figure 3 shows the size of the 1 by 3 inch film relative to the predicted structure of a typical distribution of reaction products at the focal plane of the FMA. From the figure it is clear that the film size will not limit the useful area of the FMA focal plane, and that the thin film detector should be compatible with other focal plane detectors.

For each module the photomultiplier tubes and thin film is installed in a 6-way vacuum fixture. Figure 4 shows three perpendicular views of the proposed model design. Figure $4 a$ shows the view along the "film insertion axis", figure $4 \mathrm{~b}$ shows the view along the "beam axis" and figure $4 c$ shows the view along the "photomultiplier tubes axis". Beam collimators and/or any necessary light baffles are not shown and may be particular to the reaction being studied. 


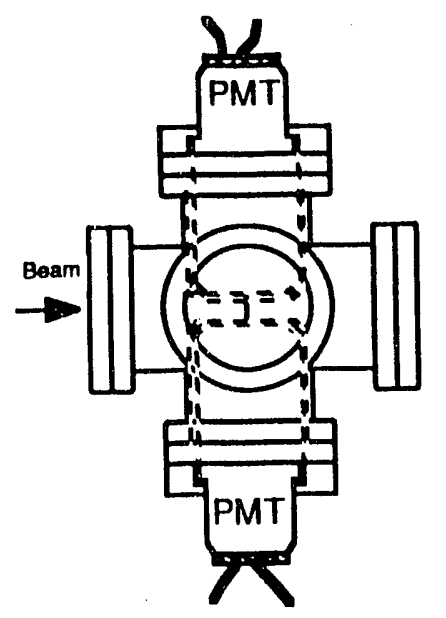

$4 a$

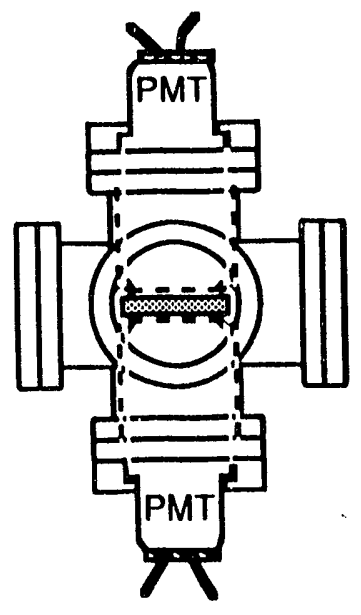

$4 b$

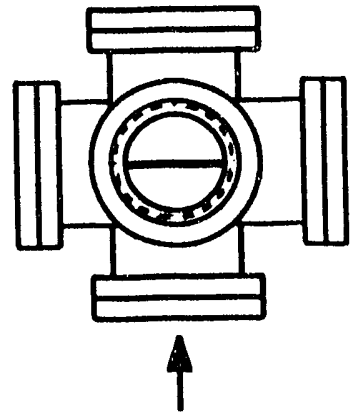

$4 c$

Figure 4. Three perpendicular views of the 6-way detector chamber. 


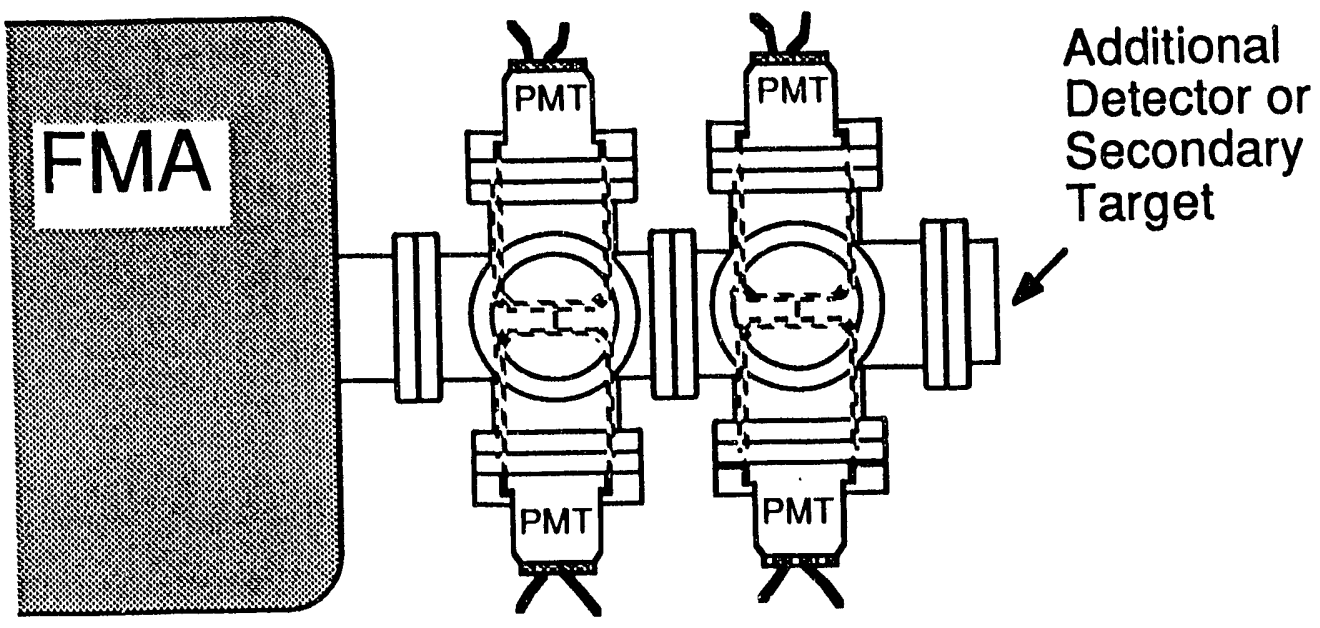

Figure 5. The proposed dual film detector attached to the FMA.

In figure 5 a concept drawing of how the dual thin film detector system might look mounted at the focal plane of the FMA. Additional detectors specific to the particular experiment are not shown in the figure and would be supplied by the experimenter.

Also, in order to get proper alignment, a flexible joint between the FMA and the first thin film detector module should be considered.

\section{PLASTIC FILM}

The plastic film we used was a mixture of polyvinyltoluene and p-terphenyl, which is the prime scintillator. The proportions are $98 \%$ polyvinyltoluene and $2 \%$ p-terphenyl. Figure 7 shows the molecular structure of these two plastics.

Polyvinyltoluene

P-terphenyl 

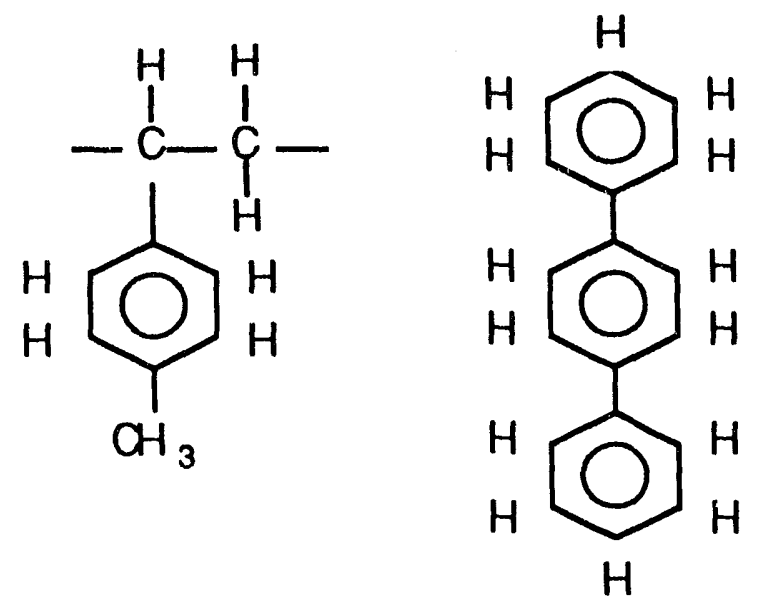

$$
\mathrm{C}_{9} \mathrm{H}_{10} \quad \mathrm{C}_{18} \mathrm{H}_{14}
$$

Figure 6. Polyvinyltoluene and P-terphenyl witch the plastic film is made of.

The plastic film was made at the Department of Chemistry, University of Florida. The plastic scintillators are added to a solution made of ethyl acetate and amyl acetate. The mixture is allowed to stand with occasional stirring until complete dissolution occurs and then transferred to a container with a drop spout. A photographic developing pan is filled with distilled water at room temperature. A glass plate is partly immersed at one end of the pan and inclined at an angle of 60 degrees with the surface of the water. Two or three drops of the film solution are squeezed out onto the center of the glass plate just above the water line. As the solution contacts the water, it flows out over the water and a solid film appears on the surface. The solvent is absorbed by the water and then the film is lifted of the water and put on a frame. The film is now very thin, which means that the energy loss and the light output in the film is small. If a higher light output is needed, several films can be laminated, with up to 50 laminations ${ }^{1}$.

\section{DATA ACQUISITION SYSTEM}

\section{KMAX}

Kmax is a multifunction data acquisition and control interface for the Macintosh II computer in a CAMAC hardware environment. With Kmax the user has control of how the data is taken, how it is sorted, how it is recorded and how it is displayed.

\footnotetext{
${ }^{1}$ Muga, M.L., Burnsed, D.J. and Stecger, W.E., Nucl. Inst. Meth. ,104, 605(1972).
} 
Since Kmax supports CAMAC, the user is not locked into any special data acquisition/control hardware. The optimized Kmax software leads the user through each step in defining a data acquisition setup with any set of CAMAC modules.

\section{HOW TO USE KMAX}

First the user defines the Crate setup by "installing" the necessary modules into a symbolic representation of the CAMAC crate, "dragging" them with the mouse to the appropriate slots. Thereafter, Kmax will utilize Module Description Resources to lead the user through definition of sequences made up of data acquisition and crate control operations. At each step, detailed parameters and descriptions of the modules "installed" in the crate are used to guide the user. In the Kmax architecture, data is acquired event-by-event in response to control events generated in hardware and/or in software. The data acquired in that event may consist of a number of loosely related parameters or it may be a block of data ( a histogram, for example).

Lists of instructions called Command Sequences control the interaction between the Macintosh and the CAMAC hardware. There can be several command sequences active at any time, each of which is executed in response to a particular event type. An initialization (GO) sequence, for example, is executed each time the GO button is clicked by the user. A service request (SRQ) sequence is executed each time the GPIB issues an SRQ. The command sequences are configured by the user for his particular application and may be modified at any time.

The data, read from the modules or otherwise generated by command sequences, is formatted as an event record and entered into the event data stream. The data stream may be sorted in real time into a variety of plot data structures or sorted as Event Data Files for later replay and sorting. This method for data acquisition and sorting is often referred to as list mode or event-by-event data acquisition.

Sorting of the event data stream is accomplished by specifying the event type, parameter index, the plot length, the plot type, a data offset and a scale factor. Any one or more parameters in an event may be sorted and windows may be set on any of the parameters in an event to accomplish conditional sorting.

Once a create is setup, the sequences defined, the sorts specified and the formats of the Kmax histogram plot windows chosen, the entire setup can be saved as a Kmax Instrument File. The instrument file makes it easy to save and recall useful instruments so that the user may completely recreate the data acquisition setup at a later time. 


\section{DETECTOR PERFORMANCE}

At the University of Florida a number of measurements, which can be extrapolated to predict the performance of the detector, has been made. Even though most of the measurements have been made with small area ultra thin films and small photomultipliers the result are already sufficient to justify the thin film detector. 


\section{VI - THE STOPI PROGRAM}

\section{THEORY}

For seventy-five years the stopping of energetic ions in matter has been a subject which has received great theoretical and experimental interest. The theoretical treatment has been done by Bohr, Bethe, Bloch and Lindhard. The stopping of ions in matter is defined as the mean energy loss of an ion passing through a thin amorphous elemental target. The term "stopping cross-section" is the energy loss per unit target thickness, usually expressed in units of $\mathrm{keV} /\left(\mathrm{mg} / \mathrm{cm}^{2}\right)$.

The total stopping cross-section of ions in matter is divided into two parts: the interaction of the ion with the target electrons, called electronic stopping, and with the target nuclei, called nuclear stopping. The nuclear stopping component can be separated because the heavy recoiling target nucleus can be considered to be unconnected to its lattice during the passage of the ion, and the interaction can be treated simply as the kinetic scattering of two screened particles. All the formulas in the STOPI program are taken from J.F. Zieglers book, Stopping Cross-Sections For Energetic Ions In All Elements (Pergamon press 1980). The STOPI program was written so we could compare the pulse height from the photomultipliers, which is related to the specific luminescence ( $\mathrm{dL} / \mathrm{dx}$ ), with the theoretical value of the energy loss, stopping power ( $\mathrm{dE} / \mathrm{dx}$ ), in the plastic film. The relation between specific luminescence and stopping power is

$$
\mathrm{dL} / \mathrm{dx}=\mathrm{A} \mathrm{dE} / \mathrm{dx} / 1+\mathrm{BdE} / \mathrm{dx}
$$

\section{THE PROGRAM}

The program was written on a Macintosh II in Pascal. One of the hard things was to create all the menus and windows, basicly the hole interface between user and machine. The Macintosh is known for it's user friendliness but it is not an easy machine to programme. The STOPI program is built up with by 9 modules:

- Stopl.r is the resource file. It contains window definitions, menu definitions and data tables, used in the calculation routines.

- StopITypes.p has all the type definitions.

- Fullinit.p initialize system routines used by the program. 
- GetUserInput.p creates a window and reads in the user input.

- Utilities.p reads in strings of data from resource file and makes numbers out of them.

- Calcul.p is the calculation routine.

- OutUnit.p converts numbers to strings, and takes care of the output.

- StopI.p is the main program.

- DebugUnit.p addresses the modem port, where a second screen is connected.

\section{FLOWCHARTS}

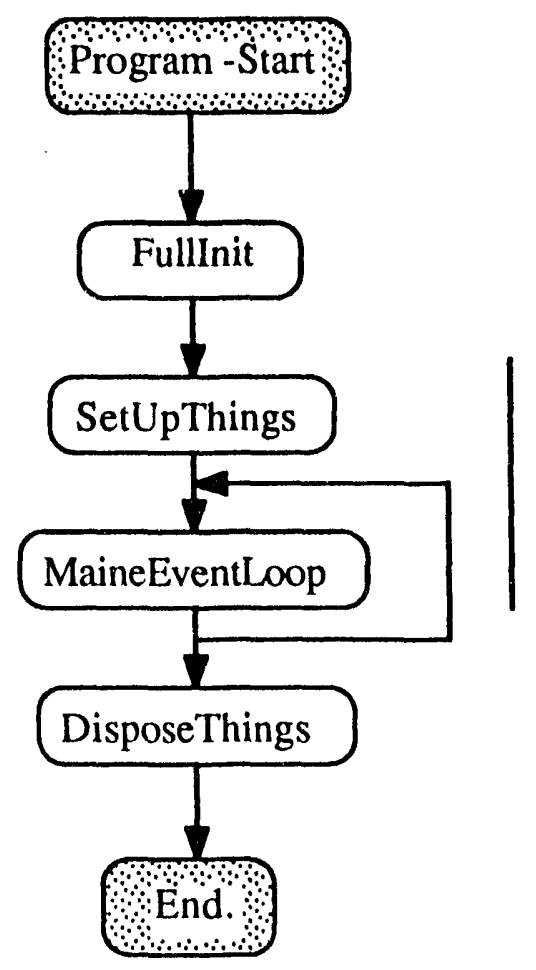

Figure 7. Flowchart over the main program unit. 


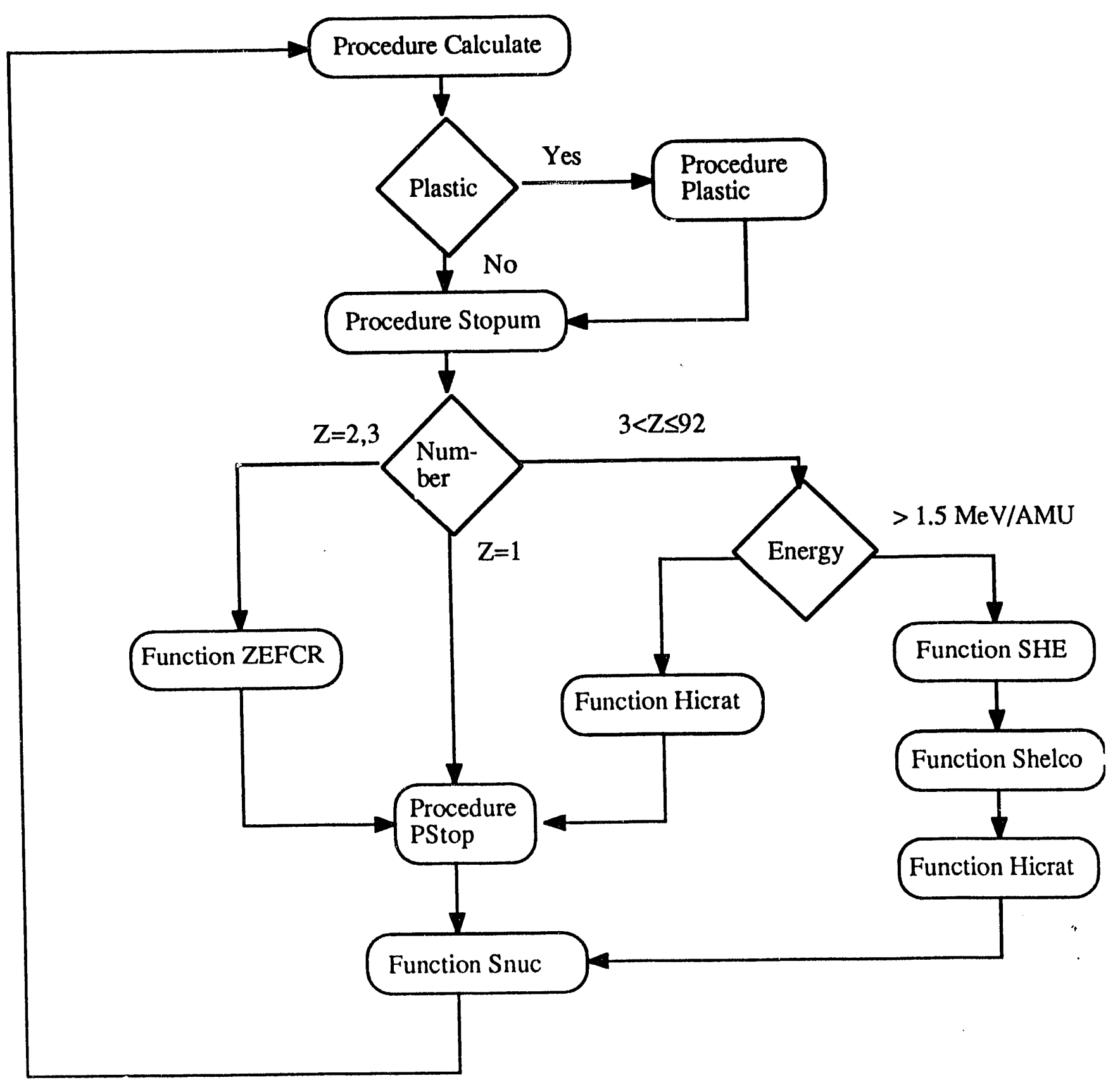

Figure 8. Flowchart over the calculation unit. 
For the stopping of ions in plastic, Procedure Plastic, the two individual stopping crosssections are added together and weighted by their relative abundance.

$$
\mathrm{S}(\text { compound })=\mathrm{aS}(\mathrm{A})+\mathrm{bS}(\mathrm{B})
$$

Where $\mathrm{CaHb}$ gives $\mathrm{a}$ and $\mathrm{b}$.

$$
98 * \mathrm{C} 9 \mathrm{H} 10 \text { and } 0.02 * \mathrm{C} 18 \mathrm{H} 14=\Rightarrow \mathrm{C} 9.18 \mathrm{H} 10.08
$$

Ratio $\mathrm{H} / \mathrm{C}=1.0980$.

Where $S(A)$ is the stopping cross-section with carbon as target at a certain energy and $S(B)$ is the stopping cross-section for hydrogen at the same energy.

\section{RUN EXEMPLE}

The following table shows the stopping powers for Nickel 58 as projectile and Carbon 12 as target. SNUC stands for the nuclear stopping. SE for the electronic stopping. SE is calculated by using an effective charge function (SE EFF-Q). Above 1.5 MeV/AMU a high energy formula is also used to calculate the electronic stopping(SE HE-FORM). STOT is the sum of SNUC and SE. In the last column, the ratio of SE EFF-Q and SE HE-FORM is calculated .

THIS IS THE STOPPING POWER FOR 58NI IONS IN 12C TARGET.

STOPPING POWERS CALCULATED.

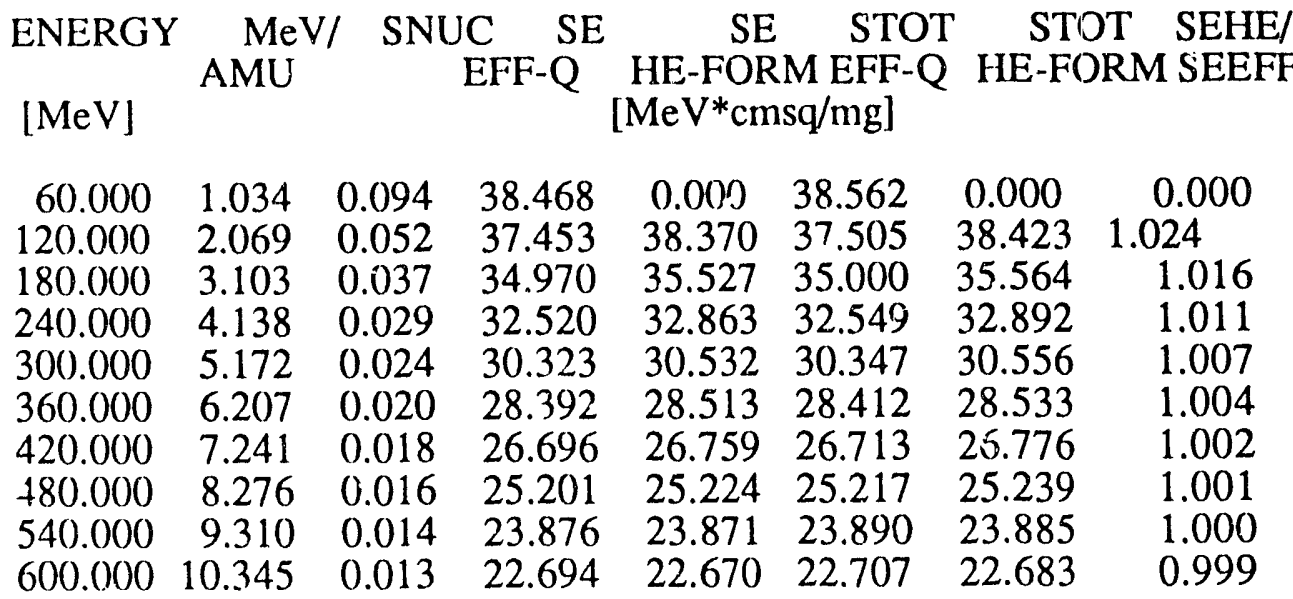




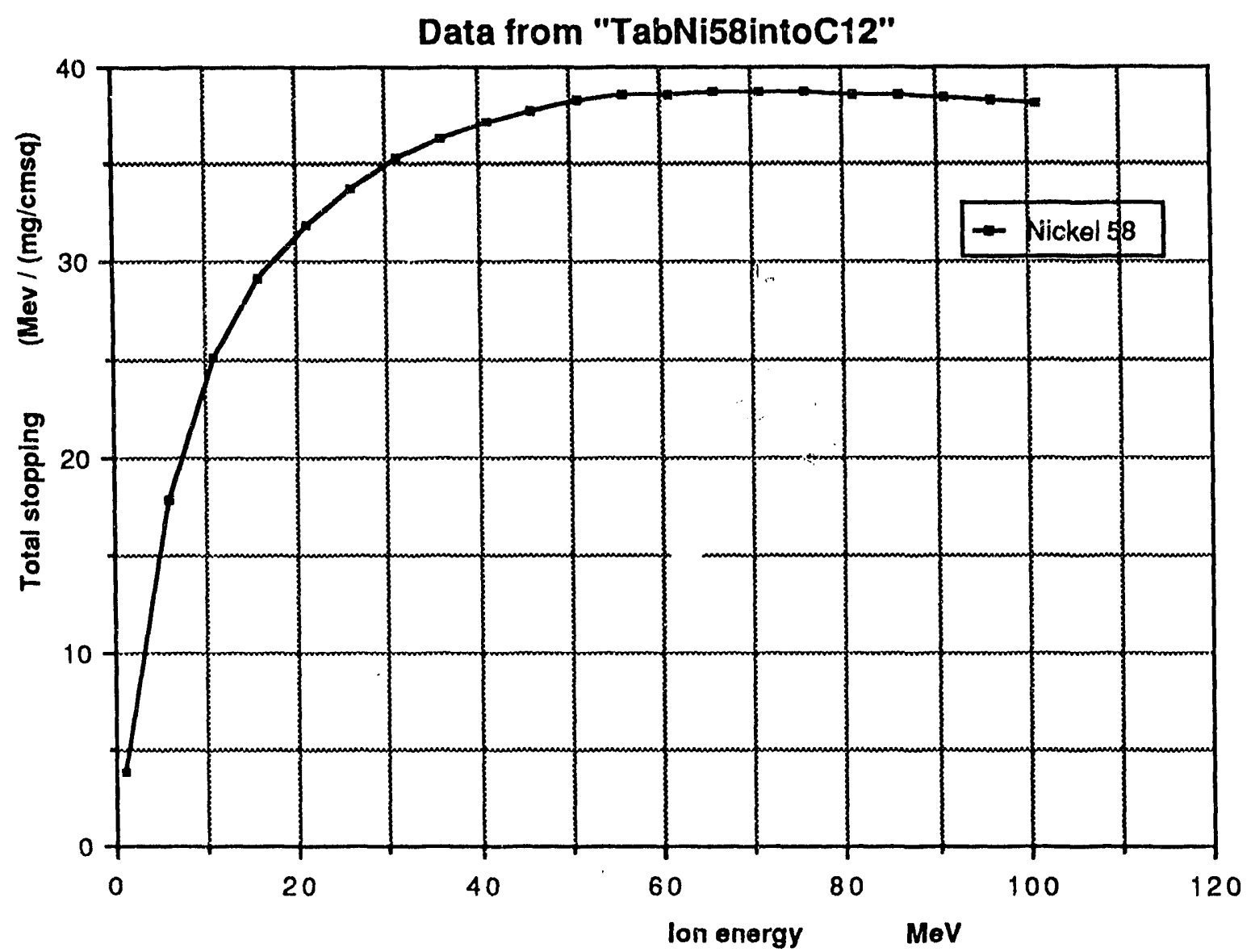

Figure 9. Shows stopping powers with Nickel 58 as projectile and Carbon 12 as target.

By using Cricket graph a graph of the stopping powers can be created. Here, we see energy as a function of STOT effective charge.The energy goes from $1 \mathrm{MeV}$ to $101 \mathrm{MeV}$. 


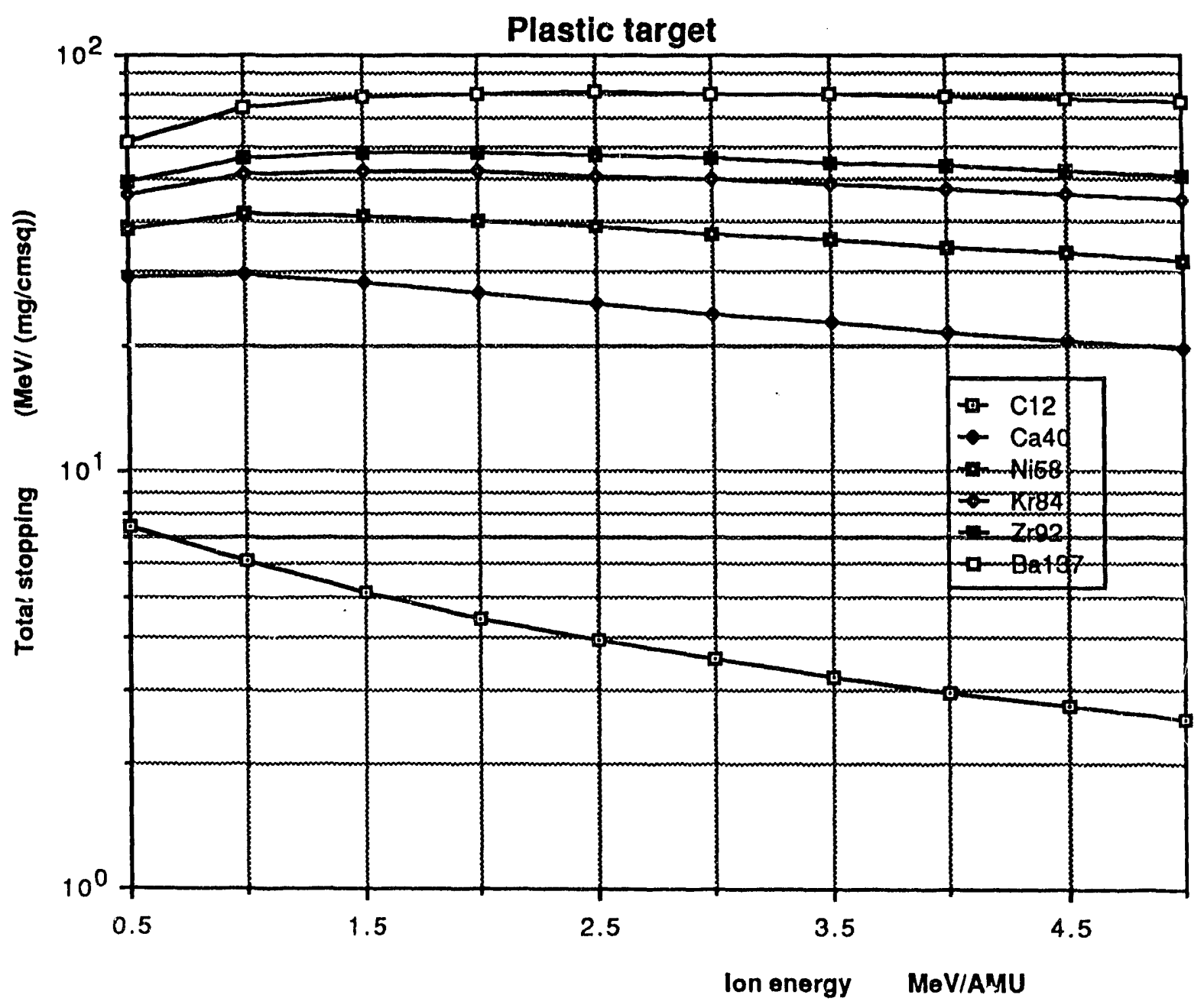

Figure 10. Shows stopping powers for different projectiles in a plastic target. 


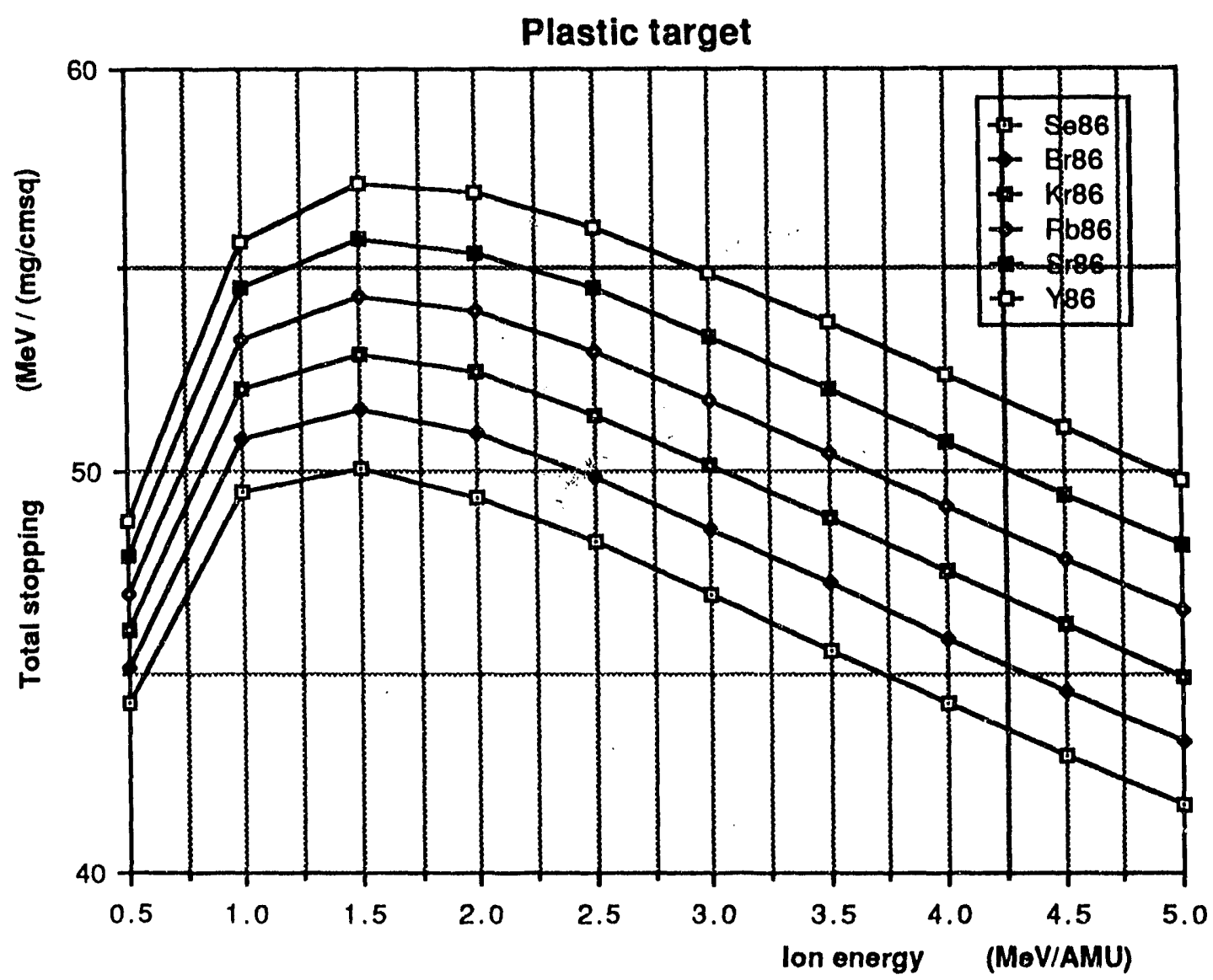

Figure 11. Shows stopping powers for different ions with the same nucleon number in a plastic target.

By using two Thin Film Detectors we can get the time of flight between the two detectors. Given the distance between the two plastic films, we can use the time of flight to find the particle velocity, thus the ion energy. As stated before, the pulse height from the photomultipliers is proportional to the stopping power. We can now go into the diagram and identify the particle. 


\section{VII - MEASUREMENTS.}

Photomultiplier tube : Vacuum pump : Power supply PMT Amplifier

Delay

Discriminators

Gate/Delay Generator

ADC

Controller

Computer
AMPEREX XP 2041.

Turbo molecular pump from General Electrics.

EG\&G ORTEC 556 HIGH VOLTAGE POWER SUPPLY.

: $\quad$ Model 777 PHILIPS SCIENTIFIC.

: $\quad$ EG\&G ORTEC 425A.

: $\quad$ Model 715 PHILIPS SCIENTIFIC

EG\&G ORTEC 584.

Model 794 PHILIPS SCIENTIFIC.

LeCroy 2249A.

: $\quad 3988$ GPIB CRATE CONTROLLER.

: Macintosh II.

\section{TEST FIXTURE}

The thin film detector chamber is tested on the test fixture illustrated in figure 5. The pump is a turbo molecular pump. The pressure is measured by an ionization gauge.

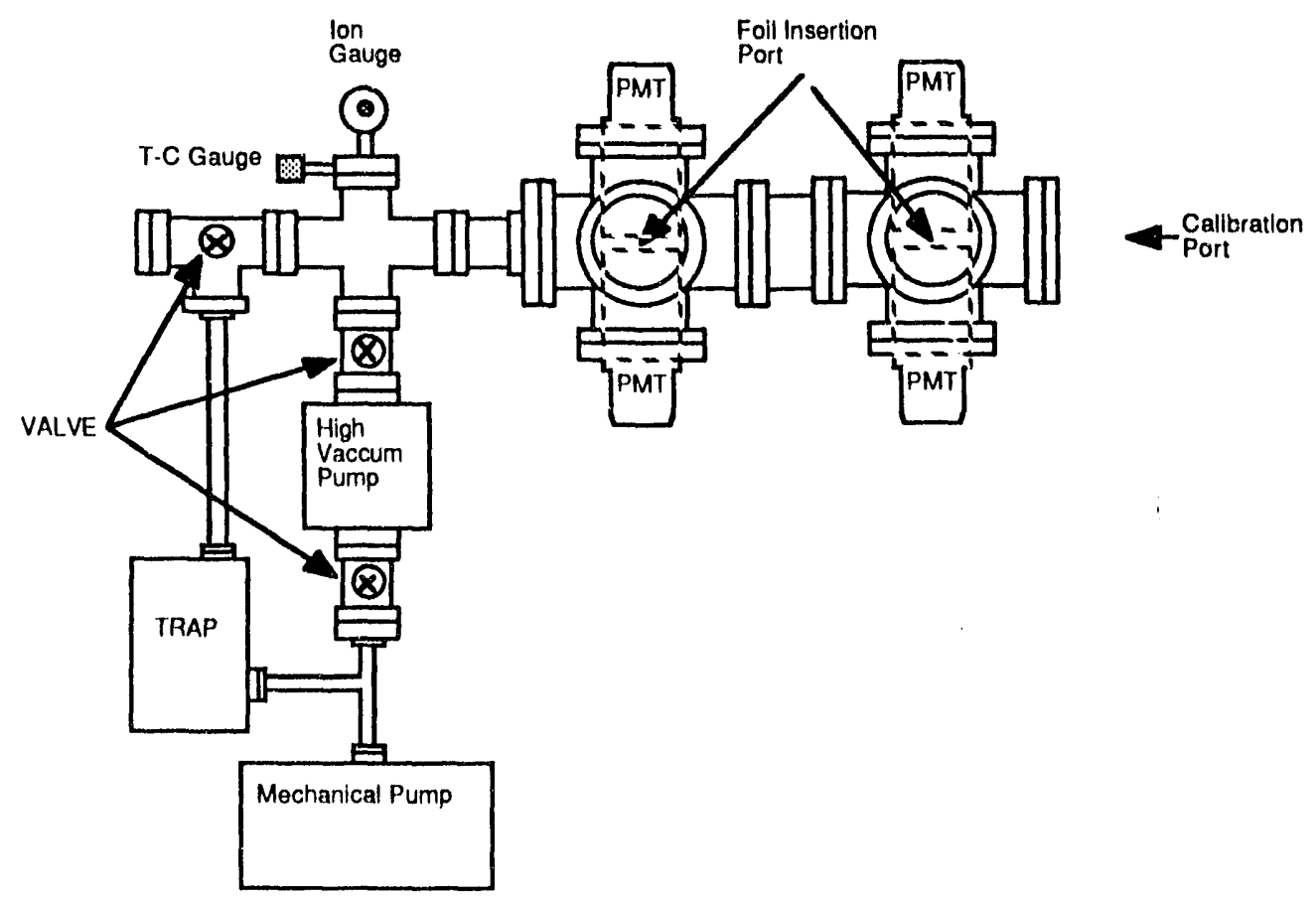

Figure 12. The test fixture for the thin film detectors. 
THE ABSOLUTLY FIRST MEASUREMENT.

In this experiment we looked at the background radiation with the lights on/lights off in the room. In the test fixture we used: one chamber and one PMT ( see figure 12 ). In the place for the other PMT and for the other chamber we putted covers so it would be light prof. Data were collected during ten minutes for each case. The system was not pumped down to vacuum. Power on PMT -2000 Volts.

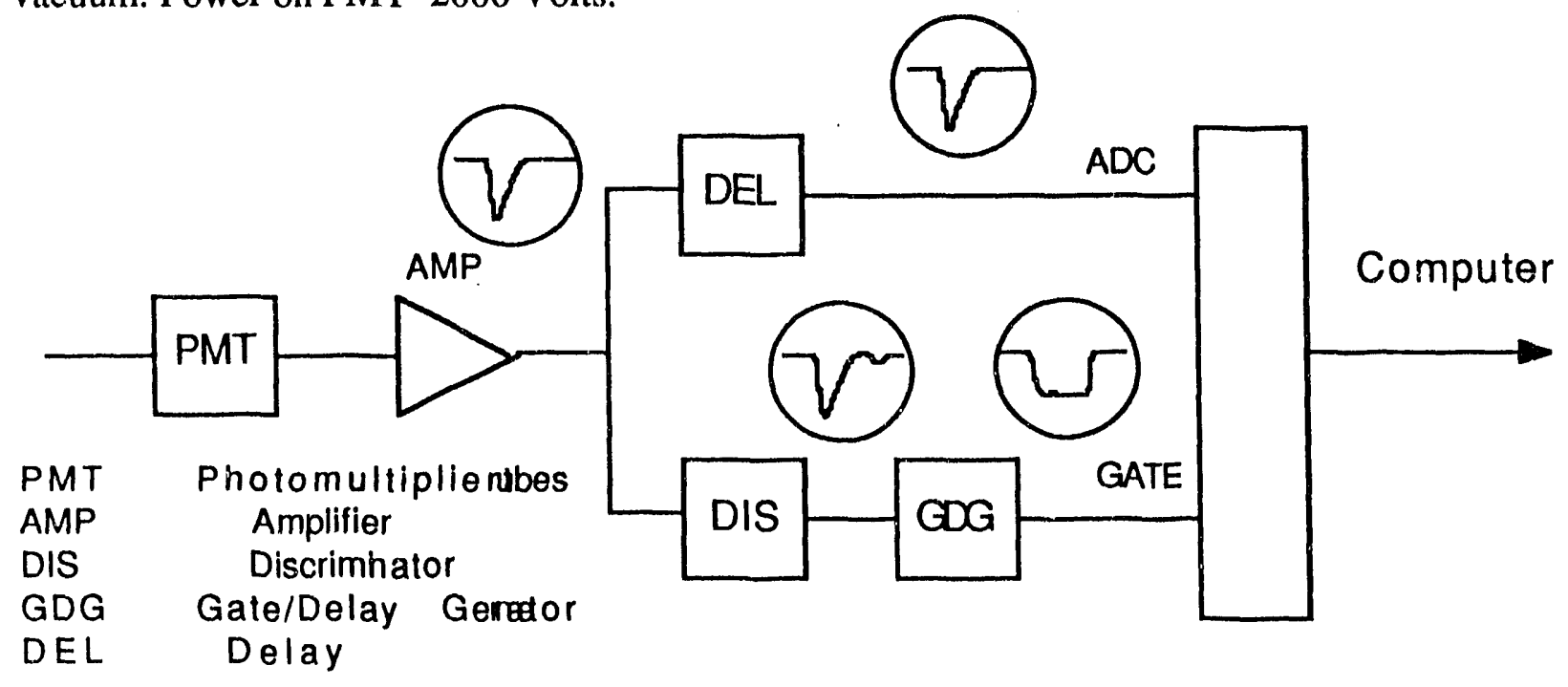

Figure 13. Electric configuration and signals.

As we see in figure 13 the pulse from the PMT is amplified and then goes into two branches. In the upper one the pulse is just delayed. In the lower one the amplified pulse goes into a discriminator and trigs it at a certain pulse height, which we can chose. This is done because we do not want it to trig a gate pulse on the noise. So when there is a gate pulse a conversion is done in the ADC. The digital number then goes into the computer and a histogram is made.

FIGURE HISTOGRAM (PMT-1 DARK, PMT-1 LIGHT AND MAYBE OVERLAYED.) 


\title{
THE SECOND MEASUREMENT.
}

The same test fixture as in the first experiment. System pumped down to pre-vacuum and a radioactive Californium 252 source mounted in the chamber. A one layer plastic film is inserted in the chamber. Power supply PMT -1800 Volts. Runtime 3 hours 45 minutes.

\section{FIGURE HISTOGRAM PMT-1 CF-TEST.1}

Unfortunally we can not see any fission fragments in this histogram. The radioactive source is rather old and probably weak. There is a lot of counts on the left hand side which probably is alfa particles and noise in the PMT. To get rid of more of the noise the threshold is moved higher up on the peak which hopefully is going to give us a better histogram.

\section{THE THIRD MEASUREMENT.}

Same as privies experiment but we now trig the discriminator higher on the amplified pulse from PMT. Runtime 2 hours and 30 minutes.

\section{FIGURE HISTOGRAM PMT-1 CF-TEST.2}

This histogram shows us that we have fission products. We can see to peaks.

\author{
VIII- FUTURE TESTS.
}

IX- CONCLUTIONS. 


\section{Appendix C}

\section{LAST Detector Engineering Drawings}




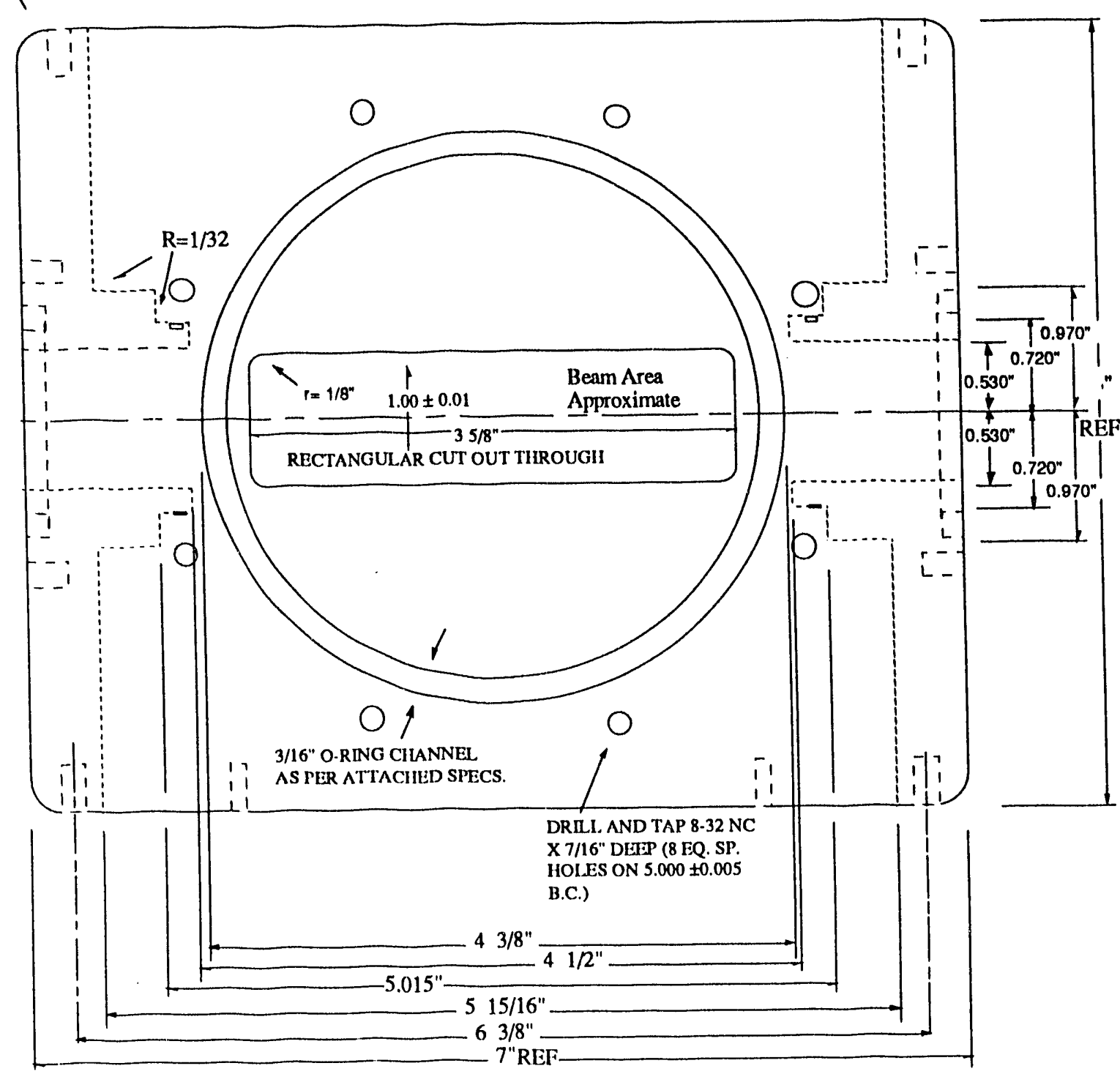
SPACE ASTRONOMY LABORATORY
University of Florida

Project: LAST Detector

Title: Det. Vacuum Chamber Quantity: 2

Material: $6061-\mathrm{T} 6 \mathrm{Al}$

Drawn By: D. Ely

Date: 1 Jun 89

Tolerance: $\pm 0.005^{*}$ dec., $\pm 1 / 32^{*}$ lract. 


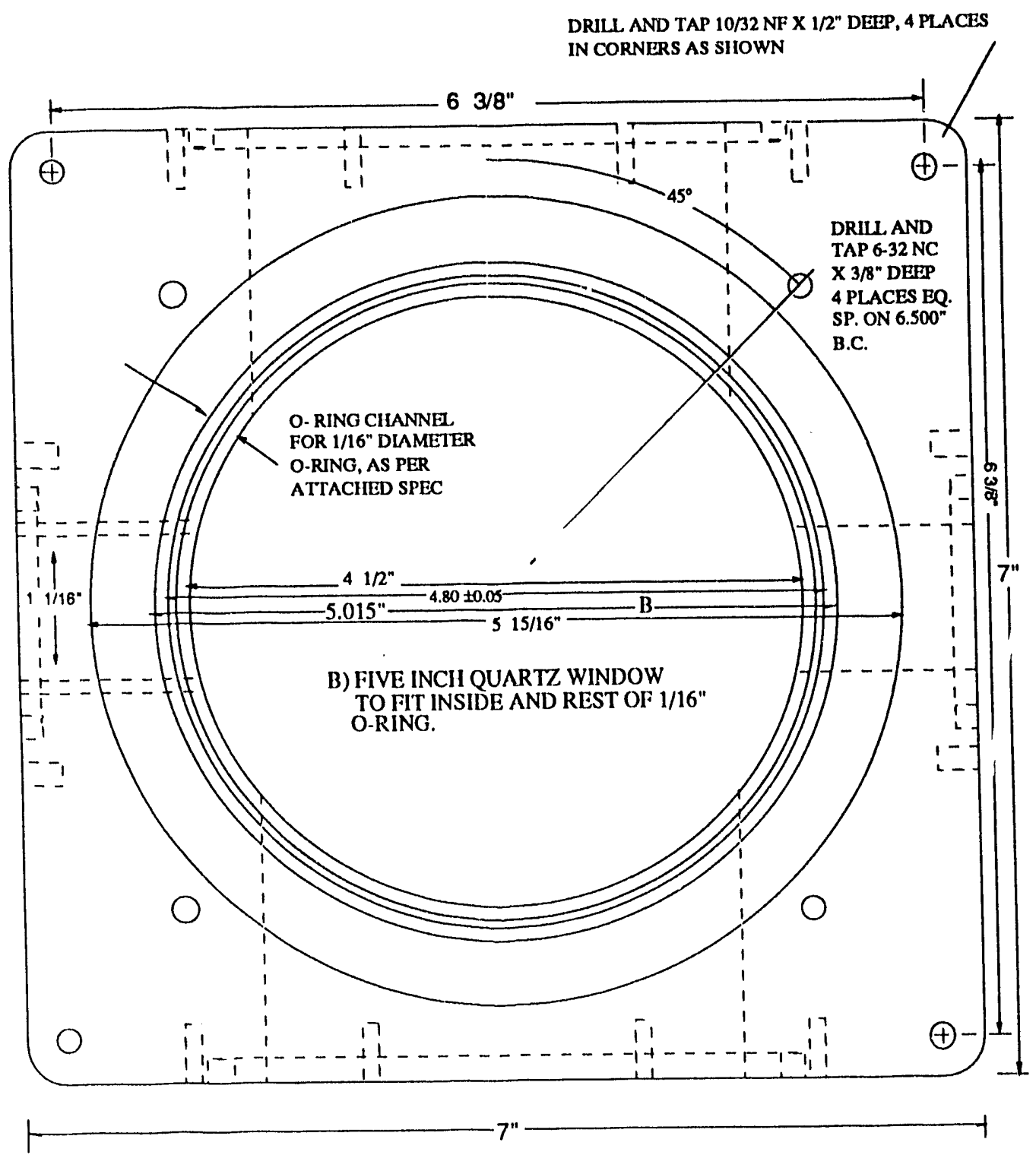

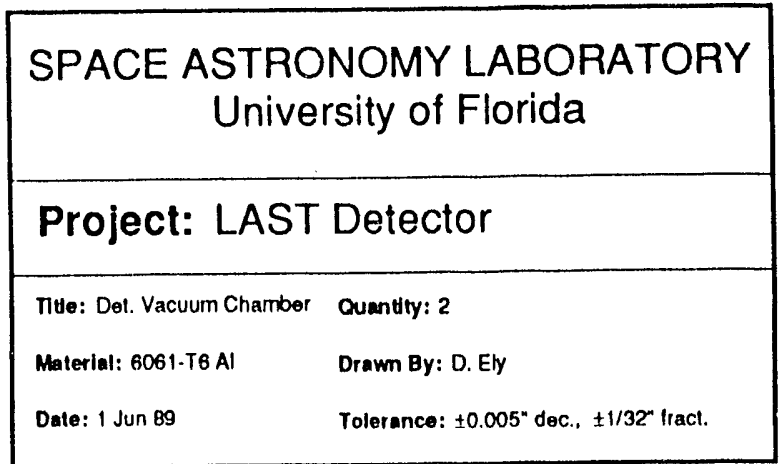



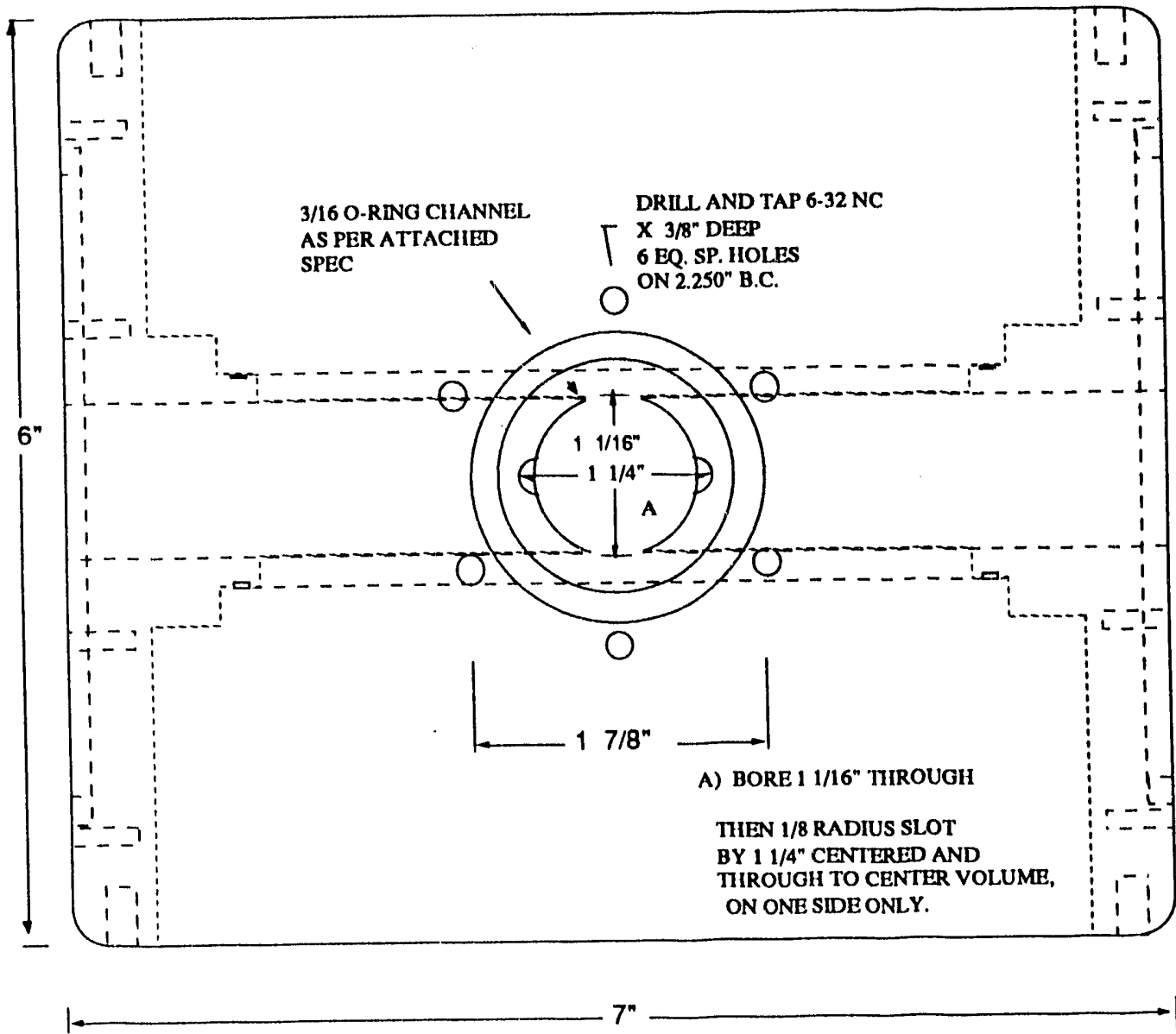

$7^{n} \longrightarrow$

\section{SPACE ASTRONOMY LABORATORY} University of Florida

Project: LAST Detector

Tlue: Det. Vacuum Chamber Quantity: 2

Materlal: 6061-T6 Al

Drawn By: D. Ely

Dale: 1 Jun 89

Tolerance: $\pm 0.005^{n}$ dec., $\pm 1 / 32^{*}$ fract. 

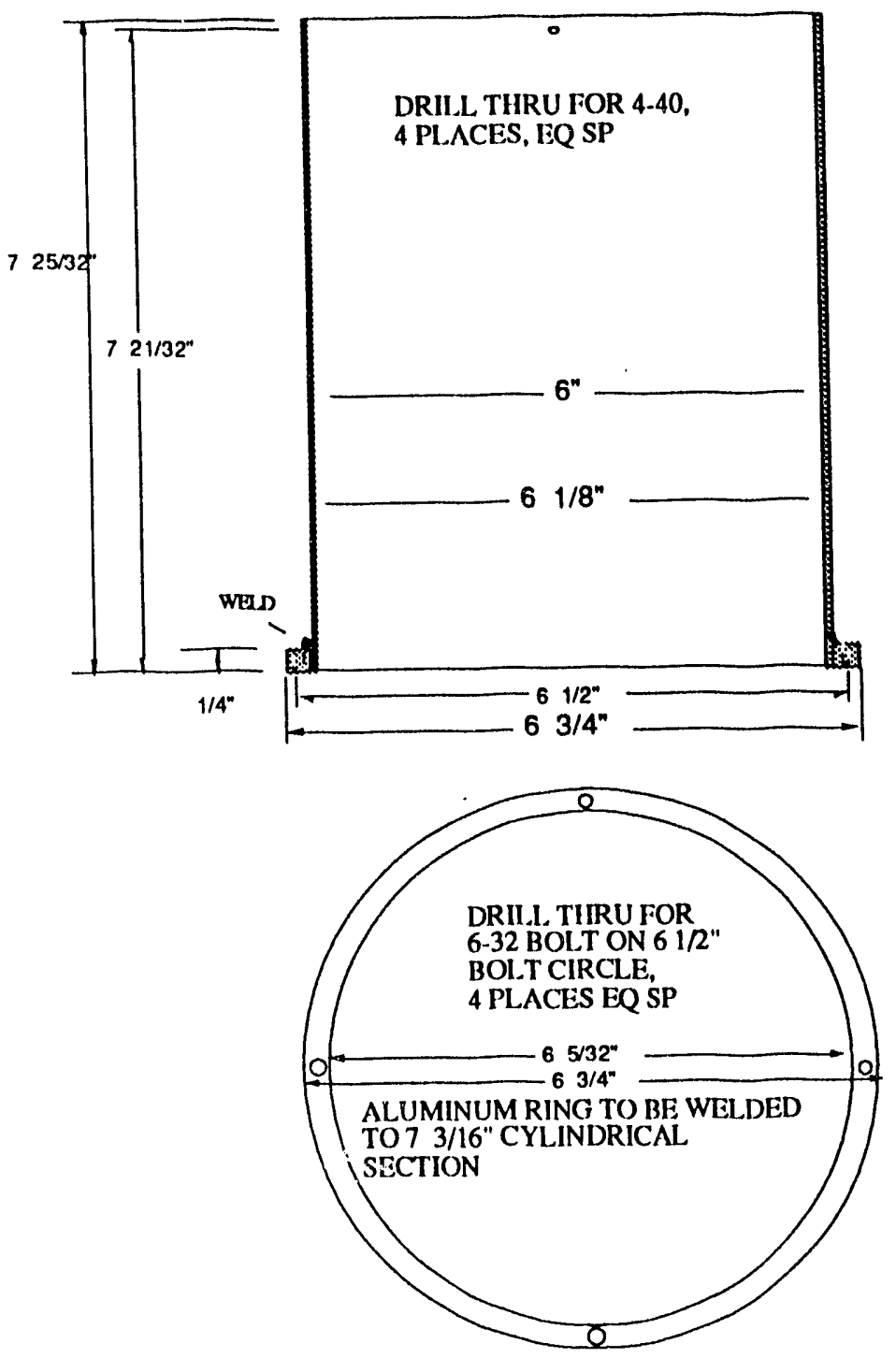

$1 / 4^{\prime \prime}$

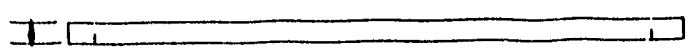

\section{SPACE ASTRONOMY LABORATORY University of Florida}

Project: LAST Detector

Title: Lower PM T Housing

Material: Aluminum

Date: 27 Apt 89
Cuantity: 2

Drawn By: D. Ely

Tolerance: $\pm 0.005^{\circ}$ dec., $\pm 1 / 32^{\prime \prime}$ fract. 


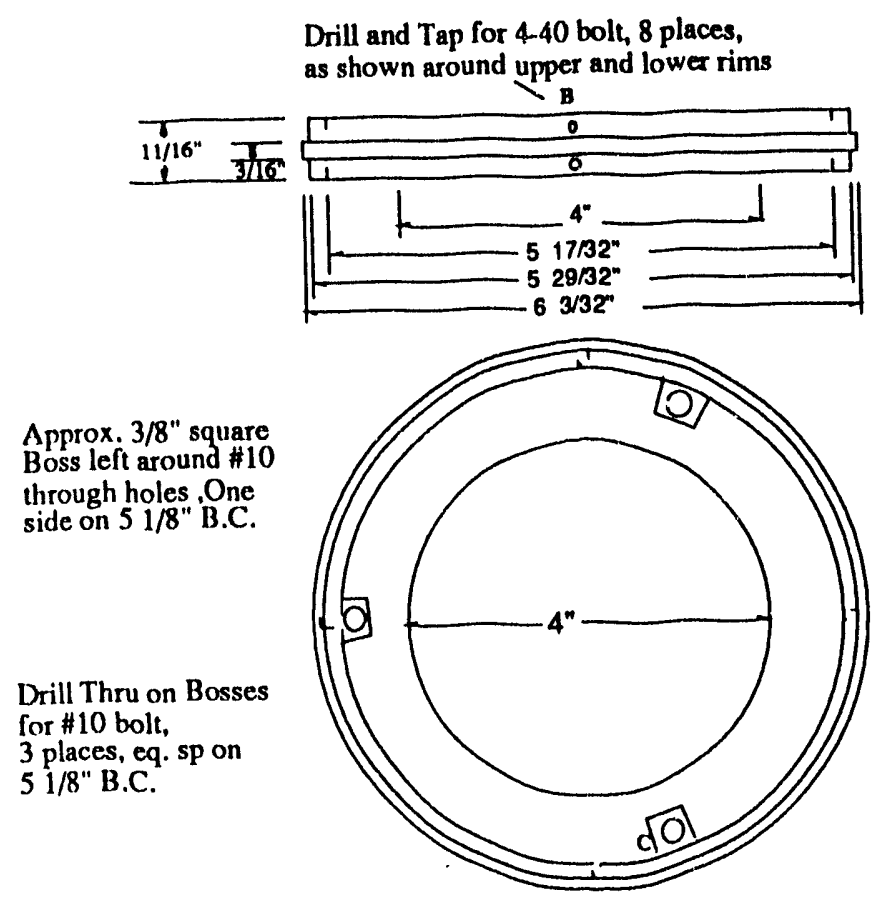

SPACE ASTRONOMY LABORATORY University of Florida

Project: LAST Detector

TIse: РMT Cpaluro Interface Ouantity: 2

Material: Aluminum Drawn By: D. Ely

Date: 27 Apr $89 \quad$ Tolerance: $\pm 0.005^{\prime \prime}$ dec., $\pm 1 / 32^{\prime \prime}$ fract. 
Drill and Tap deep enough to seat

\#10-24 threaded rod(1 3/8")

3 places, eq sp on $51 / 8$ " BC

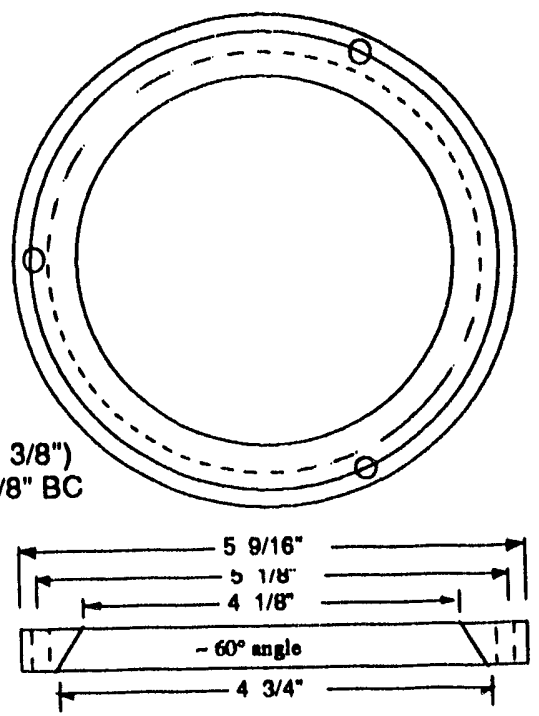

SPACE ASTRONOMY LABORATORY University of Florida

Project: LAST Detector

Nile: PMT Capture Ring Quantity: 2

Maverlat: Phenolk Drawn By: D. Ely

Date: $27 \mathrm{Apr} 89$ Tolerance: $\pm 0.005^{n}$ dec., $\pm 1 / 32^{n}$ tract. 


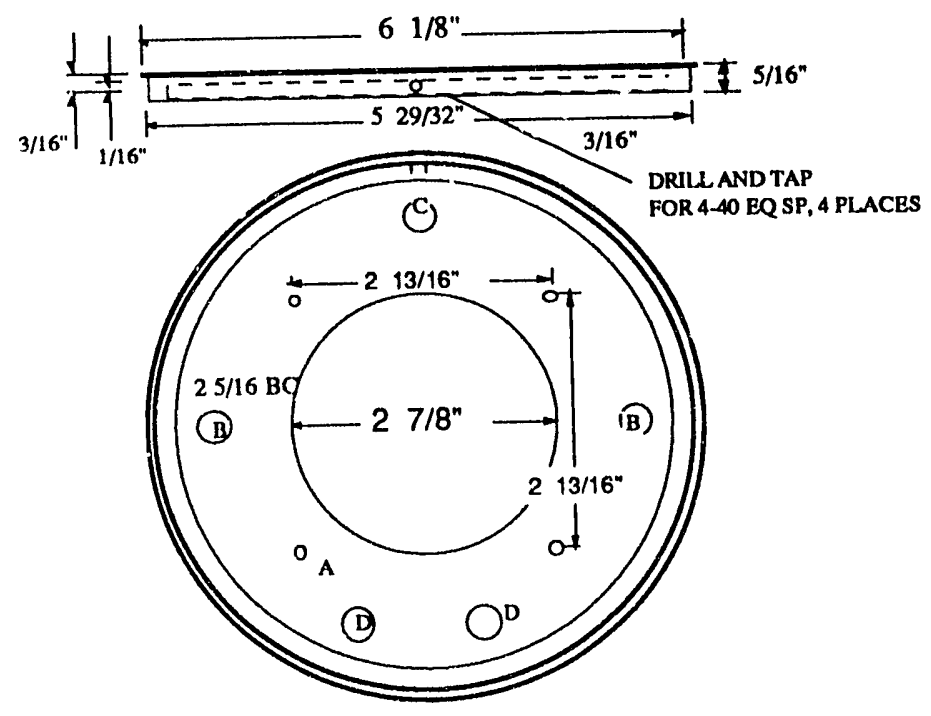

A) DRILL THRU FOR *4, FOUR PLACES AS SHOWN

B) 0.625 DRILL THROUGH TWO PLACES AS SHOWN ON 4 5/8" BOLT CIRCLE

C) 0.500 DRILL TIIROUGH ONE PLACE AS SHOWN ON 4 5/8" B.C.

D) 0.375 DRILL TIIROUGH TWO PLACES AS SHWON ON 4 5/8" B.C.

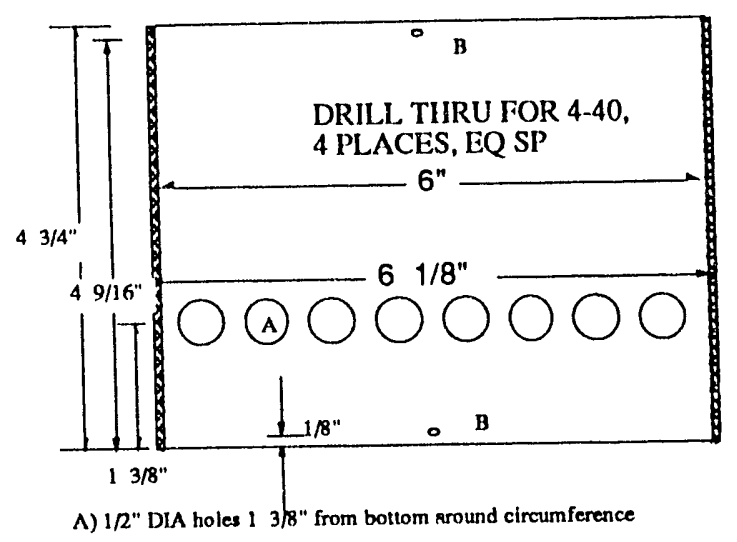

B) DRILL, THRU FOR 4-40, 4 PLACES, EQ SP

\begin{tabular}{|c|c|}
\hline \multicolumn{2}{|c|}{ Project: LAST Detector } \\
\hline THe: Base Top and Cover & Ouanuly: 2 Each \\
\hline Material: Aluminum & Drewn By: D. Ely \\
\hline Date: $22 \mathrm{Dec} 88$ & Tolerancs: \pm 0.005 " doc., $\pm 1 / 32$ " lract. \\
\hline
\end{tabular}




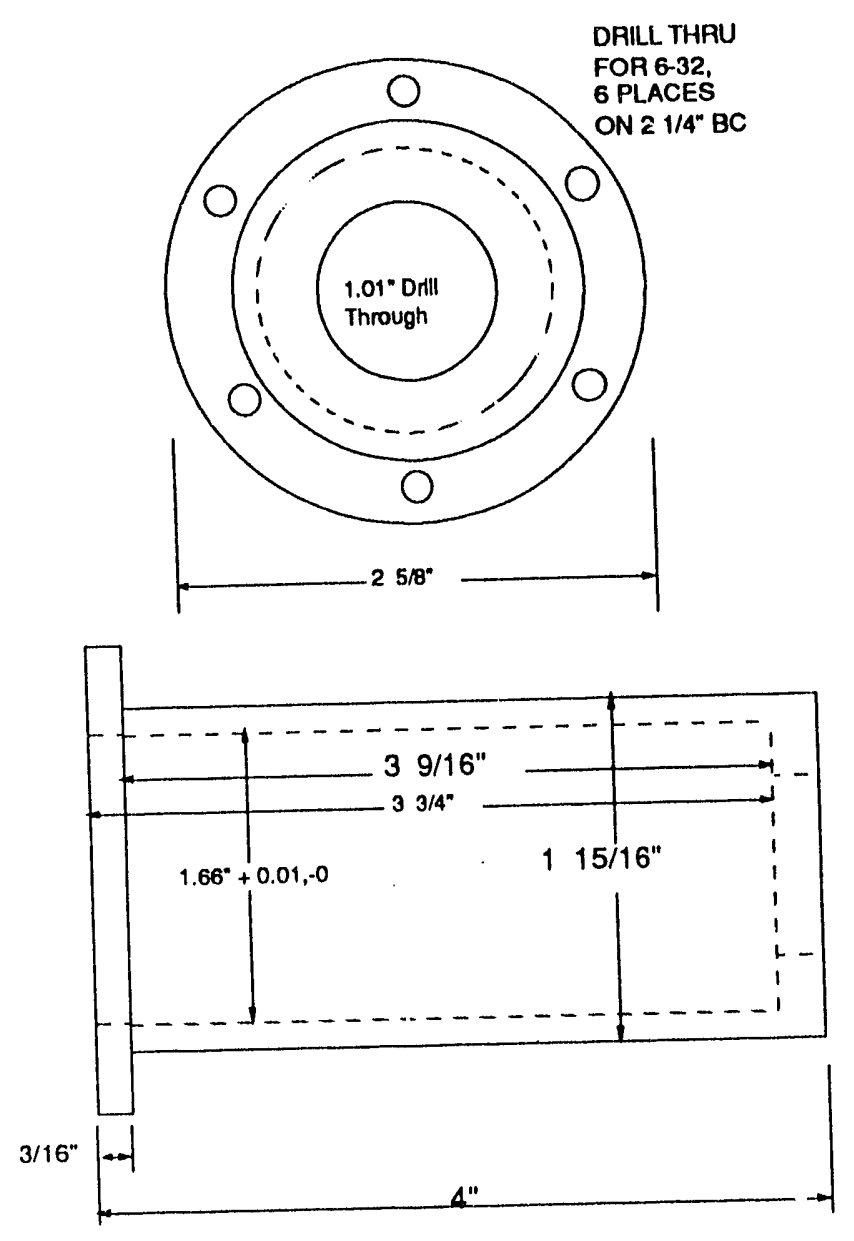

\begin{tabular}{|c|c|}
\hline \multicolumn{2}{|c|}{$\begin{array}{c}\text { SPACE ASTRONOMY LABORATORY } \\
\text { University of Florida }\end{array}$} \\
\hline \multicolumn{2}{|c|}{ Project: LAST Detector } \\
\hline The: Feedihrougn Adapler & Quantity: 1 \\
\hline Material: Alumlnum & Drawn By: D. Ely \\
\hline Dale: 27 Apr 89 & Tolerance: $\pm 0.005^{*}$ dec., $\pm 1 / 32^{*}$ Iract. \\
\hline
\end{tabular}




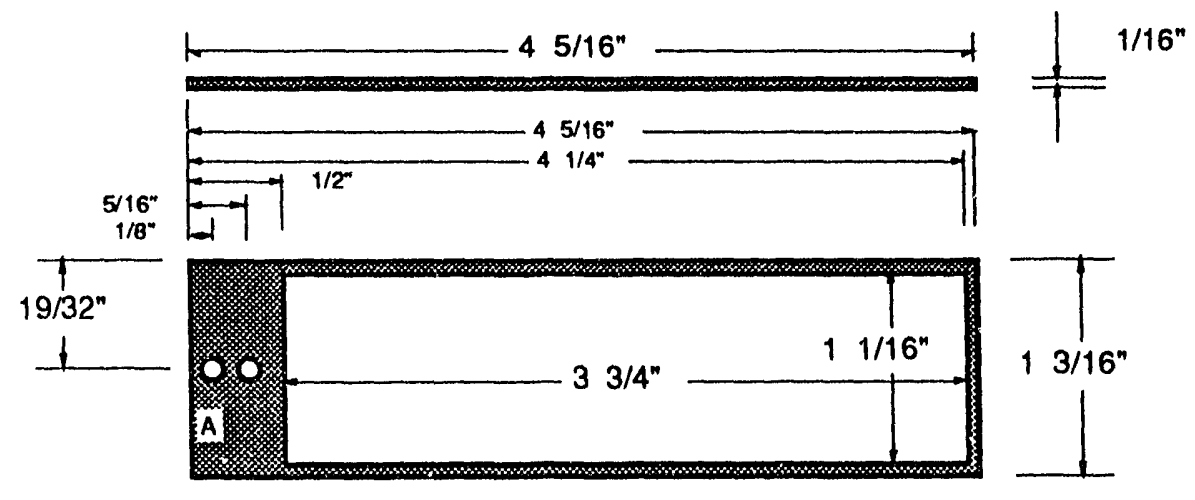

A) DRILL THROUGH FOA \#2 SCREW

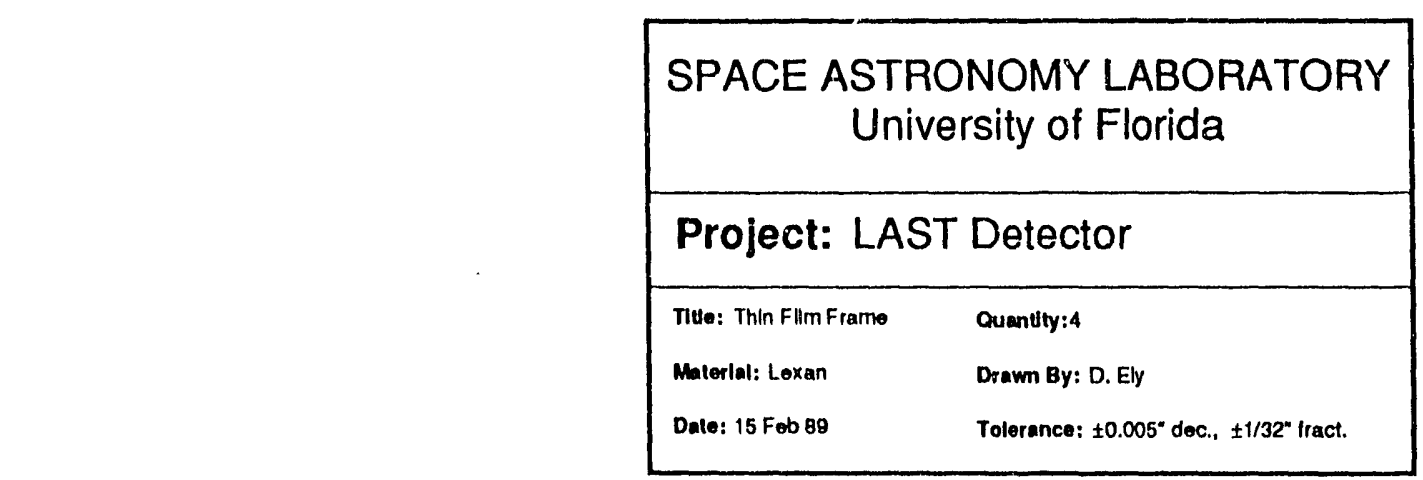

\#51 Drill Thru, 2 places

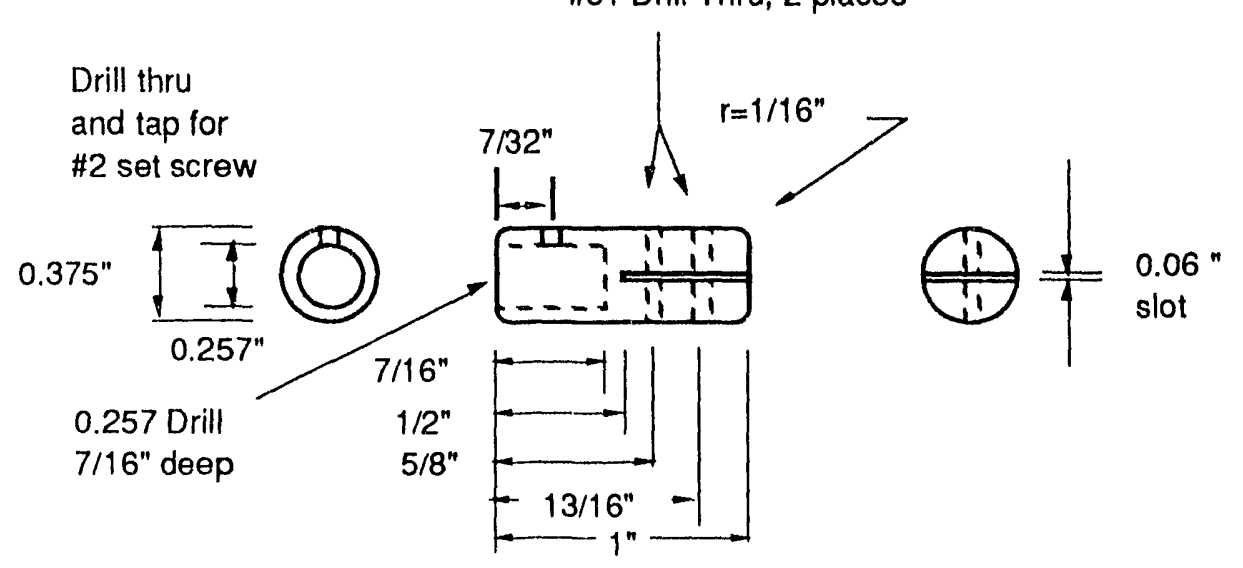

\begin{tabular}{|c|c|}
\hline \multicolumn{2}{|c|}{$\begin{array}{c}\text { SPACE ASTRONOMY LABORATORY } \\
\text { University of Florida }\end{array}$} \\
\hline \multicolumn{2}{|c|}{ Project: LAST Detector } \\
\hline Tiue: Frame to Rou Adaplor & Quantity: 1 \\
\hline Malerian: Aluminum & Drawn By: D. Ely \\
\hline Dale: 17 Nolv 88 & Tolerance: $\pm 0.005^{\circ}$ doc., $\pm 1 / 32^{\prime \prime ~ f r a c t . ~}$ \\
\hline
\end{tabular}




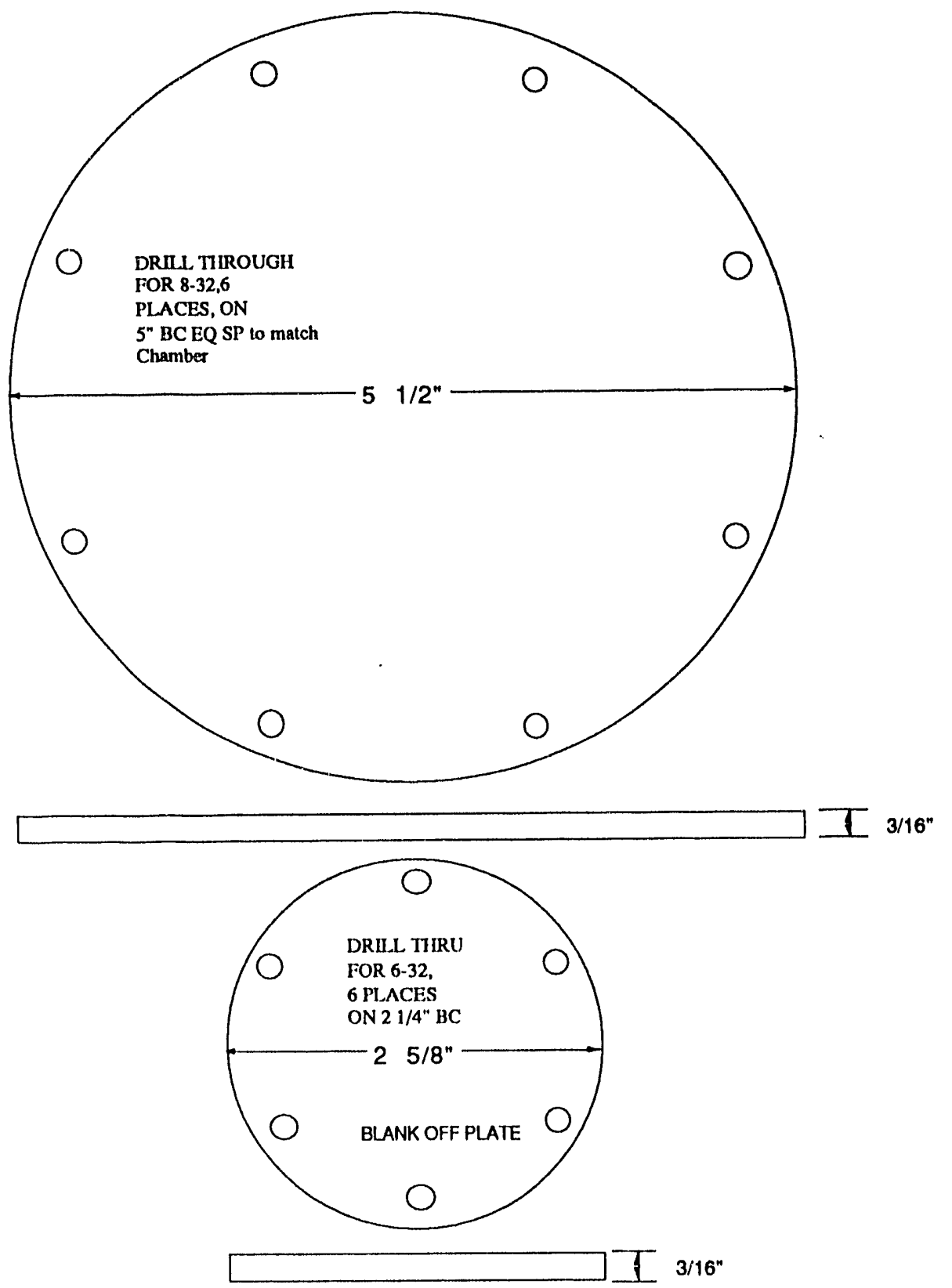

SPACE ASTRONOMY LABORATORY University of Florida

Project: LAST Detector

TIte: Blank Otf Plates

Material: Aluminum

Date: 1 Nov 88
Quantity: 1 Each

Drawn By: D. Ely

Tolerance: $\pm 0.005^{\prime \prime}$ dec., $\pm 1 / 32^{\prime \prime}$ fract. 


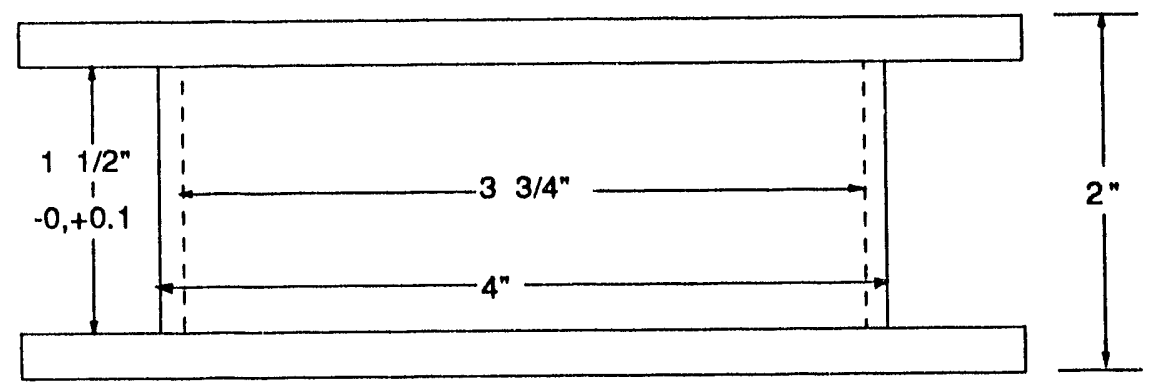

DRILL TIIROUGH
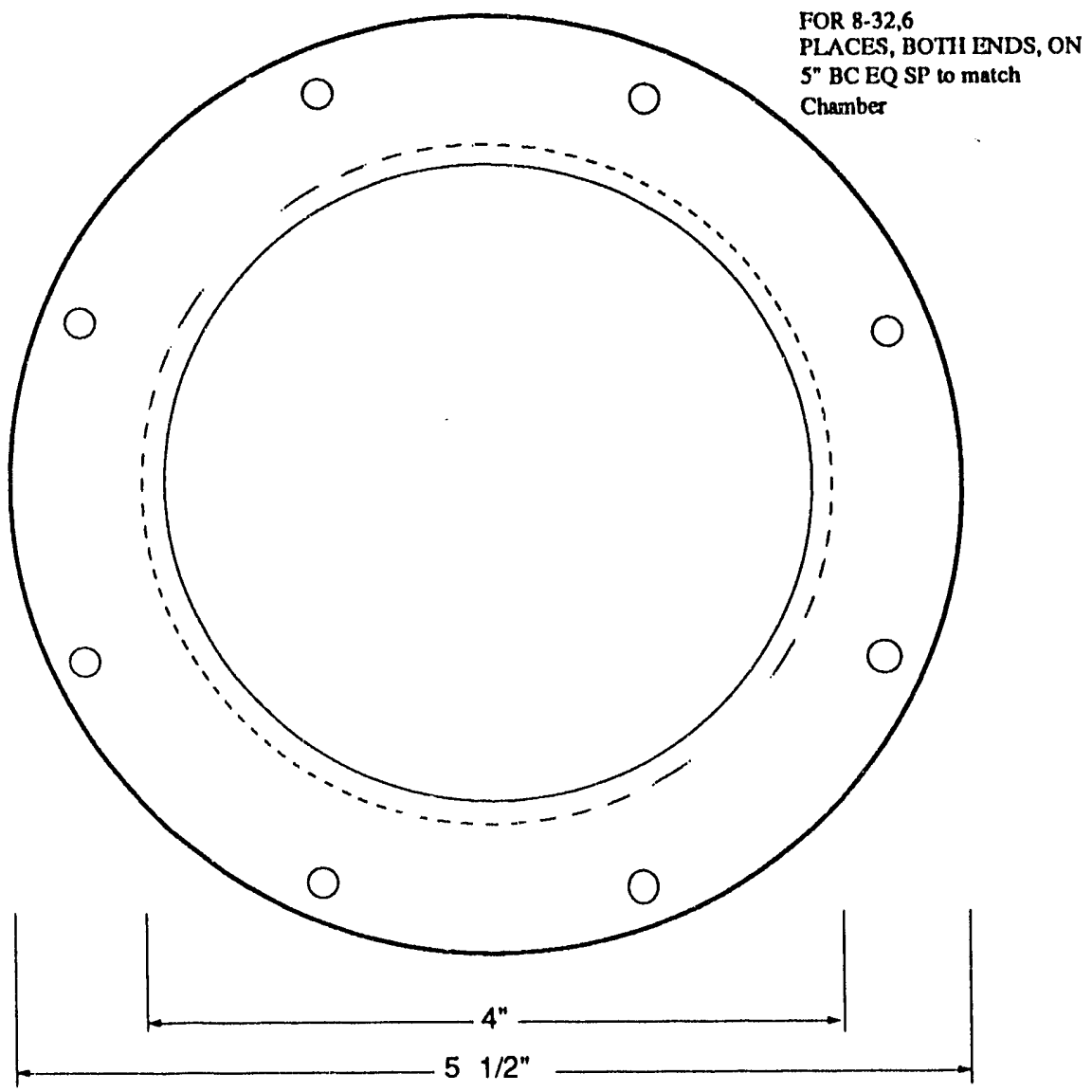

SPACE ASTRONOMY LABORATORY University of Florida

Project: LAST Detector

nite: Chamber to Chamb

Material: Aluminum

Date: $10 \mathrm{Apr} 89$
Cuanisty: 1

Drawn By: D. Ely

Tolerance: $\pm 0.005^{\prime \prime}$ dec., $\pm 1 / 32 "$ fract 

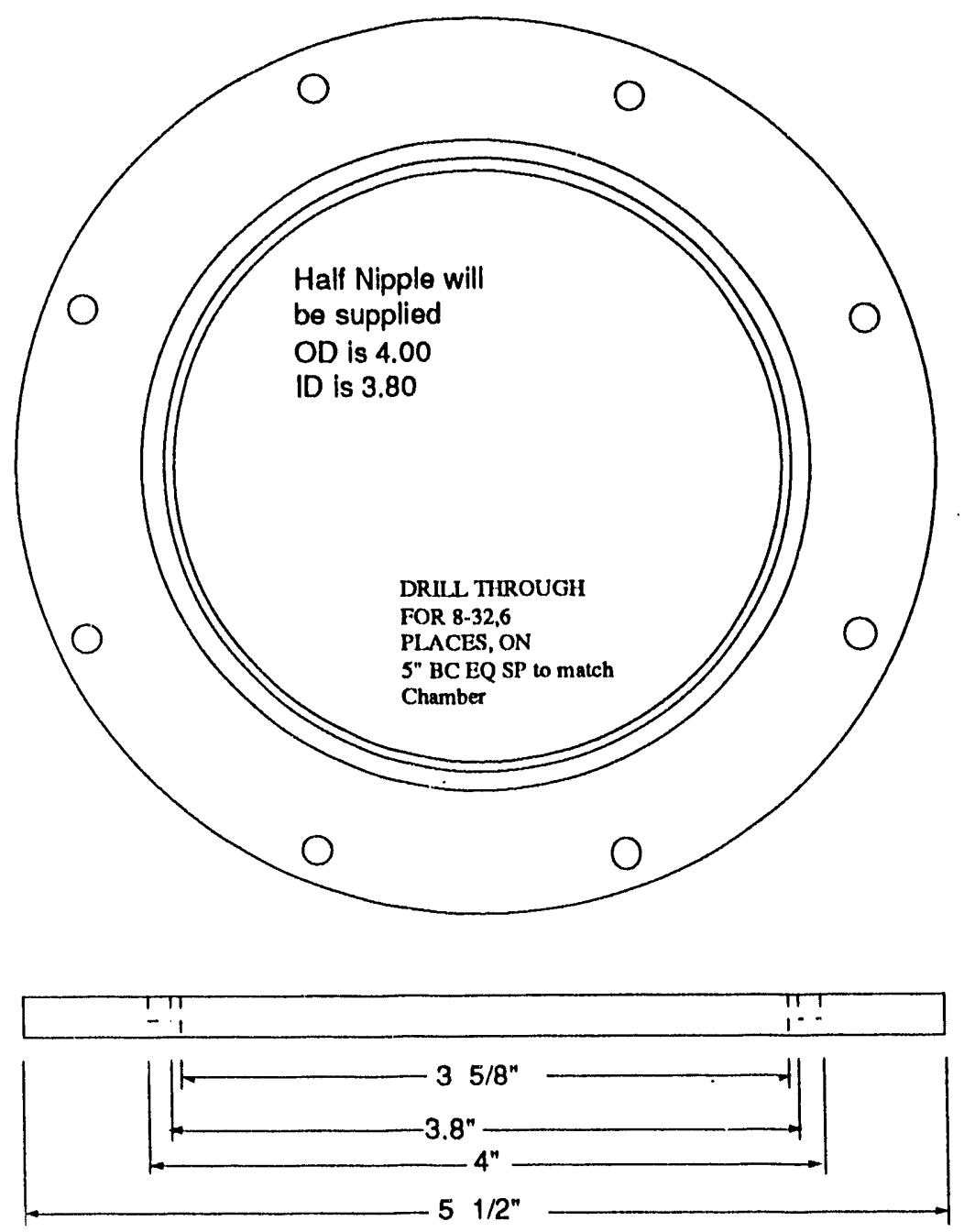

\begin{tabular}{|c|c|}
\hline \multicolumn{2}{|c|}{$\begin{array}{l}\text { SPACE ASTRONOMY LABORATORY } \\
\text { University of Florida }\end{array}$} \\
\hline \multicolumn{2}{|c|}{ Project: LAST Detector } \\
\hline Tile: Flange Adapter & Quantity: 1 \\
\hline Materlal: Stainless Sleel & Drawn By: D. Ely \\
\hline Date: 24 Oct 88 & Tolerance: $\pm 0.005 "$ dec., $\pm 1 / 32^{\prime \prime ~ f r a c t . ~}$ \\
\hline
\end{tabular}



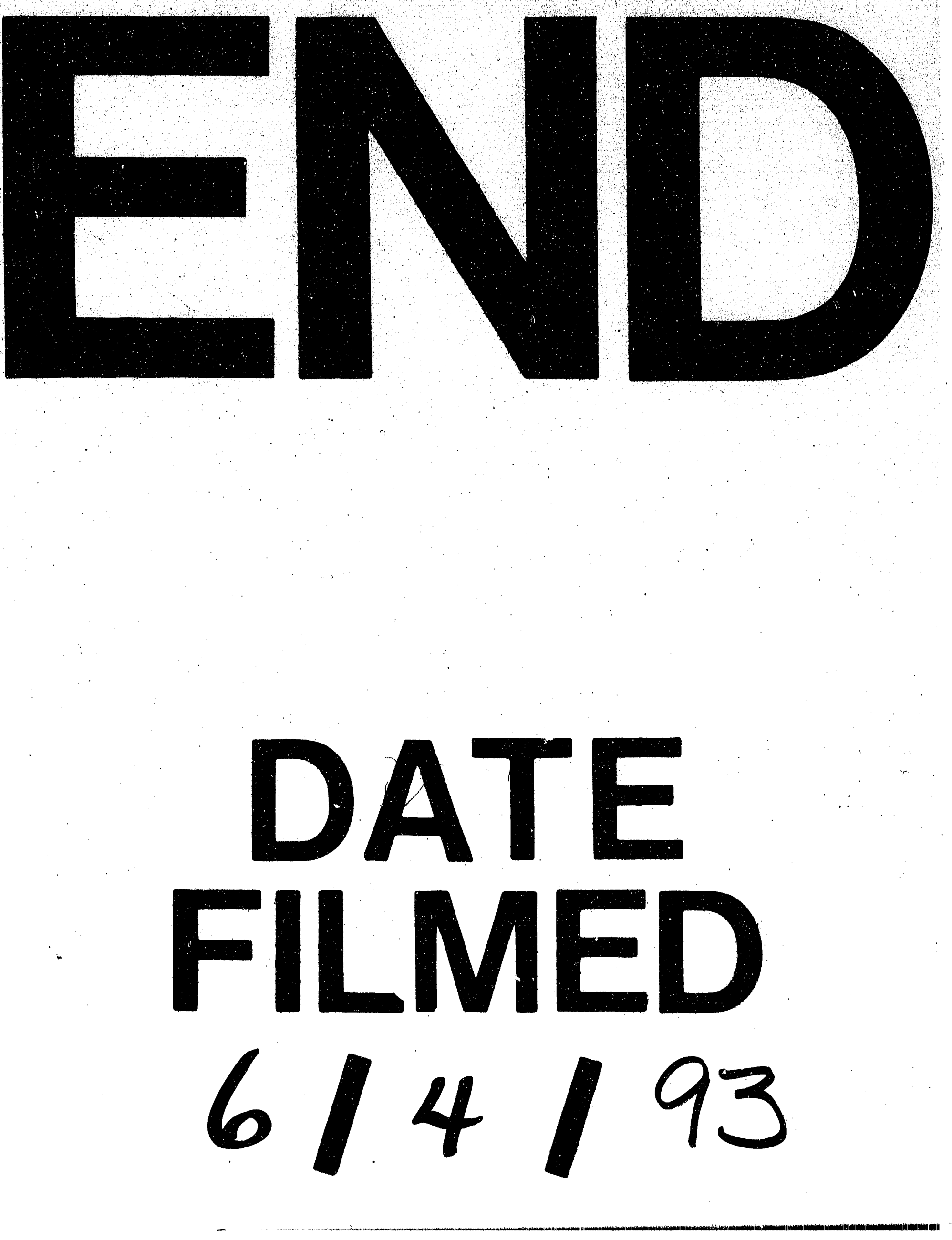
